\title{
ANDRÉ KONDO
}

\section{Abordagem endoscópica comparada à cirúrgica no tratamento do câncer gástrico precoce: revisão sistemática e metanálises}

Tese apresentada à Faculdade de Medicina da Universidade de São Paulo para obtenção do título de Doutor em Ciências

Programa de Ciências em Gastroenterologia Orientador: Prof. Dr. Eduardo Guimarães Hourneaux de Moura 
Dados Internacionais de Catalogação na Publicação (CIP)

Preparada pela Biblioteca da

Faculdade de Medicina da Universidade de São Paulo

Creprodução autorizada pelo autor

Kondo, André

Abordagem endoscópica comparada à cirúrgica no tratamento do câncer gástrico precoce: revisão sistemática e metanálises / André Kondo. -- São Paulo, 2016.

Tese(doutorado)--Faculdade de Medicina da Universidade de São Paulo.

Programa Ciências em Gastroenterologia.

Orientador: Eduardo Guimarães Hourneaux de Moura.

Descritores: 1.Estômago 2.Neoplasias gástricas 3.Endoscopia 4.Endoscopia do sistema digestório 5.Endoscopia gastrointestinal 6.Gastroscopia 7.Gastrectomia 8.Procedimentos cirúrgicos operatórios 9.Procedimentos cirúrgicos do sistema digestório 10.Revisão sistemática 11.Metanálise

USP/FM/DBD-336/16 
"Determinação, coragem e autoconfiança são fatores decisivos para o sucesso. Não importa quais sejam os obstáculos e as dificuldades. Se estamos possuídos de uma inabalável determinação, conseguiremos superá-los. Independentemente das circunstâncias, devemos ser sempre humildes, recatados e despidos de orgulho". 
Aos meus avós Rikiti e Yoshie, Keinosuke e Suye, que sempre me incentivaram e me ensinaram a nunca parar de estudar.

Aos meus pais, Akira e Sueli, responsáveis por me educar nos princípios e valores mais importantes da vida.

Ao meu irmão, Felipe, fiel amigo e autêntico companheiro.

À minha amada esposa, Gabriella, e aos nossos maravilhosos filhos, Dudu e Rafa, minhas maiores e estimadas riquezas, motivos para eu querer viver. 


\section{AGRADECIMENTOS}

Aos Professores Ivan Cecconello e Luiz Augusto Carneiro D'Albuquerque, pela coordenação do Departamento de Gastroenterologia da FMUSP nas Disciplinas de Cirurgia do Aparelho Digestivo, Coloproctologia e Transplante de Órgãos do Aparelho Digestivo, assegurando condições para o fortalecimento da pesquisa com qualidade e relevância.

Ao Professor Paulo Sakai, renomado endoscopista, sempre presente e exemplo de incansável motivação.

Ao Professor Eduardo Guimarães Hourneaux de Moura, pela orientação científica, oportunidade profissional e amizade criada nestes últimos anos.

Ao Professor Wanderley Marques Bernardo, pelos ensinamentos, conselhos e auxílio na confecção deste trabalho.

Ao Professor Osmar Kenji Yagi, grande amigo, pela colaboração na elaboração desta Tese.

Aos Professores Angelita Habr-Gama, Joaquim Gama-Rodrigues e Bruno Zilberstein, por me fazerem enxergar a cirurgia com encanto e deslumbramento.

Aos colegas e amigos do Grupo de Cirurgia do Estômago e Intestino Delgado, ou que dele fizeram parte, André Roncon Dias, Carlos Eduardo Jacob, Cláudio José Caldas Bresciani, Cláudio Roberto Deutsch, Donato Roberto Mucerino, Fábio Pinatel Lopasso, Marcelo Mester, Marcus Kodama P. Ramos, Mitsunori Matsuda, Paulo César Leonardi e Ulysses RibeiroJúnior, pelos anos de experiência. 
Aos grandes amigos André de Souza Potenza, Nivaldo Marques Cabral, Raquel Ajub Moysés e Ronaldo Elias Carnut Rego, por contribuírem com minha formação médica e pessoal.

Aos Doutores Antônio Carlos Pereira Lima e Renato Arioni Lupinacci, pelo espaço proveitoso no meu início de carreira e pela possibilidade de capacitação profissional.

Aos assistentes do Serviço de Endoscopia Gastrointestinal do HCFMUSP, Adriana Vaz Safatle-Ribeiro, Carlos Kiyoshi Furuya Júnior, Christiano Makoto Sakai, Caterina Maria Pia Simioni Pennacchi, Dalton Marques Chaves, Edson Ide, Elisa Ryoka Baba, Eunice Komo Chiba, Everson Luiz de Almeida Artifon, Gustavo de Oliveira Luz, Jeane Martins Melo, Kendi Yamazaki, Kengo Toma, Luiz Henrique Mazzonetto Mestieri, Marcelo Magno de Freitas Sousa, Marcos Eduardo Lera dos Santos, Nelson Tomio Miyajima, Renato Baracat, Róbson Kiyoshi Ishida, Rogério Kuga, Sebastião Alves Dantonio, Sérgio Barbosa Marques, Sérgio Eiji Matuguma, Sérgio Shiguetoshi Ueda, Sônia Nádia Fylyk, Spencer Cheng, Thiago Ferreira de Souza, Tomazo Antônio Prince Franzini e Toshiro Tomishige, pelos ensinamentos e companhia profícua.

Aos colegas e amigos de Residência Médica em Cirurgia do Aparelho Digestivo, Allan Garms Marson, Antônio Couceiro Lopes, Arceu Scanavini Neto, Bruno da Costa Martins, Caio Sérgio Rizkallah Nahas, Carlos Eduardo Fonseca Pires, César Giovani Conte, Eduardo Freitas Viana, Émerson Shigueaki Abe, Fábio Crescentini, Fabrício Ferreira Coelho, Francisco Carlos Bernal da Costa Seguro, Guilherme Cutait de Castro Cotti, José Américo Bacchi Hora, Luciana Bertocco de Paiva Haddad, Marcelo Simas de Lima, Nélio Reis Ferreira, Paulo Jacques Gherardi Goldstein, Renato Micelli Lupinacci e Rodrigo Ambar Pinto, pelos anos de agradável e inesquecível convívio. 
Aos colegas e amigos de Residência Médica em Endoscopia Gastrointestinal do HCFMUSP, Ana Carolina Strake Navarro, Benilton Batista de Souza, Carolina Eliane Reina Forster, Cíntia Morais Lima dos Santos, Débora da Costa Vieira Albers, Diogo Turiani Hourneaux de Moura, Eduardo Turiani Hourneaux de Moura, Ernesto Quaresma Mendonça, Fábio Alberto Castillo Bustamante, Felipe Iankelevich Baracat, Flávio Hiroshi Ananias Morita, Hélcio Cardoso Gomes, Joel Fernandez de Oliveira, José Gonçalves Pereira Bravo, Júlio César Martins Aquino, Leonardo Zorrón Cheng Tao Pu, Mariana lennaco de Siqueira Campos, Marianny Nazareth Sulbaran Nava, Marina Lordello Passos, Maurício Kazuyoshi Minata, Mileine Valente de Matos, Murilo Folharini Catalano, Nádia Korkischko, Priscilla Cavalheiro Bonifácio, Renata Nobre Moura, Rodrigo Silva de Paula Rocha, Silvia Lúcia Alves de Lima e Vinicius Leite de Castro, pelo apoio e grandes momentos em minha trajetória profissional.

Ao amigo Gustavo Luís Rodela Silva, pelo apoio durante minha formação e pelos primorosos desenhos desta Tese.

À senhora Marta Regina Rodrigues, pela constante e infindável disposição para ajudar.

À senhora Maria Helena Vargas, pela revisão e diagramação desta Tese, assim como pela pronta ajuda e disponibilidade em todos os momentos.

A Ana Carolina Cândido, Bruna Freitas, Fabiana Renata Soares Bispo, Fernanda Masiero, Marcos Retzer (in memoriam), Maria do Carmo da Silva, Maria Joelice dos Reis Santos, Maria Cristina Rabelo, Marisa Conceição Morais, Marisa Ochner, Myrtes Freire de Lima, Paula Zoubaref, Roberto Martins, Suzidarley Guimarães Rodrigues e Vilma de Jesus Libério, pela assistência em todos os momentos.

Aos pacientes e a todos os profissionais, dos diversos Institutos do complexo HC-FMUSP que, direta ou indiretamente, contribuíram para a realização deste estudo e minha formação profissional, como cirurgião e endoscopista. 
Esta tese está de acordo com as seguintes normas, em vigor no momento desta publicação:

Referências: adaptado de International Committee of Medical Journals Editors (Vancouver).

Universidade de São Paulo. Faculdade de Medicina. Serviço de Biblioteca e Documentação. Guia de apresentação de dissertações, teses e monografias.

Elaborado por Anneliese Carneiro da Cunha, Maria Julia de A. L. Freddi, Maria F. Crestana, Marinalva de Souza Aragão, Suely Campos Cardoso, Valéria Vilhena. $3^{a}$ ed. São Paulo: Divisão de Biblioteca e Documentações; 2011.

Abreviatura dos títulos dos periódicos de acordo com List of Journals Indexed in Index Medicus. 


\section{SUMÁRIO}

Lista de abreviaturas e siglas

Lista de figuras

Lista de gráficos

Resumo

Abstract

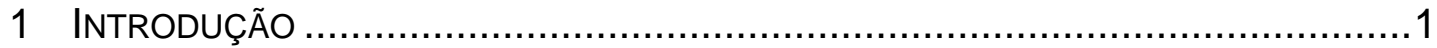

1.1 Mucosectomia e Dissecção Endoscópica Submucosa........................14

1.2 Outras Técnicas de Tratamento Endoscópico.................................17

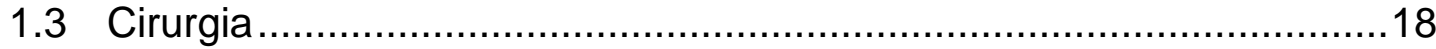

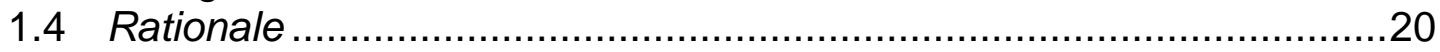

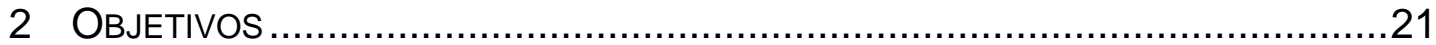

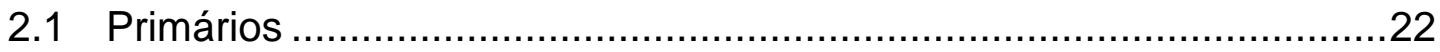

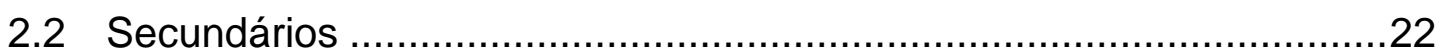

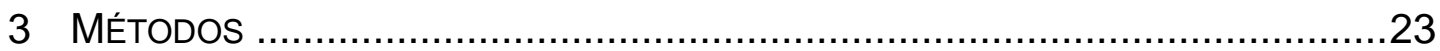

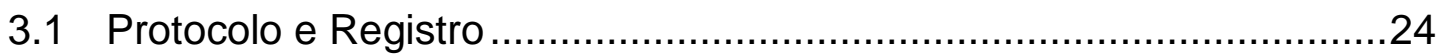

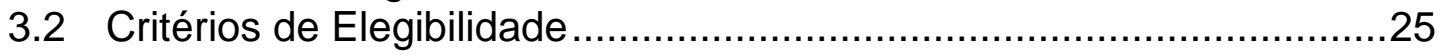

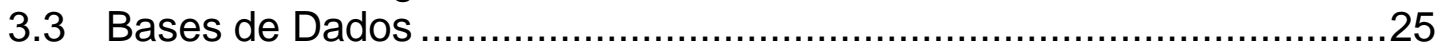

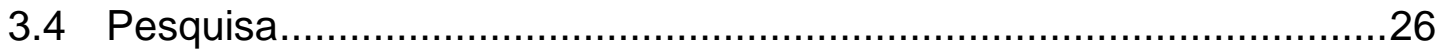

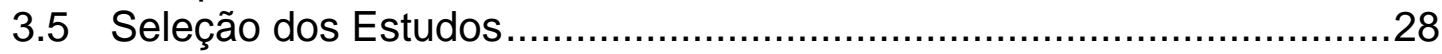

3.6 Processo de Coleta de Dados .................................................... 28

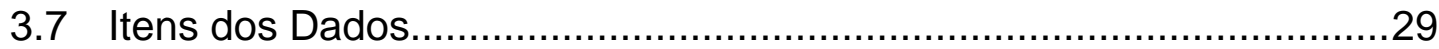

3.8 Riscos de Vieses nos Estudos Individuais .......................................30

3.9 Medidas de Síntese e Análise ..................................................... 31

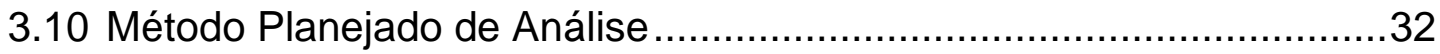

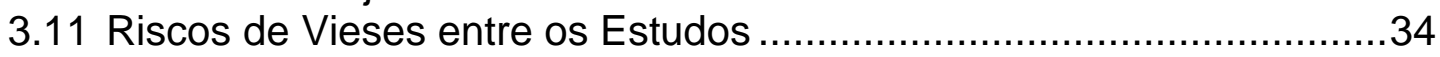

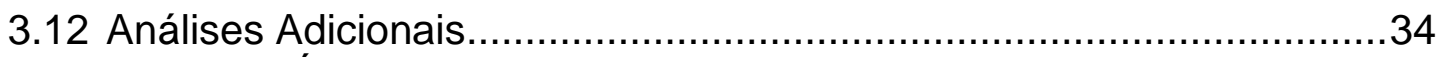

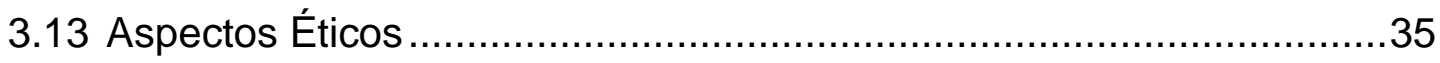

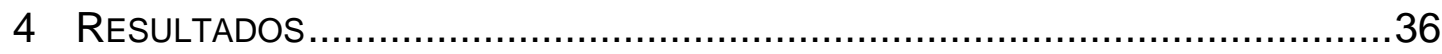

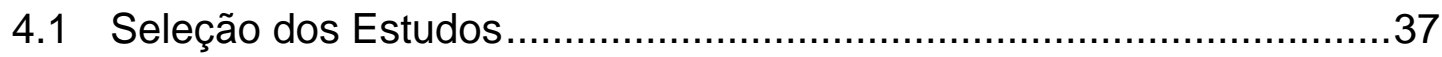

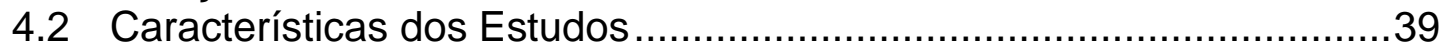

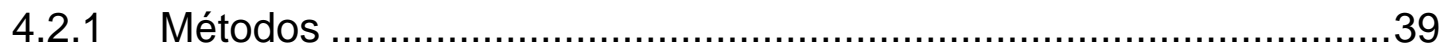

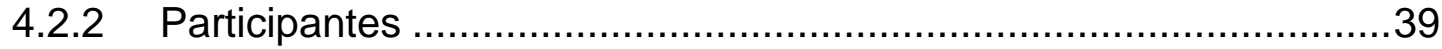

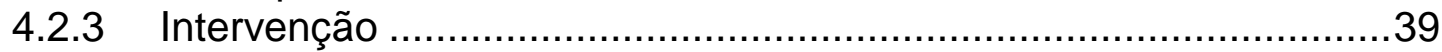

4.2.4 Comparação...................................................................... 40

4.2.5 Desfechos ..................................................................... 40

4.3 Riscos de Vieses nos Estudos Individuais ....................................42

4.4 Síntese dos Resultados (metanálises), Riscos de Vieses entre os Estudos e Análises Adicionais. 


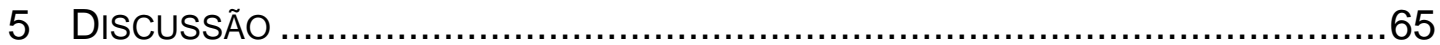

5.1 Síntese das Evidências ....................................................... 70

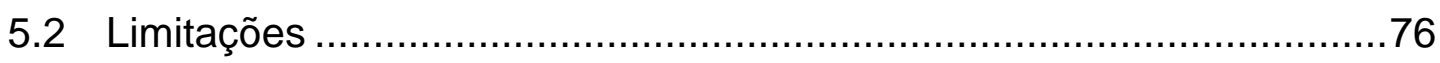

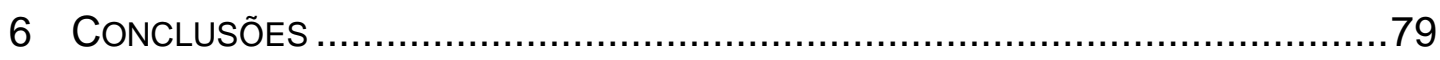

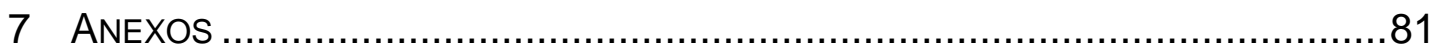

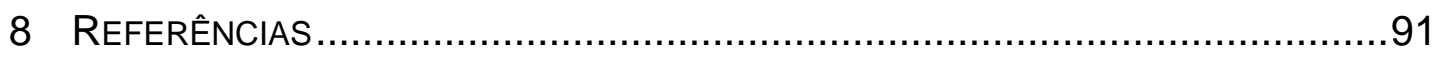

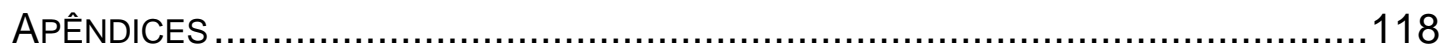




\section{LISTA DE ABREVIATURAS E SIGLAS ${ }^{1}$}

\begin{tabular}{|c|c|}
\hline AJCC & - American Joint Committee on Cancer \\
\hline ARA & - Aumento do risco absoluto \\
\hline BVS & - Biblioteca Virtual em Saúde \\
\hline CAF & - Cauterização de alta frequência \\
\hline $\mathrm{CaG}$ & - Câncer gástrico \\
\hline CAPES & $\begin{array}{l}\text { - Coordenação de Aperfeiçoamento de Pessoal de } \\
\text { Nível Superior }\end{array}$ \\
\hline CAT & - Software Critically Appraised Topic \\
\hline CEBM & $\begin{array}{l}\text { - Centro de Medicina Baseada em Evidências de } \\
\text { Oxford (Oxford Centre for Evidence-based Medicine) }\end{array}$ \\
\hline CGP & - Câncer gástrico precoce \\
\hline CINAHL & - Cumulative Index to Nursing and Allied Health Literature \\
\hline Cochrane & - Cochrane Central Register of Controlled Trials \\
\hline DR & - Diferença de risco \\
\hline EBSCO & - Elton Bryson Stephens COmpany \\
\hline EMBASE & - Excerpta Medica dataBASE \\
\hline EMR & - Mucosectomia (Endoscopic mucosal resection) \\
\hline EMR-L & $\begin{array}{l}\text { - Mucosectomia com dispositivo de ligadura } \\
\text { (Endoscopic mucosal resection with ligation device) }\end{array}$ \\
\hline ESD & $\begin{array}{l}\text { - Dissecção submucosa endoscópica (Endoscopic } \\
\text { submucosal dissection) }\end{array}$ \\
\hline IC & - Intervalo de confiança \\
\hline JGCA & - Japanese Gastric Cancer Association \\
\hline LILACS & $\begin{array}{l}\text { - Literatura Latino-Americana e do Caribe em Ciências } \\
\text { da Saúde }\end{array}$ \\
\hline MEDLINE & - Medical Literature Analysis and Retrieval System Online \\
\hline $\mathrm{NNH}$ & - Número necessário para causar dano (harm) \\
\hline NNT & - Número necessário para tratar \\
\hline
\end{tabular}

1 Algumas abreviaturas e siglas serão mantidas em inglês devido às suas consagrações pelo uso na comunidade médica e científica. 


\begin{tabular}{|c|c|}
\hline NOS & - Newcastle-Ottawa Scale \\
\hline OMS & - Organização Mundial da Saúde \\
\hline PAAF & - Punção aspirativa com agulha fina \\
\hline PET-CT com FDG & $\begin{array}{l}\text { - Tomografia com emissão de pósitrons com } \\
\text { fluordeoxiglicose }\end{array}$ \\
\hline PRISMA & $\begin{array}{l}\text { - Preferred Reporting Items for Systematic reviews and } \\
\text { Meta-analyses }\end{array}$ \\
\hline PROSPERO & $\begin{array}{l}\text { - International Prospective Register of Systematic } \\
\text { Reviews }\end{array}$ \\
\hline RA & - Risco absoluto \\
\hline RRA & - Redução do risco absoluto \\
\hline Scopus & - Base de dados da Elsevier \\
\hline SIGN & - Scottish Intercollegiate Guidelines Network \\
\hline USE & - Ultrassonografia endoscópica \\
\hline
\end{tabular}




\section{LISTA DE FIGURAS}

Figura 1- Esquema representativo da classificação de Paris para as lesões neoplásicas superficiais do trato gastrointestinal ...............9

Figura 2 - Esquema ilustrativo evidenciando os achados ecoendoscópicos de um CGP..............................................12

Figura 3 - Técnica de mucosectomia por injeção salina submucosa .........15

Figura 4 - Técnica de mucosectomia por tração (aparelho de duplo canal)

Figura 5 - Técnica de mucosectomia por sucção com auxílio de

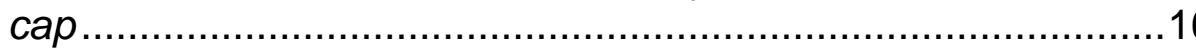

Figura 6 - Técnica de dissecção submucosa endoscópica ....................17

Figura 7 - Fluxograma de busca e seleção dos estudos .........................38 


\section{LISTA DE GRÁFICOS}

Gráfico 1 - Sobrevida de três anos após os tratamentos endoscópico e cirúrgico do câncer gástrico precoce Gráfico em floresta

Gráfico 2 - Distribuição dos seis estudos que relatam a sobrevida de 3 anos após os tratamentos endoscópico e cirúrgico do câncer gástrico precoce - Gráfico em funil.

Gráfico 3 - Sobrevida de cinco anos após os tratamentos endoscópico e cirúrgico do câncer gástrico precoce Gráfico em floresta

Gráfico 4- Distribuição dos nove estudos que relatam a sobrevida de cinco anos após os tratamentos endoscópico e cirúrgico do câncer gástrico precoce - Gráfico em funil.

Gráfico 5 - Sobrevida de cinco anos após os tratamentos endoscópico e cirúrgico do câncer gástrico precoce (excluindo-se o outlier) - Gráfico em floresta

Gráfico 6 - Distribuição dos oito estudos que relatam a sobrevida de cinco anos após os tratamentos endoscópico e cirúrgico do câncer gástrico precoce (excluindo-se o outlier) - Gráfico em funil.

Gráfico 7 - Sobrevida de cinco anos após os tratamentos endoscópico (ESD) e cirúrgico do câncer gástrico precoce - Gráfico em floresta.

Gráfico 8 - Distribuição dos quatro estudos que relatam a sobrevida de cinco anos após os tratamentos endoscópico (ESD) e cirúrgico do câncer gástrico precoce - Gráfico em funil

Gráfico 9 - Sobrevida de 10 anos após os tratamentos endoscópico e cirúrgico do câncer gástrico precoce Gráfico em floresta

Gráfico 10 - Distribuição dos dois estudos que relatam a sobrevida de 10 anos após os tratamentos endoscópico e cirúrgico do câncer gástrico precoce - Gráfico em funil. 
Gráfico 11 - Sobrevida de 10 anos após os tratamentos endoscópico e cirúrgico do câncer gástrico precoce (excluindo-se o outlier) - Gráfico em floresta

Gráfico 12 - Complicações após os tratamentos endoscópico e cirúrgico do câncer gástrico precoce - Gráfico em floresta

Gráfico 13 - Distribuição dos oito estudos que relatam as complicações após os tratamentos endoscópico e cirúrgico do câncer gástrico precoce - Gráfico em funil .... .55

Gráfico 14 - Complicações após os tratamentos endoscópico e cirúrgico do câncer gástrico precoce (excluindo-se os outliers) - Gráfico em floresta.

Gráfico 15 - Distribuição dos seis estudos que relatam as complicações após os tratamentos endoscópico e cirúrgico do câncer gástrico precoce (excluindo-se os outliers) - Gráfico em funil.

Gráfico 16 - Complicações após os tratamentos endoscópico (ESD) e cirúrgico do câncer gástrico precoce - Gráfico em floresta

Gráfico 17 - Distribuição dos cinco estudos que relatam as complicações após os tratamentos endoscópico (ESD) e cirúrgico do câncer gástrico precoce - Gráfico em funil

Gráfico 18 - Complicações após os tratamentos endoscópico (ESD) e cirúrgico do câncer gástrico precoce (excluindo-se os outliers) - Gráfico em floresta.

Gráfico 19 - Distribuição dos três estudos que relatam as complicações após os tratamentos endoscópico (ESD) e cirúrgico do câncer gástrico precoce (excluindo-se os outliers) - Gráfico em funil.

Gráfico 20 - Ressecções completas após os tratamentos endoscópico e cirúrgico do câncer gástrico precoce Gráfico em floresta .59

Gráfico 21 - Distribuição dos cinco estudos que relatam as ressecções completas após os tratamentos endoscópico e cirúrgico do câncer gástrico precoce Gráfico em funil.

Gráfico 22 - Ressecções completas após os tratamentos endoscópico e cirúrgico do câncer gástrico precoce (excluindo-se o outlier) - Gráfico em floresta 
Gráfico 23 - Distribuição dos quatro estudos que relatam as ressecções completas após os tratamentos endoscópico e cirúrgico do câncer gástrico precoce (excluindo-se o outlier) - Gráfico em funil

Gráfico 24 - Recorrências após os tratamentos endoscópico e cirúrgico do câncer gástrico precoce - Gráfico em floresta

Gráfico 25 - Distribuição dos sete estudos que relatam as recorrências após os tratamentos endoscópico e cirúrgico do câncer gástrico precoce - Gráfico em funil

Gráfico 26 - Recorrências após os tratamentos endoscópico e cirúrgico do câncer gástrico precoce (excluindo-se os outliers) - Gráfico em floresta.

Gráfico 27 - Distribuição dos cinco estudos que relatam as recorrências após os tratamentos endoscópico e cirúrgico do câncer gástrico precoce (excluindo-se os outliers) - Gráfico em funil.

Gráfico 28 - Mortalidade após os tratamentos endoscópico e cirúrgico do câncer gástrico precoce - Gráfico em floresta

Gráfico 29 - Distribuição dos quatro estudos que relatam a mortalidade após os tratamentos endoscópico e cirúrgico do câncer gástrico precoce - Gráfico em funil 


\section{RESUMO}

Kondo A. Abordagem endoscópica comparada à cirúrgica no tratamento do câncer gástrico precoce: revisão sistemática e metanálises [tese]. São Paulo: Faculdade de Medicina, Universidade de São Paulo, 2016.

Os desfechos clínicos e oncológicos dos pacientes submetidos à ressecção endoscópica do câncer gástrico precoce (CGP), considerando os critérios de indicação, comparados à cirurgia, não foram relatados em revisões sistemáticas. A pesquisa foi desenvolvida para estabelecer os desfechos de curto e longo prazos da ressecção endoscópica comparada à cirurgia no tratamento do CGP, elevando as informações para o nível de evidência 2a, melhor respaldando a prática clínica. A revisão sistemática com metanálises foi procedida utilizando-se as bases Medline, Embase, Cochrane, LILACS, Scopus e CINAHL. Onze coortes retrospectivas foram selecionadas para análise qualitativa e quantitativa. Todos os estudos incluem pacientes com CGP e comparam os desfechos nos dois braços. Os dados envolveram 2654 pacientes que preenchiam os critérios absolutos ou expandidos para ressecção endoscópica. Diferentes modalidades de tratamento endoscópico foram avaliadas, principalmente os procedimentos de ressecção, como endoscopic mucosal resection (EMR) e endoscopic submucosal dissection (ESD). As informações basearam-se nas características dos participantes, critérios de inclusão e exclusão, tipos de intervenções e desfechos (diferentes taxas de sobrevida, eventos adversos, ressecção completa, recorrência e mortalidade). As análises dos riscos absolutos dos desfechos foram feitas com o software RevMan, computando-se as diferenças de risco (DR) das variáveis dicotômicas. Dados de DR e intervalo de confiança de 95\% (IC) foram calculados utilizando-se o teste de Mantel-Haenszel e a inconsistência foi qualificada e reportada em $X^{2}$ e método Higgins $\left(I^{2}\right)$. A análise de sensibilidade foi feita quando a heterogeneidade era maior que 
$50 \%$. Todas as análises basearam-se inicialmente no modelo de efeito fixo. Dados de sobrevida de 3 anos estavam disponíveis em seis estudos ( $n=$ 1197). Não houve DR após os dois tratamentos ( $D R=0,01$, IC $95 \%=-0,02$ a 0,05$)$. A sobrevida de 5 anos $(n=2310)$ não demonstrou diferença significativa entre os grupos analisados ( $D R=0,01$, IC $95 \%=-0,01$ a 0,03). A avaliação de 551 pacientes não evidenciou desigualdade na sobrevida de 10 anos entre as diferentes abordagens ( $D R=-0,02$, IC 95\% $=-0,15$ a 0,10). Dados de complicação estavam presentes em oito estudos $(n=2439)$, e diferença significativa foi detectada ( $D R=-0,08, I C 95 \%=-0,10$ a $-0,05$ ), demonstrando melhores resultados com a endoscopia. As taxas de ressecção completa foram analisadas em 536 pacientes. Evidenciou-se diferença significativa entre o tratamento endoscópico e cirúrgico (DR = 0,13, IC $95 \%=-0,17$ a $-0,09$ ), validando melhores resultados no último grupo. A recorrência foi avaliada em cinco pesquisas $(n=1331)$ e não houve diferença entre as duas formas de terapêutica (DR $=0,01$, IC 95\% $=-0,00$ a $0,02)$. As taxas de mortalidade foram obtidas de quatro estudos $(n=1107)$, e não se evidenciou diferença entre os grupos envolvidos ( $D R=-0,01$, IC 95\% $=-0,02$ a 0,00). Conclui-se que as taxas de sobrevida de 3,5 e 10 anos, recorrência e mortalidade são semelhantes em ambos os grupos. Considerando-se as taxas de complicação, a abordagem endoscópica confere resultados mais apropriados e, analisando-se as taxas de ressecção completa, ela é inferior à cirurgia.

Descritores: Estômago. Neoplasias gástricas. Endoscopia. Endoscopia do sistema digestório. Endoscopia gastrointestinal. Gastroscopia. Gastrectomia. Procedimentos cirúrgicos operatórios. Procedimentos cirúrgicos do sistema digestório. Revisão sistemática. Metanálise. 


\begin{abstract}
Kondo A. Endoscopic approach versus surgery in the treatment of early gastric cancer: a systematic review and meta-analyses [thesis]. Sao Paulo: "Faculdade de Medicina, Universidade de São Paulo", 2016.

Clinical and oncological outcomes of endoscopic resection of early gastric cancer (EGC), considering the indication criteria, compared to surgery, have not been reported in systematic reviews. To address the short- and long-term outcomes of endoscopic resection compared to surgery in the treatment of EGC, a systematic review was performed, establishing the available data to an unpublished $2 \mathrm{a}$ strength of evidence, better handling clinical practice. A systematic review and meta-analysis using Medline, Embase, Cochrane, LILACS, Scopus and CINAHL databases were done. Eleven retrospective cohort studies were selected to quantitative and qualitative synthesis. All studies included patients diagnosed with EGC that compared outcomes considering endoscopic treatment and surgery. The included records involved 2654 patients with EGC that filled the standard or expanded indications for endoscopic resection. Different endoscopic treatment modalities were analyzed, mainly mucosal resection procedures such as endoscopic mucosal resection (EMR) and endoscopic submucosal dissection (ESD), compared to surgery. Information of the selected studies was extracted on characteristics of trial participants, inclusion and exclusion criteria, types of interventions and outcomes (different survival rates, adverse events, complete resection, recurrence and mortality rates). The analysis of the absolute risks of the outcomes was performed using the software RevMan, by computing risk differences (RD) of dichotomous variables. Data on $\mathrm{RD}$ and $95 \%$ confidence interval $(\mathrm{Cl})$ for each outcome were calculated using the Mantel-Haenszel test and inconsistency was qualified and reported in $X^{2}$ and the Higgins method $\left(\mathrm{I}^{2}\right)$. Sensitivity analysis was performed when
\end{abstract}


heterogeneity was higher than $50 \%$. All pooled analyses were initially based on fixed-effects model. Three-year survival data were available for six studies $(n=1197)$. There were no RD in 3-year survival data after endoscopic and surgical treatment of EGC (RD $=0.01,95 \% \mathrm{Cl}=-0.02$ to 0.05$)$. Five-year survival data $(n=2310)$ showed no evidence of a difference between the two groups $(\mathrm{RD}=0.01,95 \% \mathrm{Cl}=-0.01$ to 0.03$)$. The data analysis, in 551 patients, showed no difference in 10-year survival rates between the approaches ( $\mathrm{RD}=-0.02$ and $95 \% \mathrm{Cl}=-0.15$ to 0.10 ). Complication data were identified in eight studies $(n=2439)$. A significant difference was detected $(\mathrm{RD}=-0.08,95 \% \mathrm{Cl}=-0.10$ to -0.05$)$, demonstrating better results with endoscopic approach. Complete resection data was analyzed in 536 patients. It showed significant difference in complete resection rates between endoscopic and surgical treatment of EGC (RD $=-0.13,95 \% \mathrm{Cl}=-0.17$ to 0.09), exhibiting improved results in the surgical group. Recurrence data were analyzed in five studies $(n=1331)$ and there was no difference between the approaches $(\mathrm{RD}=0.01,95 \% \mathrm{Cl}=-0.00$ to 0.02$)$. Mortality data were obtained in four studies $(n=1107)$, and there was no difference between treatment modalities ( $R D=-0.01,95 \% \mathrm{Cl}=-0.02$ to 0.00 ). This systematic review concludes that 3-, 5- and 10-year survival, recurrence and mortality rates are similar for both groups. Considering procedure-related complication rates, endoscopic approach achieves significantly better results and, analyzing complete resection data, it is considered worse than surgery.

Descriptors: Stomach. Stomach neoplasms. Endoscopy. Endoscopy, digestive system. Endoscopy, gastrointestinal. Gastroscopy. Gastrectomy. Surgical procedures, operative. Digestive system surgical procedures. Review. Meta-Analysis. 
1 INTRODUÇÃo 
O câncer gástrico precoce (CGP) foi definido pela Sociedade Japonesa de Endoscopia Gastrointestinal, em 1962, como uma neoplasia que poderia ser tratada com sucesso por meio de procedimento cirúrgico. $O$ CGP, atualmente, é estabelecido, mais especificamente, como o adenocarcinoma que está restrito à camada mucosa ou submucosa da parede gástrica, independentemente de haver ou não acometimento linfonodal metastático locorregional (Murakami, 1979; Gotoda, 2006) ou à distância. De acordo com a última classificação TNM do câncer de estômago da American Joint Committee on Cancer (AJCC), aquelas lesões intituladas T1, seja qual for o status $\mathrm{N}$ e seu subtipo histopatológico, são denominadas CGP (Apêndice A). Neste estudo, considerou-se apenas o adenocarcinoma gástrico. Não foram considerados outros tipos de neoplasia como, por exemplo, linfoma ou tumores estromais.

Ressalta-se que esta definição, adotada pelo Centro Japonês Nacional do Câncer (Japanese National Cancer Centre), pela Associação Japonesa de Câncer Gástrico (Japanese Gastric Cancer Association) e outras entidades internacionais, reconhece que existe uma pequena porcentagem de pacientes com CGP que apresentam doença metastática linfonodal (Gotoda, 2006). Estes tumores têm prognóstico significativamente melhor (taxa de sobrevida de cinco anos de aproximadamente 90\%) que cânceres gástricos em estádios mais avançados. 
Globalmente, o adenocarcinoma gástrico, com cerca de um milhão de novos casos anualmente, é a terceira maior causa de mortalidade por neoplasia maligna e a primeira por câncer associado à infecção (Parkin, 2006; Ferlay et al., 2010; IACR, 2012), no caso, pela bactéria Helicobacter pylori.

O CGP compreende $15 \%$ a 57\% dos cânceres gástricos incidentais, ou seja, tumores detectados sem que haja suspeição clínica ou sintomas a ele relacionados, dependendo da região geográfica analisada (Maehara et al., 1992; Shimizu et al., 1995; Noguchi et al., 2000).

O câncer gástrico (CaG) demonstra acentuada variação geográfica quanto à sua incidência e prevalência, considerando-se regiões de um mesmo país ou entre países. Áreas de alta incidência incluem América Latina, Ásia Oriental, algumas regiões da Europa e Oriente Médio (Ferlay et al., 2010). No Japão, estima-se uma incidência anual de 78 doentes por 100.000 habitantes. Nos Estados Unidos da América, as taxas de incidência geral são modestas, porém aumentadas em grupos étnicos e raciais não-caucasianos, incluindo os hispânicos e afroamericanos (Wanebo et al., 1993; Siegel et al., 2012 e 2015). Dados estatísticos recentes revelam declínio da incidência do CaG em alguns países, especificamente nos Estados Unidos, Inglaterra e outros países mais desenvolvidos (INCA, 2015), assim como no Brasil (Zilberstein et al., 2012 e 2013b), apesar da mortalidade permanecer elevada. Por motivos desconhecidos, uma incidência crescente de CaG tem sido recentemente observada entre adultos jovens nos Estados Unidos (Anderson et al., 2010). A estimativa de novos casos de CaG no Brasil é de aproximadamente 20 mil, sendo 12 mil em homens e sete mil em mulheres (INCA, 2015), sendo São Paulo, Goiás e Pará os estados com maior número de relatos. 
A incidência de CGP, assim como a sua proporção com os adenocarcinomas gástricos de maneira geral, variam dependendo da população estudada. No Japão e Ásia Oriental, até metade das ressecções gástricas por adenocarcinoma é representada por lesões precoces. Essa proporção aumentou significativamente de $15 \%$ para até $57 \%$ após a introdução dos programas de rastreamento (Shimizu et al., 1995; Noguchi et al., 2000). No Japão, $60 \%$ dos CaG são precoces. Na Coreia, $25 \%$ a $30 \%$ dos CaG são T1 (Lee et al., 2002; Kang et al., 2010). Em países ocidentais, o CGP perfaz 15\% a 21\% dos adenocarcinomas gástricos (Eckardt et al., 1990; Everett e Axon, 1997 e 1998; Noguchi et al., 2000).

Existem várias explicações para a maior incidência de CGP na Ásia em relação a países ocidentais. As taxas de incidência de CaG são cinco a 10 vezes maiores na Ásia Oriental, o que contribui para esse fenômeno. Além disso, programas de rastreamento e detecção precoce do CaG vêm sendo desenvolvidos no Japão há algumas décadas, e mais recentemente em outras regiões do Oriente, possibilitando seu diagnóstico. Ainda, sabe-se que existe uma diferença bem estabelecida na interpretação histológica dos tecidos gástricos entre centros ocidentais e orientais (Schlemper et al., 2000), determinando divergência de observação quanto à sua natureza, se benigna ou maligna.

O adenocarcinoma gástrico do tipo intestinal progride por uma sequência de estágios anatomopatológicos, desde a mucosa normal, até a gastrite crônica, gastrite atrófica multifocal, metaplasia intestinal e, finalmente, displasia e adenocarcinoma. Uma causa subjacente de 
inflamação da mucosa gástrica e sua cronicidade é a infecção pela bactéria Helicobacter pylori, assim como a modulação da resposta do hospedeiro à sua infecção e fatores dietéticos e ambientais (Jacob et al., 2009; Wroblewski et al., 2010; Zilberstein et al., 2012 e 2013b).

A endoscopia digestiva alta (esofagogastroduodenoscopia) com biópsias sistemáticas não-dirigidas e/ou dirigidas de lesões suspeitas é o método diagnóstico de escolha para a detecção do CaG (Yantiss e Odze, 2009; de Vries et al., 2010; Correa et al., 2010; Dinis-Ribeiro et al., 2012).

A acurácia relatada da endoscopia de luz branca (convencional) para a detecção de CGP varia de 90\% a 96\%, embora alguns estudos tenham demonstrado valores mais baixos (Ballantyne et al., 1987). À endoscopia, um CGP pode apresentar-se como uma protrusão polipoide sutil, placa superficial, descoloração mucosa, depressão ou úlcera (Kajitani, 1981). A detecção de lesões discretas e de pequenas dimensões pode ser difícil, ocorrendo a inobservância dessas alterações mesmo por endoscopistas experientes. A avaliação cuidadosa e pormenorizada da totalidade da mucosa gástrica é essencial, com biópsia de quaisquer lesões suspeitas. $\mathrm{O}$ mapeamento topográfico do estômago com biópsias não-dirigidas deve ser considerado em pacientes e populações de alto risco. Em países ocidentais, essa abordagem é aceitável para indivíduos com história familiar de CaG ou metaplasia intestinal conhecida, para imigrantes de áreas de alta incidência e para pacientes com características clínicas ou endoscópicas que requeiram vigilância (Yantiss e Odze, 2009; de Vries et al., 2010; Correa et al., 2010; Dinis-Ribeiro et al., 2012). 
Os programas de rastreamento do CaG elaborados na Ásia Oriental levaram ao desenvolvimento de novas tecnologias endoscópicas em imagem, com aplicabilidade prática em todo o trato gastrointestinal. A melhoria na detecção de lesões pode ser verificada utilizando-se a cromoendoscopia, a magnificação de imagem, a tecnologia de imagem em banda estreita (do inglês narrow band imaging [NBI]) com ou sem magnificação e a autofluorescência (Muto et al., 2005; Kato et al., 2010; Tada et al., 2011; Ezoe et al., 2011; Li et al., 2012; Subramanian e Ragunath, 2014; Yao et al., 2014). A cromoendoscopia com magnificação atualmente é o padrão em vários centros de referência na Ásia Oriental.

A incidência de metástases linfonodais, de modo geral, no CGP estádio clínico T1 varia de 0\% a 15\% (Folli et al., 1995; Seto et al., 1997; Nakamura et al., 1999; Gotoda et al., 2000; Roviello et al., 2006; Lee HH et al., 2013). Apesar da presença de linfonodos comprometidos não alterar a designação de uma lesão T1 como CGP, ela tem implicação no tratamento. Pacientes com diagnóstico ou alta probabilidade clínica ou radiológica de metástase(s) linfonodal(ais) não são candidatos apropriados para a abordagem endoscópica. A gastrectomia com linfadenectomia é o tratamento de escolha nestas situações.

As características do tumor apresentam relação direta com a probabilidade de haver metástases linfonodais e tem ajudado a refinar os protocolos e diretrizes que determinam os indivíduos apropriados (candidatos) para a abordagem endoscópica (Hiki et al., 1992).

Os fatores associados à presença de metástases linfonodais incluem tumores com grandes dimensões, presença de ulceração, subtipo histológico 
difuso (indiferenciado) ou misto (intestinal/indiferenciado), profundidade de invasão na parede gástrica e acometimento submucoso ou linfovascular (Sano et al., 1992; Roviello et al., 2006; Nasu et al., 2006; An et al., 2007; Lee IS et al., 2013; Fujikawa et al., 2015). A estimativa endoscópica do tamanho da lesão é um importante fator, uma vez que seu subdimensionamento pode afetar, de modo negativo, as taxas de ressecção completa e curativa (Hiki et al., 1992; Shim et al., 2014) (Quadro 1).

Quadro 1 - Frequência de metástase linfonodal no câncer gástrico precoce conforme as características da lesão

\begin{tabular}{|l|c|c|c|c|c|c|c|}
\hline \multicolumn{7}{|c|}{ Câncer gástrico precoce (n=631) - Frequência de metástase linfonodal (\%) } \\
$\begin{array}{l}\text { Tamanho } \\
\text { (mm) }\end{array}$ & $1-5$ & $6-10$ & $11-15$ & $16-20$ & $21-25$ & $26-30$ & $>30$ \\
Tipo & 0 & 0 & 0 & 0 & 0 & 8,3 & 14,2 \\
Elevado & 0 & 0 & 0 & 0 & 6,2 & 9 & 12,1 \\
Deprimido (ul-) & 0 & 5,8 & 3,7 & 8,3 & 2,7 & 9 & 19,3 \\
Deprimido (ul+) & & & & 20 & 30 & & 23,8 \\
Misto & & & &
\end{tabular}

(ul-): não ulcerado, (ul+): ulcerado

Adaptado de Hiki et al. (1992)

Os tumores do estômago podem ser classificados de diversas maneiras, de acordo com seus achados macroscópicos e histopatológicos. Além disso, marcadores moleculares poderão melhor definir, no futuro, a sua classificação e prover informações úteis para determinar as formas de tratamento mais adequadas, assim como predizer o prognóstico de modo mais consistente.

Centros de referência elaboraram grupo para se estabelecer consenso quanto à classificação dos CGP. Foi formulada e desenvolvida a Classificação de Vienna das neoplasias epiteliais gastrointestinais (Schlemper et al., 2000) (Apêndice B). Independentemente, a Organização 
Mundial da Saúde (OMS) propôs uma classificação de neoplasias gástricas intraepiteliais utilizando termos diferentes [negativo para displasia, indefinido para displasia, displasias de baixo e alto grau, e carcinoma (invasão até a lâmina própria ou além dela)] (Fenoglio-Preiser et al., 2000).

Histologicamente, os CaG podem ser classificados, basicamente, nos subtipos intestinal, difuso ou misto, de acordo com a Classificação de Lauren (Lauren, 1965; Lauren e Nevalainen, 1993). Mais detalhadamente, de acordo com a classificação japonesa, pode-se subdividir o CaG em: adenocarcinoma papilar, tubular (bem ou moderadamente diferenciado), pouco diferenciado, mucinoso e com células em anel de sinete (JGCA, 2011a).

Alguns sistemas de classificação também foram desenvolvidos especificamente para os cânceres precoces do trato gastrointestinal. Apesar de frequentemente utilizados na Ásia, eles são pouco usados nos Estados Unidos e Europa. Particularmente, a Classificação Japonesa Macroscópica dos Carcinomas Superficiais Gástricos é amplamente aplicada na Ásia Oriental (JGCA, 1998) (Apêndice C). Essa classificação baseia-se nas características visuais e ultrassonográficas das lesões, e foi criada numa tentativa de determinar as indicações e desfechos relacionados às ressecções endoscópicas (The general rules..., 1973).

Uma nova classificação, o sistema de Paris, foi proposto em 2002 e é semelhante à classificação japonesa (The Paris endoscopic..., 2003). As lesões superficiais são categorizadas como polipoide, não polipoide ou escavadas (Figura 1): 
1. Tipo 0-I - lesões polipoides

0-Ip - pedunculada

0-Is - séssil

2. Tipo 0-II - lesões não polipoides

0-Ila - levemente elevada

0-IIb - plana

0-IIc - levemente deprimida

3. Tipo 0-III - lesões escavadas

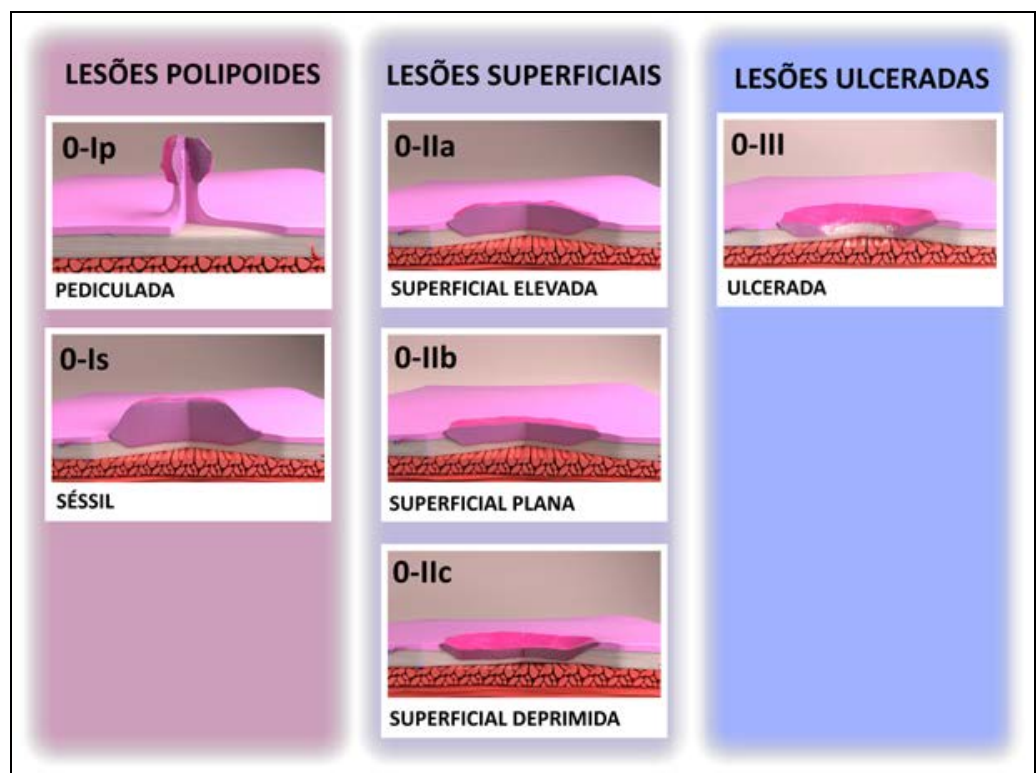

Figura 1- Esquema representativo da classificação de Paris para as lesões neoplásicas superficiais do trato gastrointestinal [adaptado de The Paris endoscopic... (2003), imagem cedida por: Gustavo Luis Rodela Silva]

O consenso de Paris confirmou a importância da Classificação Japonesa para o Câncer Gástrico, uma vez que ela se baseia na correlação com o risco de metástases linfonodais (The Paris endoscopic..., 2003).

Os achados da endoscopia podem predizer o estádio do tumor. Características encontradas em lesões intramucosas incluem leve protrusão 
da superfície mucosa, discreta elevação marginal e suave afilamento de pregas convergentes. Achados sugestivos de lesão com infiltração da camada submucosa envolvem superfície irregular, acentuada elevação ou deformidade marginal, entalhe abrupto ou fusão de pregas convergentes (Choi J et al., 2011).

Entretanto, o estadiamento final é estabelecido analisando-se o produto de ressecção, seja a peça obtida por endoscopia ou cirurgia.

A ressecção endoscópica é considerada procedimento tanto de estadiamento quanto de tratamento para o CGP. A ressecção em bloco (en bloc) permite avaliar o estádio $\mathrm{T}$ da lesão e pode indicar subsequente gastrectomia com linfadenectomia se a ressecção for incompleta ou existirem achados histopatológicos desfavoráveis. A presença destes achados sugere maior incidência de metástases linfonodais e, desta forma, indica que a abordagem cirúrgica radical é preferível (Jacob, 2003; Gunaratnam et al., 2015).

A ultrassonografia endoscópica (USE) é considerada o método não invasivo mais confiável para a avaliação da profundidade de invasão do tumor gástrico, particularmente para lesões T1 (Yoshida et al., 2005). Analisando-se a parede gástrica e suas camadas, o grau de acometimento tumoral pode ser mais adequadamente definido pela USE do que pela tomografia computadorizada de abdome (Yanai et al., 1993 e 1996) (Figura 2).

O possível envolvimento metastático de linfonodos regionais também pode ser avaliado pela USE. A definição de linfonodos regionais e extrarregionais para o CaG depende da sua localização (Edge et al., 2009). 
Muito embora a USE possa detectar o aumento dos linfonodos, a caracterização e diferenciação de um processo inflamatório perigástrico benigno (reativo) de uma metástase pode ser bastante difícil. Desta forma, a punção ecoguiada com agulha fina (PAAF) de linfonodos suspeitos pode determinar melhor acurácia no estadiamento nodal (Chang et al., 1994).

A USE é ferramenta diagnóstica de grande importância para a seleção dos pacientes com CGP candidatos à ressecção endoscópica. Porém, algumas características podem determinar um estadiamento tumoral inadequado. Lesões maiores que $3 \mathrm{~cm}$ ou localizadas no estômago médio estão sob risco de serem superestadiadas, enquanto lesões pouco diferenciadas, de serem subestadiadas. Desta forma, em muitos centros de referência asiáticos, a ressecção endoscópica, ao invés da USE, é considerada a primeira opção no estadiamento do CGP (Gotoda et al., 2006; Kim et al., 2007; Lee et al., 2016). Em contrapartida, em países ocidentais, a USE geralmente é recomendada para todos os pacientes sem evidência de doença metastática (NCCN, 2015). 


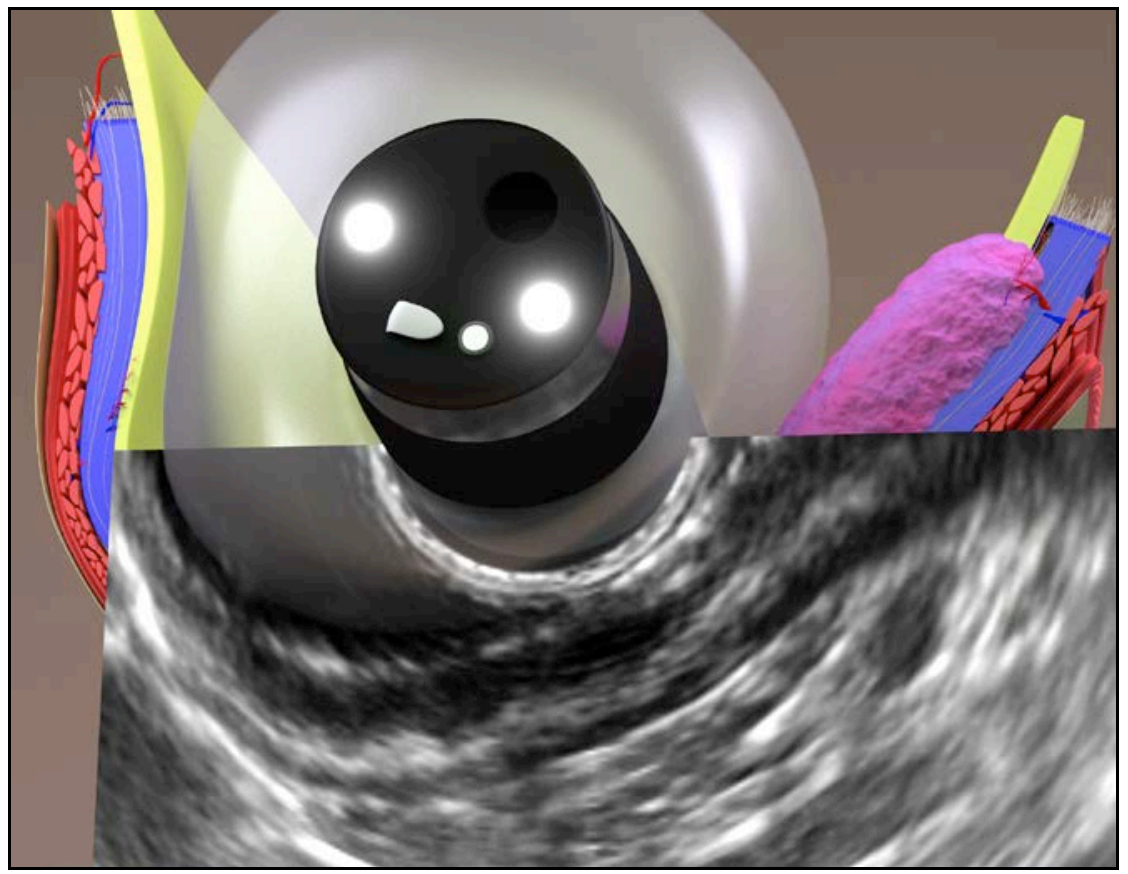

Figura 2- Esquema ilustrativo evidenciando os achados ecoendoscópicos de um CGP: tumor (rosa), camadas mucosa (amarelo), submucosa (azul), muscular própria (vermelho) e serosa (marrom) [Imagem cedida por: Gustavo Luis Rodela Silva]

Outros exames para estadiamento, mais comumente usados nos centros ocidentais, como a tomografia com emissão de pósitrons com fluordeoxiglicose (PET-CT com FDG) e tomografia computadorizada de abdome podem ser realizadas seletivamente (Tsendsuren et al., 2006; NCCN, 2015).

Os métodos de tratamento do CGP incluem, basicamente, a ressecção endoscópica e a cirurgia. As ressecções endoscópicas, seja por mucosectomia (endoscopic mucosal resection [EMR]) ou dissecção submucosa endoscópica (endoscopic submucosal dissection [ESD]), são opções para pacientes rigorosamente selecionados. Aqueles que não se enquadram nos critérios pré-estabelecidos, são encaminhados para a cirurgia. 
De acordo com as diretrizes atuais, as indicações absolutas (ou standard) para o tratamento endoscópico do CGP são definidas para o adenocarcinoma intramucoso bem ou moderadamente diferenciado que é elevado e tem até $2 \mathrm{~cm}$ de diâmetro, ou aquele que é deprimido e mede até 1 cm, sem ulceração (JGCA, 2011b). Importante ressaltar que estas lesões devem apresentar uma alta probabilidade de ressecção en bloc e não haver indícios de invasão linfovascular (Min et al., 2014). Os critérios absolutos, no entanto, são tão estritos e rigorosos que, provavelmente, cirurgias desnecessárias estão sendo realizadas (Gotoda, 2007). Assim, critérios de indicação expandidos para a ressecção endoscópica foram sugeridos (JGCA, 2011b) e vários trabalhos têm demonstrado bons e animadores resultados (Ahn et al., 2011). Gotoda (2007) propôs os critérios expandidos para a ressecção endoscópica: (a) câncer intramucoso sem ulceração, independentemente do seu tamanho; (b) câncer intramucoso $\leq 3 \mathrm{~cm}$ de diâmetro com ulceração; e (c) câncer invasivo com mínima ( $\leq 500 \mu \mathrm{m}$ da camada muscularis mucosae) invasão submucosa e tamanho $\leq 3 \mathrm{~cm}$ (Quadro 2).

\section{Quadro 2 - Esquema ilustrativo das características do CGP e seu tratamento recomendado}

\begin{tabular}{|c|c|c|c|c|c|c|}
\hline \multirow{3}{*}{ Histologia } & \multicolumn{4}{|c|}{ Câncer mucoso $(\mathrm{m})$} & \multicolumn{2}{|c|}{ Câncer submucoso (sm) } \\
\hline & \multicolumn{2}{|c|}{ Sem ulceração } & \multicolumn{2}{|c|}{ Com ulceração } & \multirow{2}{*}{$\begin{array}{c}\mathrm{sm} 1 \\
\leq 30 \mathrm{~mm}\end{array}$} & \multirow{2}{*}{$\frac{\leq \mathrm{sm} 2}{\text { independente }}$} \\
\hline & $\leq 20 \mathrm{~mm}$ & $>20 \mathrm{~mm}$ & $\leq 30 \mathrm{~mm}$ & $>30 \mathrm{~mm}$ & & \\
\hline Diferenciado & & & & & & \\
\hline \multicolumn{7}{|l|}{ Indiferenciado } \\
\hline \multicolumn{4}{|c|}{ Indicações absolutas para EMR ou ESD } & \multicolumn{3}{|c|}{ Indicações expandidas para ESD } \\
\hline \multicolumn{3}{|c|}{ Considerar cirurgia } & & \multicolumn{3}{|c|}{ Cirurgia (gastrectomia com linfadenectomia) } \\
\hline
\end{tabular}


As indicações gerais da gastrectomia com linfadenectomia incluem, basicamente, lesões com baixa possibilidade de ressecção en bloc, subtipo histológico difuso, tumores ulcerados ou com invasão submucosa maciça, evidência de acometimento linfovascular ou linfonodal.

\subsection{Mucosectomia e Dissecção Endoscópica Submucosa}

A mucosectomia foi introduzida em 1978. Quando disponível é, frequentemente, o procedimento de escolha para os pacientes que preenchem os critérios absolutos para tratamento endoscópico do CGP. Tecnicamente, trata-se de um procedimento para a remoção de lesões superficiais, pois permite a ressecção num plano entre a submucosa profunda e a camada muscular própria.

Vários acessórios e modalidades podem ser empregados. Basicamente, o método consiste em atingir o plano adequado por meio de sucção, tração da mucosa ou injeção salina submucosa, seguindo-se da ressecção com alça eletrocirúrgica endoscópica (Chaves et al., 2010). Sakai et al. (1996) descreveram a técnica de mucosectomia utilizando um dispositivo de ligadura elástica.

Para melhor entendimento, as Figuras 3, 4 e 5 exibem as formas mais comuns de EMR. 


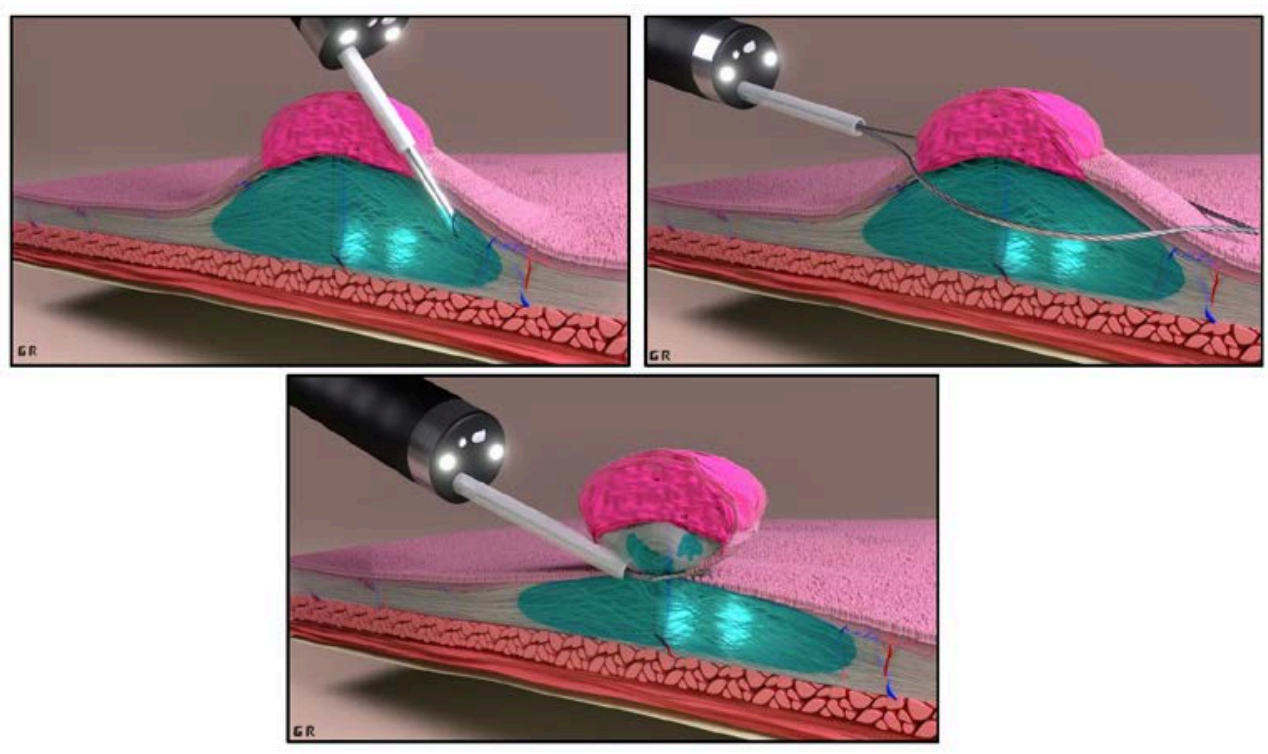

Figura 3 - Técnica de mucosectomia por injeção salina submucosa [Imagem cedida por: Gustavo Luis Rodela Silva]

As complicações imediatas deste procedimento incluem, basicamente, o sangramento e a perfuração, mesmo quando realizado por médicos treinados. É seguro e pode ser feito ambulatorialmente.

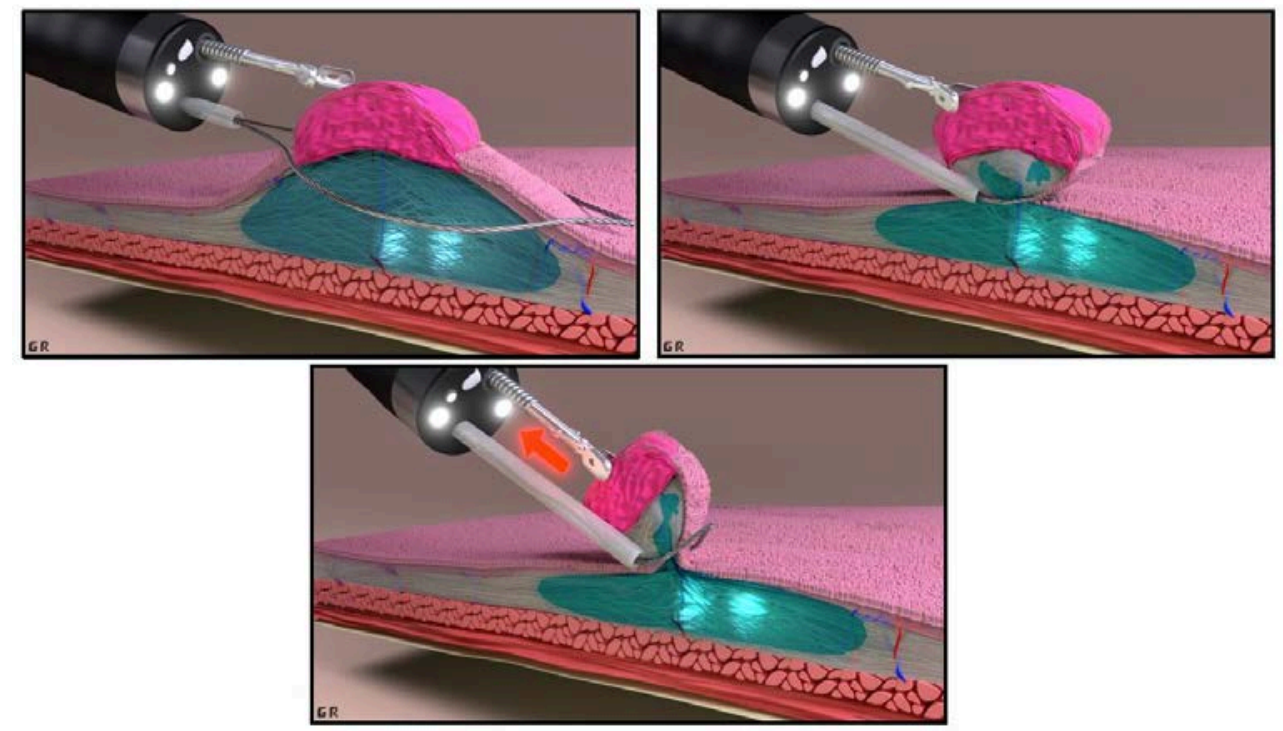

Figura 4 - Técnica de mucosectomia por tração (aparelho de duplo canal) [Imagem cedida por: Gustavo Luis Rodela Silva] 


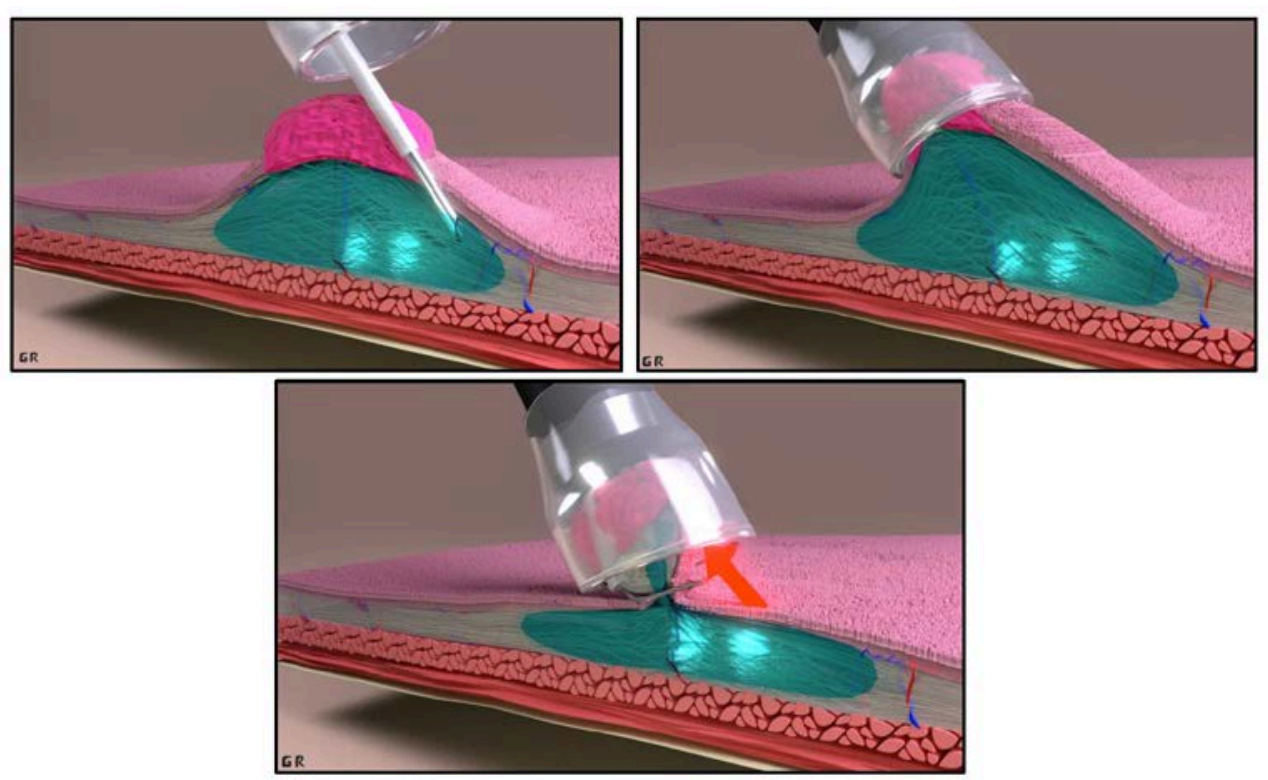

Figura 5 - Técnica de mucosectomia por sucção com auxílio de cap [Imagem cedida por: Gustavo Luis Rodela Silva]

A dissecção submucosa endoscópica, introduzida no final da década de 1990, exige centros habilitados para a sua realização, sendo mais frequentemente procedida em países asiáticos (Chiu, 2014). Tumores maiores estão sob risco de ressecções incompletas (piecemeal) com EMR, o que está associado ao aumento das taxas de recorrência. A ESD permite que a ressecção seja feita en bloc para lesões maiores, cujo tratamento por EMR pudesse ficar comprometido (Ono, 2005; Oka et al., 2006; Probst et al., 2010; Cho et al., 2011; Chaves et al., 2013). Além disso, a ESD possibilita que se consiga margens de ressecção mais profundas, importantes naqueles tumores com envolvimento submucoso.

Tecnicamente, uma incisão é realizada na mucosa circunjacente à lesão utilizando-se agulhas eletrocirúrgicas endoscópicas após a infiltração do plano submucoso com solução salina. Segue-se com a dissecção, removendo-se a mucosa e a submucosa da respectiva camada muscular, 
objetivando a ressecção en bloc (Figura 6). É um procedimento mais desafiador que a EMR, devendo ser, portanto, realizado em centros de referência. As complicações imediatas são as mesmas da EMR.
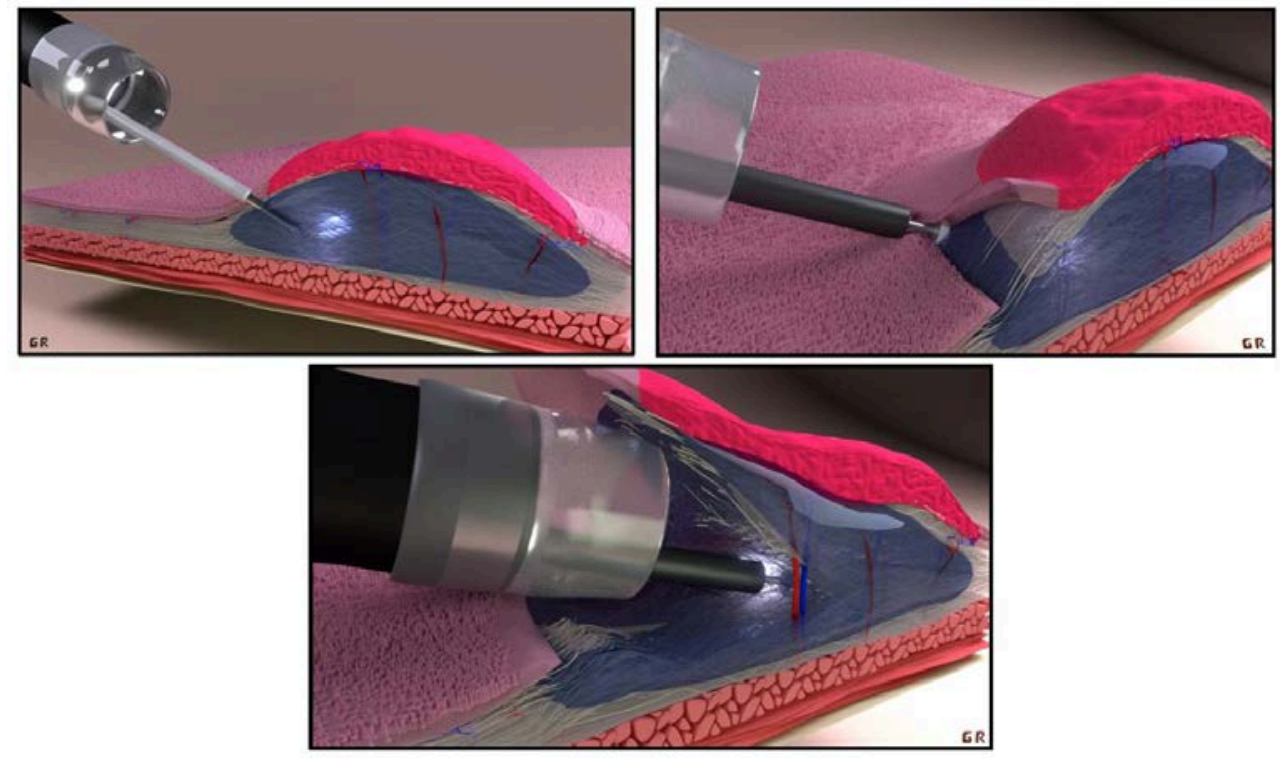

Figura 6 - Técnica de dissecção submucosa endoscópica [Imagem cedida por: Gustavo Luis Rodela Silva]

\subsection{Outras Técnicas de Tratamento Endoscópico}

Outras modalidades de tratamento endoscópico foram investigadas, mas não apresentam um papel bem estabelecido no tratamento do CGP. Essas técnicas incluem a terapia fotodinâmica (Mimura et al., 1996; Ell et al., 1998; Nakamura et al., 2001), o tratamento com Nd:YAG laser (Yasuda et al., 1993; Oguro, 1994; Sibille et al., 1995) e a coagulação com plasma de argônio (Kitamura et al., 2006; Tomita et al., 2011).

Uma desvantagem observada é que estas técnicas são consideradas métodos ablativos e não disponibilizam peças para a análise anatomopatológica, ao passo que a EMR e ESD são métodos de ressecção, que propiciam análise adequada do espécime removido (Yamamoto e Kita, 2005). 


\subsection{Cirurgia}

Os métodos de ressecção endoscópica são úteis no tratamento de pacientes que preenchem os critérios de indicação para o procedimento e que estão em centros com recursos e infraestrutura apropriados. Entretanto, globalmente, a gastrectomia permanece como o método mais utilizado no tratamento dos pacientes com CGP, atingindo sobrevidas de até $98 \%$ (Mochiki et al., 2005; Lee et al., 2009). Em casos avançados e sem perspectiva de cura, a cirurgia tem o intuito de tratar complicações (sangramento, perfuração ou obstrução) ou melhorar a capacidade de ingestão de alimentos e a qualidade de vida.

A gastrectomia é recomendada para pacientes que não preenchem os critérios para a ressecção endoscópica ou quando não existem infraestrutura e recursos humanos adequados para sua realização com segurança. Os indivíduos que não se enquadram nas indicações de tratamento endoscópico apresentam aumento do risco de metástases linfonodais e devem ser encaminhados para a cirurgia, seja aberta ou por via menos invasiva (laparoscópica ou robótica). Segundo o algoritmo proposto pela Associação Japonesa de Câncer Gástrico (JGCA), considerando-se CGP M0, os tumores cT1a, sem evidência de comprometimento linfonodal, diferenciados, menores que $2 \mathrm{~cm}$ e sem ulceração, deveriam ser tratados por endoscopia. As outras lesões cT1a(m) e as $\mathrm{cT} 1 \mathrm{~b}(\mathrm{sm})$ diferenciadas e menores que 1,5 cm, necessitariam tratamento cirúrgico com gastrectomia e linfadenectomia (D1 ou D1+). Havendo evidência de metástase(s) linfonodal(ais), recomendar-se-ia a linfadenectomia D2 (JGCA, 2011b). 
Os acessos minimamente invasivos vêm ganhando importância no tratamento das lesões malignas do aparelho digestivo (Bresciani et al., 2004 e 2007). Inicialmente, o acesso laparoscópico foi utilizado no tratamento do CGP em protocolos de estudo de instituições orientais, principalmente do Japão e Coreia do Sul, e mostrou, na ocasião, resultados promissores (Kitano et al., 2007; Yang et al., 2013). A experiência com os métodos menos invasivos (laparoscópico e robótico) vem crescendo nos últimos anos em vista dos bons resultados observados (Zilberstein et al., 2013a; Barchi et al., 2016). Wang et al. (2014) publicaram uma metanálise demonstrando a efetividade da laparoscopia e propondo estudos multicêntricos randomizados.

Apesar do acesso minimamente invasivo ser interessante, principalmente na manutenção da integridade da parede abdominal, a localização do CaG pode ser difícil, resultando em remoção excessiva de tecido normal (Lee et al., 2015).

Diversos autores propõem o acesso videolaparoscópico como o padrão-ouro no tratamento do CGP, com viabilidade e qualidade oncológicas comparáveis à cirurgia aberta (Alves et al., 2011; El-Sedfy et al., 2014; Lu et al., 2016). Dificuldades inerentes à videolaparoscopia têm dificultado a propagação da gastrectomia laparoscópica para o tratamento do câncer e, apesar dos avanços tecnológicos, ela ainda representa um procedimento desafiador. A cirurgia robótica aparenta possibilitar dissecção linfonodal de qualidade, com resultados equiparáveis aos acessos laparoscópico e aberto. Entretanto, estudos de qualidade ainda precisam ser realizados para confirmar essa impressão (Caruso et al., 2016). 


\subsection{Rationale}

Apesar dos avanços no diagnóstico e tratamento, o prognóstico dos pacientes com CaG permanece obscuro e ruim, habitualmente atribuído à fase avançada da doença à época da sua detecção.

Enquanto a gastrectomia radical pode atingir adequado tratamento oncológico por meio de margens de ressecção amplas e dissecção linfonodal (linfadenectomia), por outro lado ela impõe índices não desprezíveis de morbidades perioperatórias e mutilação que compromete as funções gastrointestinais a longo prazo, assim como a qualidade de vida dos pacientes (Gama-Rodrigues, 1986; Folli et al., 2001; Zilberstein et al., 2004; Jacob et al., 2006; Andreollo et al., 2011).

Devido aos baixos índices de metástases linfonodais do CGP, baixa invasividade, menor custo de tratamento, melhor qualidade de vida comparados à cirurgia e rápida recuperação, foram desenvolvidos métodos de tratamento endoscópico para pacientes com CGP (Soetikno et al., 2003).

Os desfechos clínicos e oncológicos de pacientes com CGP submetidos à abordagem endoscópica, considerando-se os critérios de indicação, comparados àqueles relacionados à abordagem cirúrgica, não foram relatados em revisões sistemáticas.

Essa revisão sistemática da literatura médica com metanálises foi desenvolvida para estabelecer os desfechos de curto e longo prazos dos pacientes com CGP submetidos às duas modalidades terapêuticas. 


\section{Objetivos}




\subsection{Primários}

Comparar os desfechos clínicos e oncológicos dos pacientes submetidos às diferentes abordagens (endoscópica e cirúrgica) quanto às taxas de sobrevida de três, cinco e 10 anos.

\subsection{Secundários}

Comparar os desfechos clínicos e oncológicos dos pacientes submetidos às diferentes abordagens (endoscópica e cirúrgica) quanto às complicações (basicamente, sangramento e perfuração), ressecção completa, recorrência e mortalidade. 
3 MÉtodos 
A revisão sistemática consiste numa forma de análise secundária, consistente, confiável e não tendenciosa, que visa resumir e confrontar as evidências existentes na literatura sobre determinado assunto de modo a, por meio das metanálises, integrar os resultados dos diferentes estudos, obtendo-se uma estimativa global de efeito.

Assim, foi realizada uma revisão sistemática da literatura médica em busca de artigos que comparam o tratamento endoscópico com o cirúrgico do CGP e elaboraram-se metanálises dos dados e desfechos disponíveis e passíveis de comparação.

\subsection{Protocolo e Registro}

Esta revisão sistemática da literatura foi realizada de acordo com as recomendações do Preferred Reporting Items for Systematic reviews and Meta-analyses (PRISMA) (Liberati et al., 2009). O estudo foi registrado na base de dados International Prospective Register of Systematic Reviews (PROSPERO) ${ }^{2}$, do Centro de Revisões e Disseminação (Centre for Reviews and Dissemination) da Universidade de York (Inglaterra) sob o código CRD42014015127 (PROSPERO, 2014).

2 Disponível em: <http://www.crd.york.ac.uk/prospero/>. 


\subsection{Critérios de Elegibilidade}

a) Tipos de estudos - Ensaios clínicos e/ou estudos observacionais de qualidade metodológica adequada foram pesquisados e selecionados para inclusão no processo seletivo subsequente.

b) Tipos de participantes - Pacientes com diagnóstico de CGP.

c) Tipos de intervenções - Estudos comparando desfechos em dois braços: tratamento endoscópico e cirúrgico. Não houve restrições considerando-se as diferentes modalidades de tratamento em cada braço.

d) Tipos de desfechos avaliados - Basicamente, os desfechos avaliados foram: as taxas de sobrevida de três, cinco e 10 anos, complicações, ressecção completa, recorrência e mortalidade.

\subsection{Bases de Dados}

Os estudos (ensaios clínicos e/ou estudos observacionais) foram identificados de duas maneiras: rastreando-se as bases de dados eletrônicas e procurando-se por outros artigos (não identificados na procura nas bases estabelecidas) nas listas de referências dos trabalhos selecionados.

Inicialmente, não houve restrições quanto ao idioma, tampouco na avaliação dos artigos quanto a seu status (online, impresso e outros), formato ou tipo de publicação. A busca envolveu a base de dados Medical Literature Analysis and Retrieval System Online (Medline) (considerando-se todos os anos), Excerpta Medica dataBASE (Embase) (considerando-se todos os anos), Cochrane Central Register of Controlled Trials (Cochrane), Literatura Latino-Americana e do Caribe em Ciências da Saúde (LILACS) 
(via Biblioteca Virtual em Saúde [BVS]), Base de dados da Elsevier (Scopus)

e Cumulative Index to Nursing and Allied Health Literature (CINAHL) (via Elton Bryson Stephens COmpany [EBSCO]), Coordenação de Aperfeiçoamento de Pessoal de Nível Superior (CAPES) e literatura cinzenta (expressão que designa documentos não convencionais, como por exemplo, relatórios técnicos e de pesquisa, dissertações, teses e literatura originada de encontros científicos). A última busca manual foi realizada em 20 de janeiro de 2015. Atualizações automáticas do Medline, baseadas na estratégia de busca e identificando potenciais novos estudos, foram analisadas até abril de 2015.

\subsection{Pesquisa}

A estratégia de busca discriminada abaixo, e estratificada pelos componentes População (P), Intervenção (I) e Comparação (C), foi utilizada na base de dados Medline e foi nomeada MAINMEDLINE com intuito de abreviação:

P: “\{[Gastric cancer (MeSH)] OR [(Adenocarcinoma OR Carcinoma OR Early Detection of Cancer OR Carcinoma in Situ OR Polyps) AND (Stomach OR Gastric)] OR [(Gastric Mucosa OR Early Diagnosis) AND (Gastric Cancer)]\} AND I: [Endoscopy (MeSH) OR Endoscopy, Digestive System (MeSH) OR Endoscopy, Gastrointestinal (MeSH)] AND C: [Gastrectomy (MeSH) OR Digestive System Surgical Procedures (MeSH) OR Surgery (Subheading) OR General Surgery (MeSH) OR Minimally Invasive Surgical Procedures (MeSH)]". 
A descrição detalhada dos termos MeSH (Medical Subject Headings) é fornecida no Apêndice D.

Objetivando-se a seleção de estudos de alta qualidade metodológica, a estratégia de busca mencionada acima (MAINMEDLINE) foi filtrada duas vezes (\#1 e \#2), como se segue:

\#1 “(MAINMEDLINE AND random*)"; e

\#2 “(MAINMEDLINE AND ((clinical[Title/Abstract] AND trial[Title/Abstract]) OR clinical trials as topic[MeSH Terms] OR clinical trial[Publication Type] OR random*[Title/Abstract] OR random allocation[MeSH Terms] OR therapeutic use[MeSH Subheading]) OR comparative study OR comparative studies)". A estratégia de busca \#2 é a descrição detalhada da procura quando se aplica o filtro "therapy/broad"[filter].

A totalidade dos artigos foi obtida combinando-se as estratégias de investigação e procura no Pubmed Advanced Search Builder da seguinte maneira: "(\#1 OR \#2)." Como parte de todo o processo de revisão proposto, os métodos de busca foram duplamente verificados, ou seja, pareados, por dois autores-revisores.

A tática de busca na base de dados Embase foi resumida: "((gastric cancer AND endoscopy AND surgery) AND ('clinical trial' OR 'controlled study' OR 'major clinical study' OR 'prospective study' OR 'retrospective study'))".

Para os bancos de dados científicos da Cochrane, LILACS, Scopus e CINAHL, a estratégia de procura foi: "(gastric cancer AND endoscopy AND surgery)". 


\subsection{Seleção dos Estudos}

A avaliação da elegibilidade e a seleção dos artigos rastreados foi realizada independentemente, de modo padronizado e não-cego, por dois revisores. Discordâncias entre os revisores foram resolvidas por consenso. Como as bases de dados analisadas apenas aceitam resumos nos idiomas inglês, português ou espanhol, artigos sem, ao menos, o resumo nestes idiomas foram excluídos deste trabalho. Também, relatos de casos, artigos de revisão, comentários, editoriais e cartas não foram considerados.

Inicialmente, a maioria dos estudos foi excluída pela obtenção de informações claras, no título ou no resumo, de que eles não contemplavam a comparação das abordagens endoscópica e cirúrgica no tratamento do CGP.

De modo a ilustrar o processo de seleção dos estudos, foi utilizado um fluxograma adaptado das recomendações PRISMA (Liberati et al., 2009).

\subsection{Processo de Coleta de Dados}

O método de extração de dados de cada estudo incluso consistiu no preenchimento de folhas informativas padronizadas e apropriadas após a leitura de cada artigo. Uma lista de verificação baseada nas normas e instruções da Scottish Intercollegiate Guidelines Network (SIGN, 2014) foi adquirida na Internet $^{3}$ (Anexo A). Os dados relevantes foram, então, obtidos de cada estudo incluído, utilizando-se um formulário padronizado de aquisição de informações (Anexo B). Um autor-revisor extraiu os dados científicos e um

3 Disponível em: <http:// www.sign.ac.uk>. 
segundo autor-revisor verificou as informações adquiridas. Discordâncias foram resolvidas por discussão e consenso entre os autores-revisores.

\subsection{Itens dos Dados}

As informações foram obtidas de cada estudo analisando-se os seguintes aspectos: (a) características dos participantes do estudo (incluindo a idade e padrões dos diferentes tipos de CGP) e seu critério de inclusão e exclusão, (b) tipos de intervenção (considerando-se diferentes modalidades ou combinação de técnicas no braço do tratamento endoscópico: mucosectomia, irradiação à laser, cauterização de alta frequência (CAF), ESD; versus a abordagem cirúrgica: gastrectomia ajustada ou adaptada, de acordo com o estádio do tumor, associada à linfadenectomia, se necessária) e (c) avaliação dos desfechos relacionando-os ao tempo de seguimento (diferentes curvas de sobrevida, taxas de complicações, recorrência, ressecção completa e mortalidade).

As taxas de sobrevida geral de três, cinco e 10 anos incluíram pacientes vivos no período de tempo analisado (follow-up), independentemente da presença ou ausência da doença primária.

Morbidade e complicações relacionadas aos tratamentos abrangeram quaisquer efeitos ou implicações adversas, não esperadas, decorrentes dos procedimentos.

Ressecção completa foi considerada a total remoção do CaG, utilizandose da abordagem cirúrgica ou endoscópica, determinando provável ausência de células tumorais viáveis residuais no sítio de procedimento. 
Recorrência foi caracterizada como o reaparecimento do CaG, após o seu tratamento e depois de um período de não detecção clínica e/ou radiológica. Nesta análise, foi considerada tanto a recidiva local (cicatriz da ressecção endoscópica ou anastomose cirúrgica) quanto à distância. Neste cenário, a presença de CaG metacrônico não foi contemplada.

Os dados de mortalidade baseiam-se nas mortes ocorridas e diretamente relacionadas ao procedimento proposto.

\subsection{Riscos de Vieses nos Estudos Individuais}

Para se determinar a validade e qualidade metodológica dos estudos selecionados, dois revisores mediram, de modo independente e com adequado nível de confiança, os riscos de vieses utilizando-se a Escala de Qualidade de Newcastle-Ottawa (NOS) para estudos de coorte (Ottawa Hospital Research Institute, 2015) (Anexo C) e a lista de verificações da rede SIGN (2014).

Os componentes e características metodológicas específicas foram classificados e categorizados: representatividade da população estudada (coorte exposta), seleção da coorte não exposta (ou grupo comparação), verificação adequada e análise do tipo de exposição (abordagem utilizada), demonstração de que os desfechos a serem avaliados não estavam presentes no início do estudo, comparabilidade dos grupos quanto ao desenho e métodos aplicados, avaliação dos desfechos selecionados e tempo e adequação do seguimento (follow-up).

A avaliação crítica dos estudos incluídos deveria revelar uma pontuação maior ou igual a 6, de uma pontuação máxima possível de 9. 
Os níveis de evidência de acordo com o Centro de Medicina Baseada em Evidências de Oxford (Oxford Centre for Evidence-based Medicine) (CEBM, 2009) foram obtidos (Anexo D). Essas informações foram aplicadas subsequentemente na síntese dos dados.

\subsection{Medidas de Síntese e Análise}

A medida primária dos desfechos foi a análise dos riscos absolutos das taxas de sobrevida após o tratamento do CGP. As mensurações secundárias foram determinadas ulteriormente, considerando-se as taxas de complicações, ressecção completa, recorrência e mortalidade.

Também, informações sobre redução do risco absoluto (RRA) ou aumento (ARA) e número necessário para tratar (NNT) e causar dano (NNH) foram obtidas nos desfechos principais, com intervalos de confiança (IC) de $95 \%$. 


\subsection{Método Planejado de Análise}

A análise dos dados foi realizada utilizando-se o software Review Manager (RevMan) $5.3^{4}$.

Computaram-se as diferenças de risco (DR) das variáveis dicotômicas, ou categóricas, aplicando-se o modelo de efeito fixo, que pressupõe que o efeito de interesse é o mesmo em todos os estudos e que as diferenças observadas entre eles são devidas apenas a erros amostrais (variabilidade dentro dos estudos), e obtendo-se os respectivos gráficos (forest e funnel plots, explicados detalhadamente mais adiante). Em algumas situações, utilizou-se o modelo de efeito aleatório, ou randômico, que pressupõe que o efeito de interesse não seja o mesmo em todos os estudos (considera que os estudos que fazem parte da metanálise formam uma amostra aleatória de uma população hipotética de estudos, sendo, os efeitos, conectados através de uma distribuição de probabilidade, geralmente suposta normal (Rodrigues e Ziegelmann, 2010). A utilização do modelo de efeito randômico leva em consideração o baixo poder estatístico dos testes de inconsistência e a possibilidade da existência de heterogeneidade clínica mesmo na impossibilidade de se demonstrar heterogeneidade estatística (Sedgwick, 2015).

Informações relacionadas às diferenças de risco e IC 95\% para cada desfecho foram calculadas aplicando-se o teste de Mantel-Haenszel, útil para avaliar as consequências de uma exposição (no caso, cirurgia ou endoscopia) e fornecendo uma medida global de risco entre as abordagens avaliadas.

4 (RevMan) [Computer program]. Version 5.3. Copenhagen: The Nordic Cochrane Centre, The Cochrane Collaboration, 2014. Disponível em:<http://tech.cochrane.org/revman>. 
A heterogeneidade, ou inconsistência, considerada a variabilidade ou diferença entre estudos em relação à estimativa de efeitos, foi qualificada e reportada em Chi-quadrado $\left(X^{2}\right)$ e o método de Higgins, denominado $\mathrm{I}^{2}$.

O teste do $X^{2}$ avalia se as diferenças observadas nos resultados são compatíveis apenas com chance, isto é, compara a distribuição de diversos acontecimentos em diferentes amostras, a fim de avaliar se as proporções observadas destes eventos mostram ou não diferenças significativas ou se as amostras diferem significativamente quanto às proporções desses acontecimentos. Ele fornece uma medida útil para se determinar a heterogeneidade, porém não a gradua (Higgins e Green, 2011).

O método de Higgins, denominado $\mathrm{I}^{2}$, é calculado pela fórmula $\mathrm{I}^{2}=$ $100 \% \times(Q-d f) / Q$, onde Q é o valor $X^{2}$ e df é denominado degrees of freedom (df, graus de liberdade) (Higgins e Green, 2011).

A principal vantagem desta última medida $\left(I^{2}\right)$ é que ela não depende, inerentemente, do número de estudos analisados, além de ser uma expressão intuitiva e simples da inconsistência entre as pesquisas analisadas.

Higgins et al. (2003) sugerem uma escala em que um valor de $\mathrm{I}^{2}$ próximo a 0\% indica não heterogeneidade entre os estudos, próximo a 25\% indica baixa heterogeneidade, próximo a 50\% indica heterogeneidade moderada e próximo a 75\% indica alta heterogeneidade entre os estudos.

Os dados de RRA, ARA, NNT e NNH foram obtidos para validação, após aplicabilidade testada no software Critically Appraised Topic (CAT) (Centre for Evidence-Based Medicine, 2015). 


\subsection{Riscos de Vieses entre os Estudos}

Os riscos de vieses de publicação entre os estudos, para os desfechos a serem comparados, foram plotados (funnel plots) e identificados (detecção dos outliers, estudos atípicos, diferentes da maioria, que implicam, tipicamente, em prejuízos à interpretação dos resultados), em conjunto com a análise quantitativa do $\mathrm{I}^{2}$.

\subsection{Análises Adicionais}

Assumiu-se o valor de corte de $50 \%$ para a heterogeneidade, considerando-se um número adequado para avaliação dos dados desta metanálises.

De modo a determinar se os resultados deste estudo são considerados confiáveis e apropriados para a prática médica, e não arbitrários ou baseados em dados obscuros, procedeu-se a análise de sensibilidade quando o valor da heterogeneidade $\left(\mathrm{I}^{2}\right)$ era maior que $50 \%$.

Subsequentemente, uma nova análise foi realizada, excluindo-se os outliers, e outros achados foram compilados. Uma vez confirmado que não houve modificação do status da evidência após essa análise (favorável, desfavorável ou ausência de diferença), e havendo manutenção de elevada heterogeneidade, o resultado original com todos os artigos foi mantido.

Presumiu-se heterogeneidade verdadeira quando não se detectaram outliers, isto é, viés de publicação foi excluído. Assim, as possíveis causas, neste panorama, poderiam ser: o tamanho do efeito diferindo de acordo com o tamanho da população estudada, a intensidade da intervenção e as diferenças de risco basal. 


\subsection{Aspectos Éticos}

O projeto foi aprovado pelo Comitê de Ética e Pesquisa da Faculdade de Medicina - Universidade de São Paulo, Projeto nº 037/15 (Anexo E).

As imagens foram cedidas pelo Dr. Gustavo Luis Rodela Silva, conforme Termo de Cessão de Direitos Autorais das Ilustrações (Anexo F). 
4 Resultados 


\subsection{Seleção dos Estudos}

Seis mil, trezentos e oitenta e seis estudos foram mapeados e os artigos identificados como elegíveis foram selecionados após a leitura do título e do resumo. Seis mil, trezentos e setenta e seis artigos foram excluídos por não contemplarem a comparação das diferentes abordagens em questão. Dez estudos comparavam o tratamento endoscópico versus o cirúrgico nos pacientes com CGP. Realizando-se uma varredura nas listas de referências bibliográficas dos estudos selecionados, dois outros artigos foram encontrados [Fukase et al. (1994) e Etoh et al. (2005)]. Estes trabalhos não foram identificados inicialmente na estratégia de busca adotada por duas razões. O estudo publicado por Fukase et al., em 1994, não tinha sua revista (Digestive Endoscopy) indexada até então. O artigo escrito por Etoh et al., em 2005, seria encontrado caso o filtro metodológico do Medline "epidemiologic methods"[filter], não utilizado, fosse aplicado. Um estudo de séries de casos clínicos foi excluído (Teixeira et al., 1992).

Onze artigos foram incluídos para a realização da síntese quantitativa e qualitativa.

Um fluxograma adaptado do protocolo PRISMA ilustra o processo de seleção dos estudos (Liberati et al., 2009) (Figura 7). 


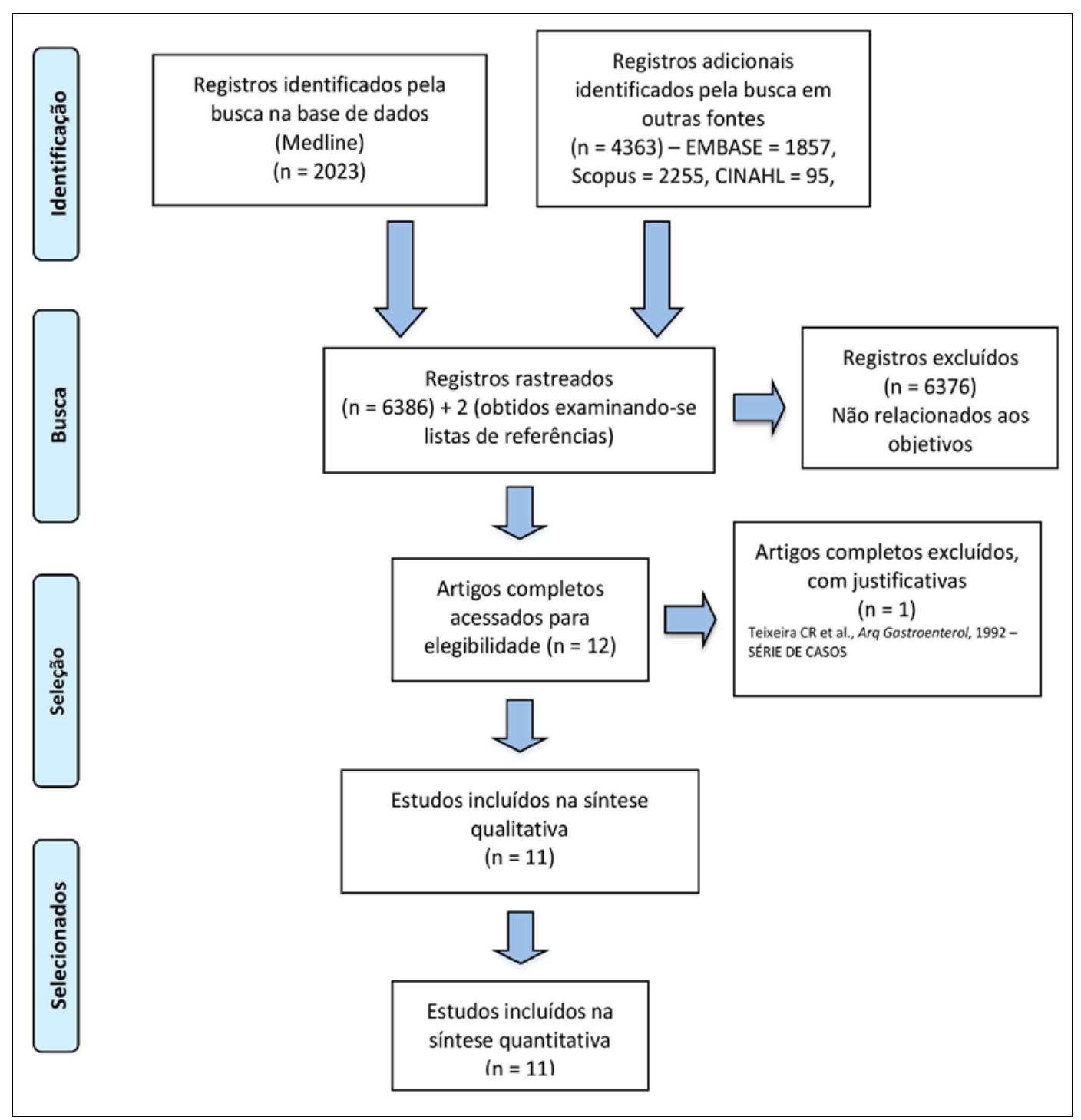

Figura 7 - Fluxograma de busca e seleção dos estudos 


\subsection{Características dos Estudos}

\subsubsection{Métodos}

Os 11 estudos selecionados para a revisão são coortes retrospectivas. Dez foram publicadas em inglês e a outra, em japonês (traduzida). Não foram identificados ensaios clínicos randomizados, controlados ou prospectivos.

\subsubsection{Participantes}

Os registros obtidos envolviam 2654 pacientes. Os critérios de inclusão para este trabalho compreendiam adultos com diagnóstico de CGP que preenchiam os preceitos de ressecção endoscópica, fossem eles absolutos ou expandidos. Uma vez que o número de estudos selecionados foi pequeno, todos os períodos de seguimento foram considerados. Os estudos publicados por Nishida et al. (1993) e Fukase et al. (1994) consideram a curva de sobrevida obtida pelo método de Kaplan Meier.

\subsubsection{Intervenção}

Diferentes modalidades de tratamento endoscópico foram analisadas, principalmente os procedimentos de ressecção endoscópica, como a mucosectomia e a dissecção submucosa endoscópica. Observou-se também a realização de cauterização de alta frequência e mucosectomia com dispositivo de sucção e ligadura. 


\subsubsection{Comparação}

Modalidades adaptadas (tailored gastrectomy) de tratamento cirúrgico para cada tipo de CGP foram comparadas à abordagem endoscópica. Os procedimentos compreendiam a gastrectomia associada ou não à linfadenectomia.

\subsubsection{Desfechos}

Os desfechos primários avaliados foram as taxas de sobrevida de curto e longo prazo, ou seja, três, cinco e 10 anos.

Os desfechos secundários e adicionais consistiam nas taxas de complicação, ressecção completa, recorrência e mortalidade.

Uma síntese das características dos estudos incluídos é mostrada na Tabela 1. 


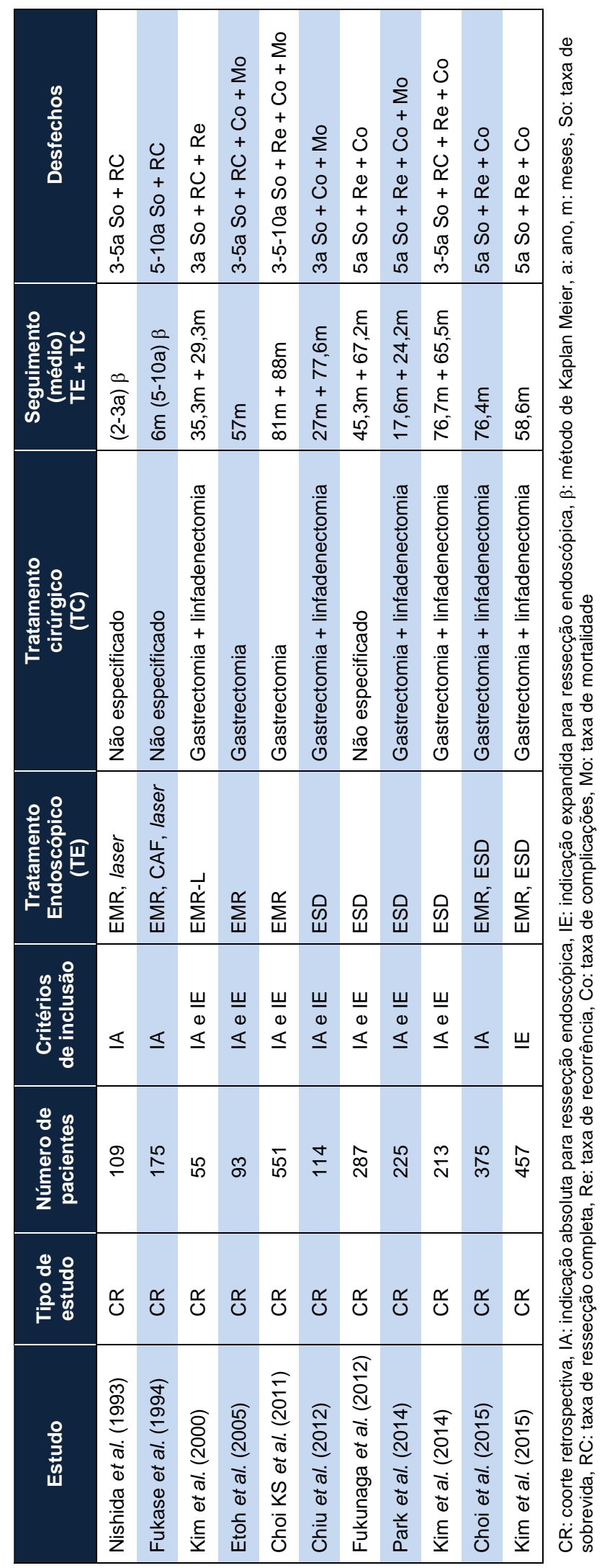




\subsection{Riscos de Vieses nos Estudos Individuais}

Utilizando-se de abordagem padronizada com critérios bem estabelecidos, como mencionado previamente, os riscos de vieses foram avaliados para definir a qualidade metodológica dos artigos selecionados. Dados de cada estudo eleito e os respectivos níveis de evidência são mostrados abaixo (Tabela 2).

Dois estudos obtiveram escore 7 de acordo com a escala NewcastleOttawa Quality Assessment Scale (Ottawa Hospital Research Institute, 2015) para estudos de coorte. Outros dois adquiriram 8 pontos e sete estudos atingiram 9 pontos. Os critérios e quesitos nos quais os respectivos estudos não alcançaram o máximo de duas estrelas (pontos), determinaram a diminuição de sua avaliação quanto à qualidade metodológica (pontuação destacada em verde na Tabela 2). 


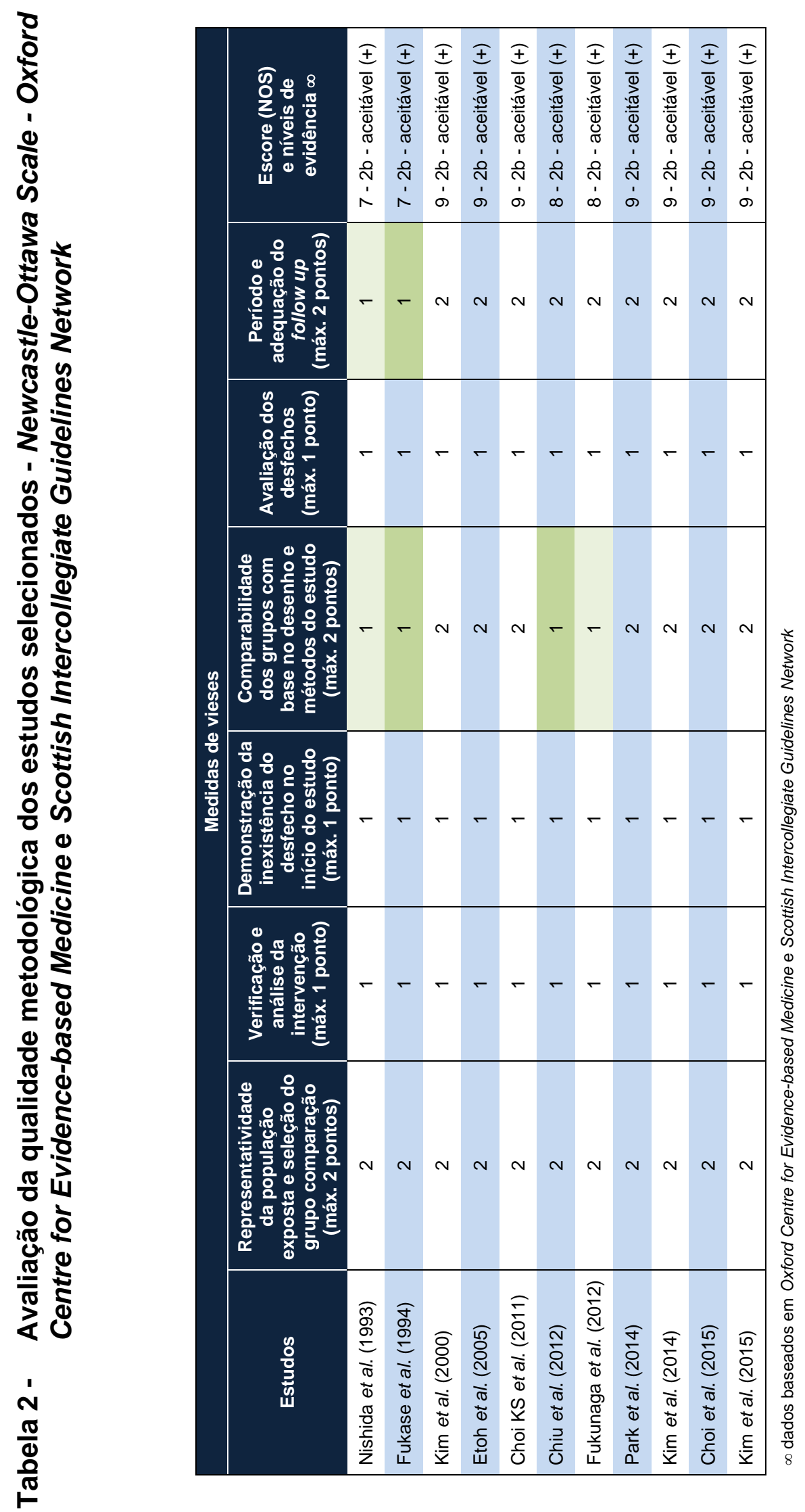


Objetivando-se aumentar a confiabilidade em relação à força da associação entre a exposição (diferentes abordagens) e os desfechos, identificando-se os aspectos de adequada qualidade metodológica, outros parâmetros também foram utilizados.

De acordo com a lista de verificação (checklist) do Scottish Intercollegiate Guidelines Network (SIGN, 2014), todos os estudos foram classificados como aceitáveis, ou seja, apresentando a possibilidade de exibir algumas falhas e um risco associado de vieses (possibilidade de mudança nas conclusões na dependência de estudos adicionais).

A classificação SIGN considera três níveis: 0 (baixa qualidade), + (aceitável) e ++ (alta qualidade). Os estudos incluídos foram classificados como tendo nível de evidência 2b, de acordo com CEBM, (2009) (Anexo D).

\subsection{Síntese dos Resultados (metanálises), Riscos de Vieses entre os Estudos e Análises Adicionais}

A síntese dos resultados é demonstrada graficamente por diagrama, denominado forest plot.

Os forest plots, ou gráficos em floresta, são a representação gráfica das metanálises. Para melhor entendimento, observa-se, à esquerda, uma tabela exibindo os estudos selecionados (autor e data), assim como os números absolutos de indivíduos em cada braço de tratamento (endoscópico e cirúrgico), considerando a quantidade de eventos (desfechos) e o total de pacientes analisados. O peso de cada estudo é mostrado em porcentagem e está diretamente relacionado ao número de indivíduos dos trabalhos. A 
tabela também lista as diferenças de risco absoluto (RA) e seus respectivos intervalos de confiança de 95\%. À direita dos diagramas, visualizam-se os forests propriamente ditos. Cada estudo é representado por uma linha horizontal, determinada pelos limites inferior e superior do IC 95\%, e por um quadrado, cujo centro (projetado verticalmente na linha graduada da parte inferior do gráfico) determina a DR (positiva ou negativa) e cujo tamanho representa o seu peso frente aos demais estudos (CEBI, 2015).

O intervalo de confiança define os limites inferior e superior de um conjunto de valores que tem certa probabilidade de conter o valor verdadeiro do efeito da intervenção. Desta forma, o IC 95\% tem 95\% de probabilidade de incluir o valor real da eficácia da intervenção em análise. O uso do intervalo de confiança permite a interpretação do achado (se é estatisticamente significativo para um dado nível de significância ou não). Quando o intervalo de confiança contiver o valor nulo de efeito (linha vertical do gráfico, valor zero), o resultado não é considerado significativo estatisticamente) (Coutinho e Cunha, 2005).

O diamante (prisma) localizado na base do plot representa o tamanho médio do efeito entre os estudos envolvidos. Os seus vértices, esquerdo e direito, são representação dos limites do IC 95\% e seus vértices superior e inferior determinam o tamanho médio do efeito (projeção na linha graduada horizontal). Considera-se um resultado significativo estatisticamente, a presença completa do diamante em um dos lados do gráfico, sem que haja contato com a linha de valor zero) (CEBI, 2015).

Os riscos de vieses entre os estudos, notadamente aqueles de publicação, e a heterogeneidade entre eles, são avaliados graficamente por um outro tipo de diagrama, denominado funnel plot. 
O funnel plot, ou gráfico em funil, é um gráfico de dispersão em forma de triângulo elaborado com as estimativas de efeito dos estudos individuais (DR, representado pela projeção vertical do círculo no eixo das abscissas) e com os valores de desvio padrão no eixo das ordenadas (denominado standard error, observado nos gráficos com a sigla, em inglês, SE). Esta última medida representa o desvio padrão da distribuição das amostras populacionais dos estudos envolvidos. Salienta-se que a escala numérica no eixo das ordenadas é invertida, de modo a posicionar os estudos maiores e mais significativos em direção ao topo.

Um triângulo simétrico determinado por um vértice superior (centrado na estimativa de efeito médio dos estudos) e vértices laterais (determinam uma área em que estarão 95\% dos estudos se não houver vieses) é representado concomitantemente com a distribuição dos estudos. $\mathrm{Na}$ ausência de vieses e heterogeneidade entre as pesquisas, a distribuição dos círculos relativos aos estudos se dará dentro da área do triângulo (Sterne et al., 2011).

As informações sobre as estimativas de efeitos e intervalos de confiança para cada estudo são mostradas graficamente abaixo. As sínteses numéricas grupo-específicas, tamanhos de efeito, intervalos de confiança e os pesos dos estudos analisados, em porcentagem, também são evidenciados, como já descrito anteriormente, nos gráficos seguintes (forest e funnel plots).

Os dados relativos à sobrevida global de três, cinco e 10 anos são estudados abaixo. 
As informações referentes à sobrevida global de três anos estavam disponíveis em seis estudos, totalizando e reportando dados de 1197 pacientes, como demonstrado no Gráfico 1. Na análise conjunta, não houve diferenças estatisticamente significativas nos índices de sobrevida global de três anos após os tratamentos endoscópico e cirúrgico do câncer gástrico precoce (diferença de risco $=0,01$, intervalo de confiança de 95\% $=-0,02$ a 0,05). Os dados de RA dos efeitos médios dos estudos envolvidos foram 92,1\% (525/570) para a endoscopia e 92,02\% (577/627) para a cirurgia. A heterogeneidade foi classificada em baixa $\left(I^{2}=31 \%\right)$ e não se observou a presença de outliers (estudos aberrantes ou atípicos, inconsistentes, geralmente localizados fora do triângulo funnel), como comprovado no Gráfico 2.

Gráfico 1 - Sobrevida de três anos após os tratamentos endoscópico e cirúrgico do câncer gástrico precoce - Gráfico em floresta

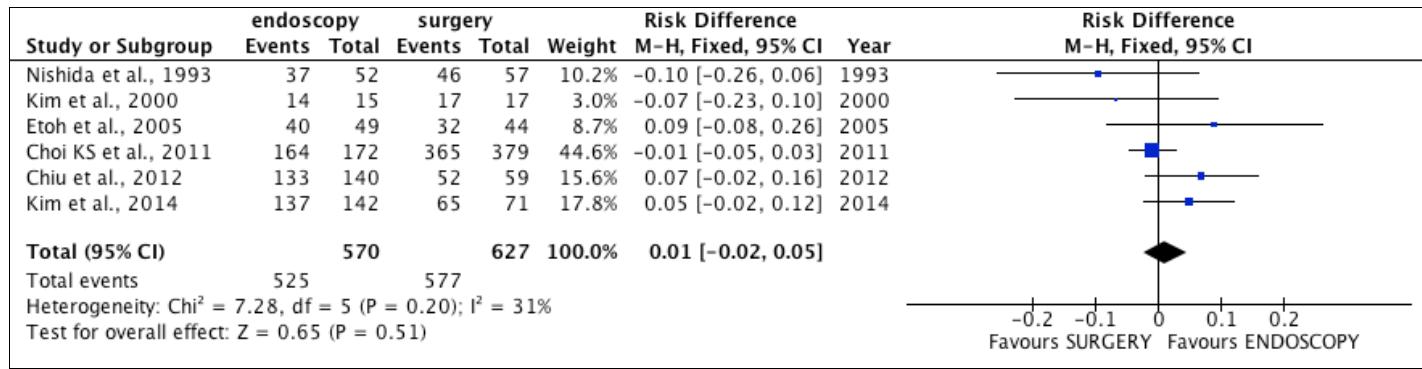


Gráfico 2 - Distribuição dos seis estudos que relatam a sobrevida de 3 anos após os tratamentos endoscópico e cirúrgico do câncer gástrico precoce - Gráfico em funil

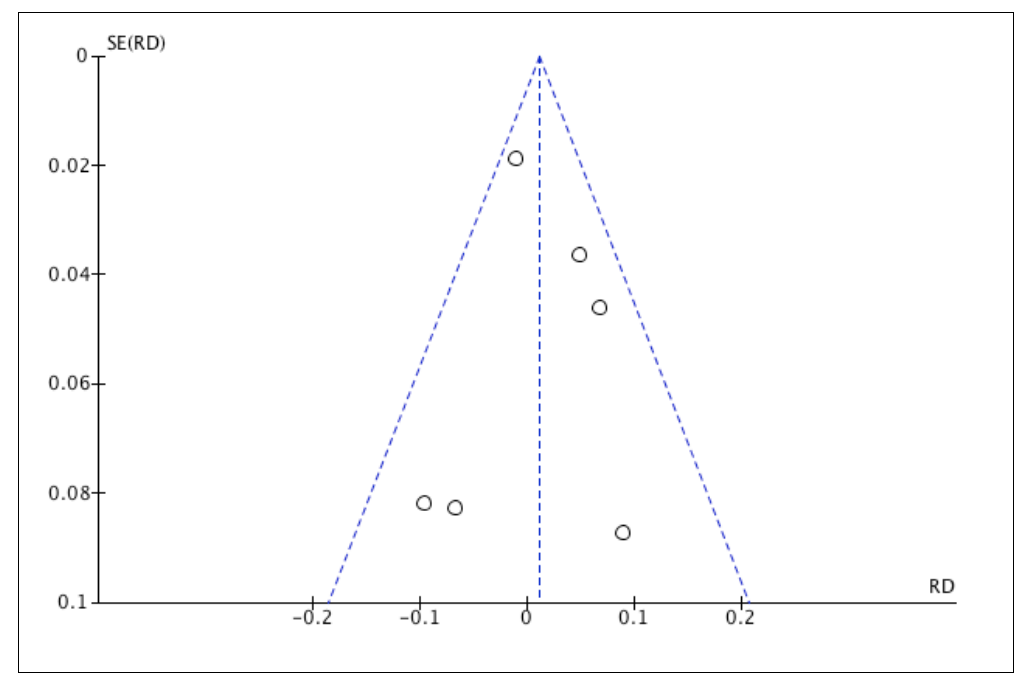

As informações de sobrevida de cinco anos foram obtidas de nove estudos. Entretanto, significativa heterogeneidade foi detectada $\left(x^{2}=20,87\right.$ e $\mathrm{I}^{2}=62 \%$ ) quando analisado este desfecho clínico (Gráfico 3). A análise de sensibilidade, realizada pela interpretação do funnel plot, identificou um trabalho discrepante (outlier, representado pela seta cinza) dos demais (Fukase et al., 1994), como verificado no Gráfico 4.

Gráfico 3 - Sobrevida de cinco anos após os tratamentos endoscópico e cirúrgico do câncer gástrico precoce - Gráfico em floresta

\begin{tabular}{|c|c|c|c|c|c|c|c|c|c|c|c|}
\hline \multirow[b]{2}{*}{ Study or Subgroup } & \multicolumn{2}{|c|}{ endoscopy } & \multicolumn{2}{|c|}{ surgery } & \multirow[b]{2}{*}{ Weight } & \multicolumn{2}{|l|}{ Risk Difference } & \multirow{2}{*}{\multicolumn{4}{|c|}{$\begin{array}{c}\text { Risk Difference } \\
\text { M-H, Fixed, } 95 \% \mathrm{Cl}\end{array}$}} \\
\hline & Events & Total & Events & Total & & M-H, Fixed, $95 \% \mathrm{Cl}$ & Year & & & & \\
\hline Nishida et al., 1993 & 32 & 52 & 41 & 57 & $4.8 \%$ & $-0.10[-0.28,0.07]$ & 1993 & & & & \\
\hline Fukase et al., 1994 & 100 & 116 & 59 & 59 & $6.9 \%$ & $-0.14[-0.21,-0.07]$ & 1994 & & & & \\
\hline Etoh et al., 2005 & 31 & 49 & 24 & 44 & $4.1 \%$ & $0.09[-0.11,0.29]$ & 2005 & & & & \\
\hline Choi KS et al., 2011 & 161 & 172 & 357 & 379 & $20.9 \%$ & $-0.01[-0.05,0.04]$ & 2011 & & & & \\
\hline Fukunaga et al., 2012 & 151 & 167 & 107 & 120 & $12.3 \%$ & $0.01[-0.06,0.08]$ & 2012 & & & & \\
\hline Park et al., 2014 & 105 & 108 & 112 & 117 & $9.9 \%$ & $0.01[-0.03,0.06]$ & 2014 & & & - & \\
\hline Kim et al., 2014 & 136 & 142 & 65 & 71 & $8.4 \%$ & $0.04[-0.03,0.11]$ & 2014 & & & & \\
\hline Choi et al., 2015 & 250 & 261 & 107 & 114 & $14.0 \%$ & $0.02[-0.03,0.07]$ & 2015 & & & - & \\
\hline Kim et al., 2015 & 161 & 165 & 283 & 292 & $18.6 \%$ & $0.01[-0.02,0.04]$ & 2015 & & & & \\
\hline Total $(95 \% \mathrm{CI})$ & & 1232 & & 1253 & $100.0 \%$ & $-0.00[-0.02,0.02]$ & & & & & \\
\hline Total events & 1127 & & 1155 & & & & & & & & \\
\hline $\begin{array}{l}\text { Heterogeneity: } \mathrm{Chi}^{2}= \\
\text { Test for overall effect: }\end{array}$ & $\begin{array}{l}0.87, \mathrm{df}= \\
=0.15(\end{array}$ & $\begin{array}{l}=8(P= \\
P=0.8\end{array}$ & $\begin{array}{l}=0.007) \\
88)\end{array}$ & $; 1^{2}=$ & & & & -0.5 & $\begin{array}{l}-0.25 \\
\text { Favours SURGERY }\end{array}$ & ${ }^{0}$ Favours E & $\begin{array}{cc}0.25 & 0.5 \\
\text { ENDOSCOPY } & \end{array}$ \\
\hline
\end{tabular}


Gráfico 4- Distribuição dos nove estudos que relatam a sobrevida de cinco anos após os tratamentos endoscópico e cirúrgico do câncer gástrico precoce - Gráfico em funil

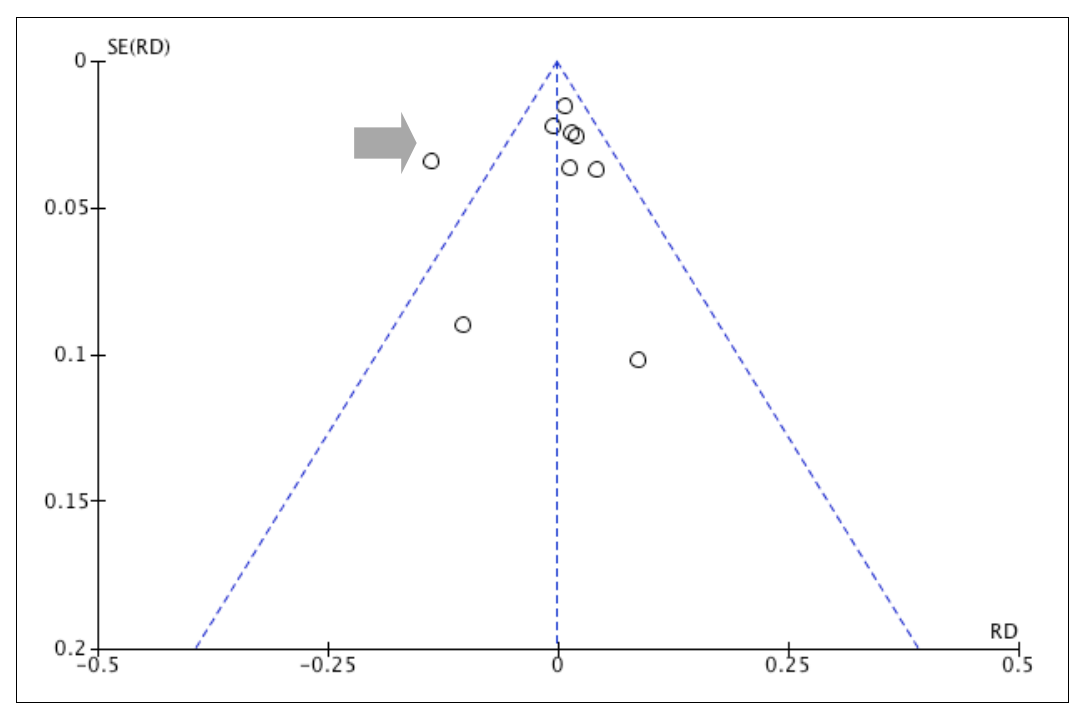

A exclusão deste artigo diminuiu a heterogeneidade para 0\% (Gráficos 5 e 6) e não alterou o achado de ausência de diferença estatística nas taxas de sobrevida de cinco anos entre os tratamentos endoscópico e cirúrgico do CGP (DR $=0,01$, IC 95\% = -0,01 a 0,03). Os dados obtidos baseiam-se na análise de 2310 pacientes. Os dados de RA dos efeitos médios dos estudos envolvidos foram 92,02\% (1027/1116) no braço da endoscopia e 91,8\% (1096/1194) no grupo da cirurgia. 
Gráfico 5 - Sobrevida de cinco anos após os tratamentos endoscópico e cirúrgico do câncer gástrico precoce (excluindo-se o outlier) - Gráfico em floresta

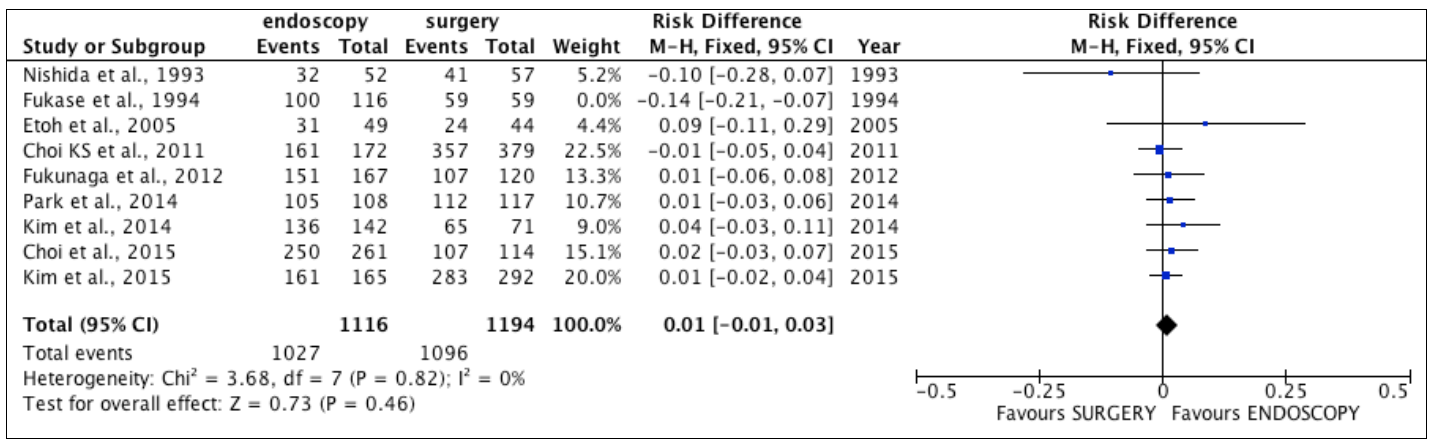

Gráfico 6 - Distribuição dos oito estudos que relatam a sobrevida de cinco anos após os tratamentos endoscópico e cirúrgico do câncer gástrico precoce (excluindo-se o outlier) - Gráfico em funil

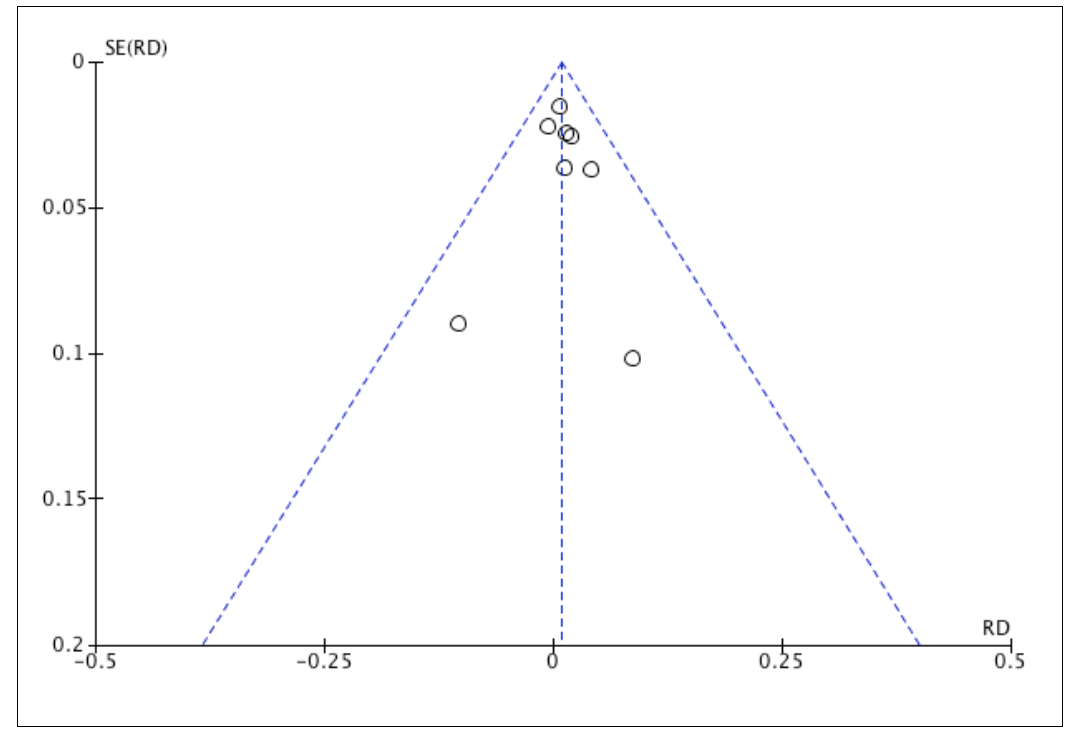

Numa análise de subgrupo, considerando os seis estudos disponíveis comparando ESD versus cirurgia, quatro coortes (1014 pacientes) avaliaram a sobrevida de cinco anos. A heterogeneidade foi $I^{2}=0 \%$. Não houve evidência de diferença entre as duas formas de abordagem (endoscópica e cirúrgica), observando-se DR $=0,02$, IC 95\% $=-0,01$ a 0,05), como demonstrado nos 
Gráficos 7 e 8. Os dados de RA dos efeitos dos estudos envolvidos foram 94,4\% (559/592) no grupo da ESD e 92,6\% (391/422) na cirurgia.

Gráfico 7 - Sobrevida de cinco anos após os tratamentos endoscópico (ESD) e cirúrgico do câncer gástrico precoce - Gráfico em floresta

\begin{tabular}{|c|c|c|c|c|c|c|c|c|}
\hline \multirow[b]{2}{*}{ Study or Subgroup } & \multicolumn{2}{|l|}{ ESD } & \multicolumn{2}{|c|}{ SURGERY } & \multicolumn{3}{|c|}{ Risk Difference } & \multirow{2}{*}{$\begin{array}{c}\text { Risk Difference } \\
\text { M-H, Fixed, } 95 \% \mathrm{Cl}\end{array}$} \\
\hline & Events & Total & Events & Total & Weight & M-H, Fixed, $95 \% \mathrm{Cl}$ & Year & \\
\hline Fukunaga et al., 2012 & 151 & 167 & 107 & 120 & $28.8 \%$ & $0.01[-0.06,0.08]$ & 2012 & \\
\hline Park et al., 2014 & 105 & 108 & 112 & 117 & $23.2 \%$ & $0.01[-0.03,0.06]$ & 2014 & \\
\hline Kim et al., 2014 & 136 & 142 & 65 & 71 & $19.5 \%$ & $0.04[-0.03,0.11]$ & 2014 & \\
\hline Choi et al., 2015 & 167 & 175 & 107 & 114 & $28.5 \%$ & $0.02[-0.04,0.07]$ & 2015 & \\
\hline Total $(95 \% \mathrm{CI})$ & & 592 & & 422 & $100.0 \%$ & $0.02[-0.01,0.05]$ & & \\
\hline Total events & 559 & & 391 & & & & & \\
\hline \multicolumn{8}{|c|}{$\begin{array}{l}\text { Heterogeneity: } \mathrm{Chi}^{2}=0.47, \mathrm{df}=3(P=0.93) ; I^{2}=0 \% \\
\text { Test for overall effect: } Z=1.24(P=0.22)\end{array}$} & 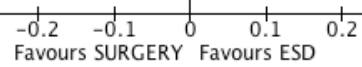 \\
\hline
\end{tabular}

Gráfico 8 - Distribuição dos quatro estudos que relatam a sobrevida de cinco anos após os tratamentos endoscópico (ESD) e cirúrgico do câncer gástrico precoce - Gráfico em funil

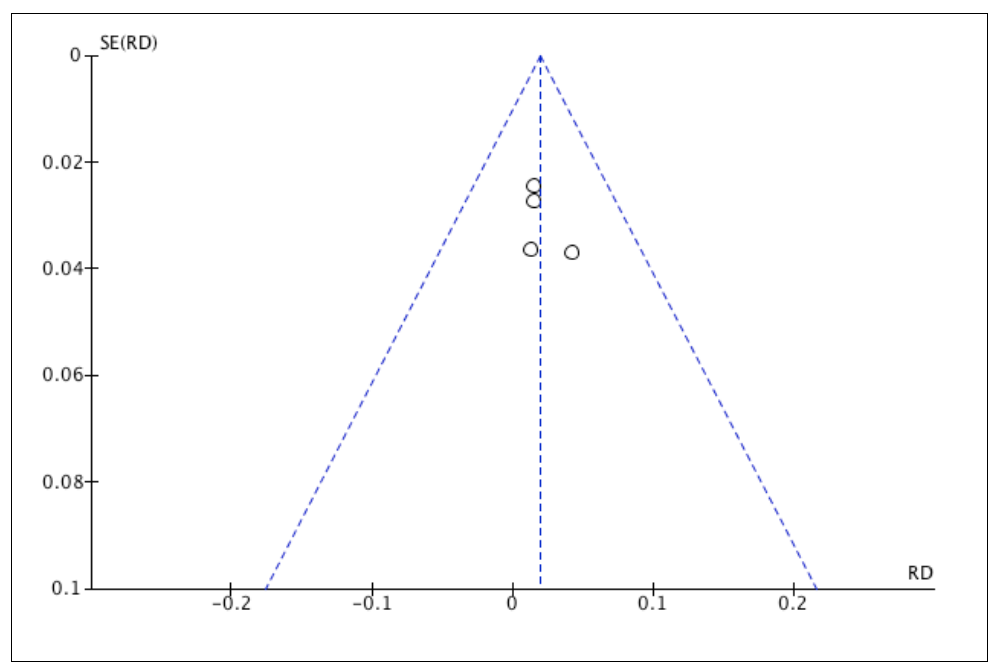

Os dados acerca da sobrevida de 10 anos estavam disponíveis em dois estudos. Uma significante heterogeneidade foi reconhecida $\left(x^{2}=27,58\right.$ e $\mathrm{I}^{2}=96 \%$ ), como verificado no Gráfico 9. Considerando o peso percentual de 24,8\% nesta análise, e sendo o outlier mais distante do triângulo (funil), representado pela seta cinza, o estudo publicado por Fukase et al. (1994) foi removido (Gráfico 10). 
Gráfico 9 - Sobrevida de 10 anos após os tratamentos endoscópico e cirúrgico do câncer gástrico precoce - Gráfico em floresta

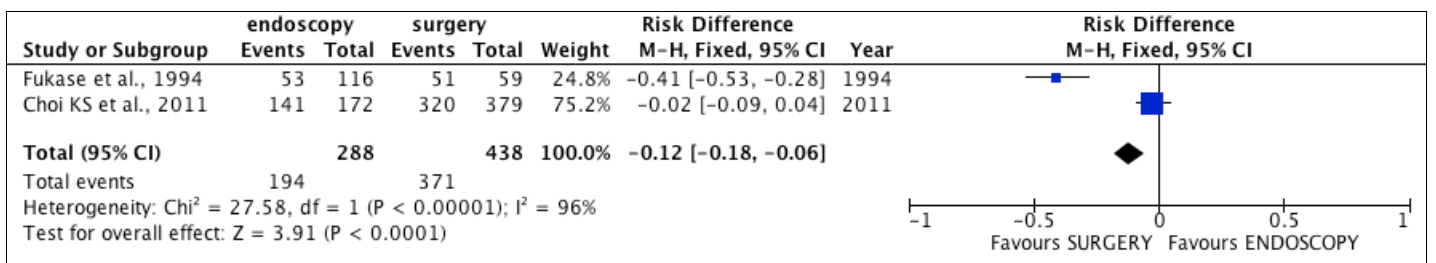

Gráfico 10 - Distribuição dos dois estudos que relatam a sobrevida de 10 anos após os tratamentos endoscópico e cirúrgico do câncer gástrico precoce - Gráfico em funil

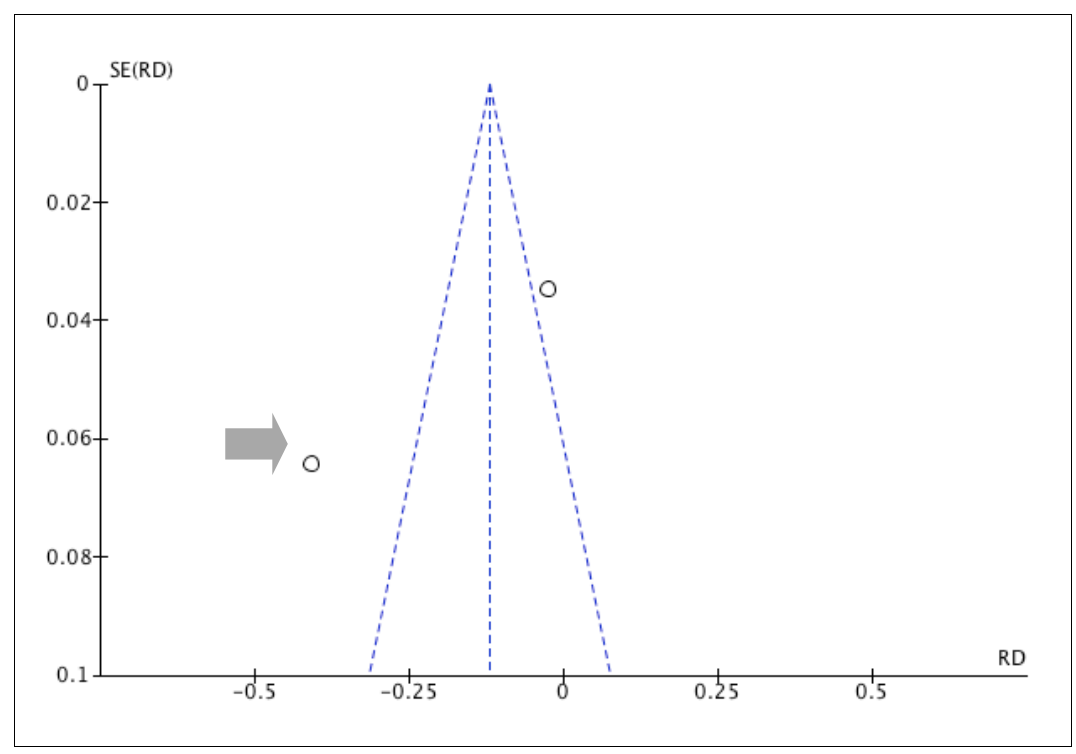

A análise dos dados do estudo residual (Choi KS et al., 2011) não demonstrou, em 551 pacientes, evidência de diferença nas taxas de sobrevida de 10 anos entre as abordagens endoscópica e cirúrgica para o tratamento do CGP (DR = -0,02 e IC 95\% = -0,15 a 0,10), de acordo com o Gráfico 11. Os RA dos efeitos médios do estudo analisado foram $81,9 \%$ (141/172) nos pacientes submetidos à endoscopia e 84,4\% (320/379) nos submetidos à cirurgia. 


\section{Gráfico 11 - Sobrevida de 10 anos após os tratamentos endoscópico e cirúrgico do câncer gástrico precoce (excluindo-se o outlier) - Gráfico em floresta}

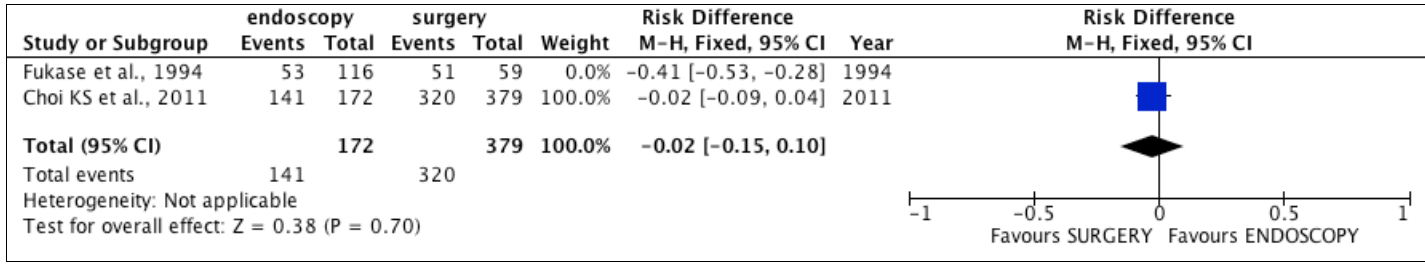

As morbidades relacionadas às diferentes formas de tratamento são consideradas e ponderadas abaixo. Essas complicações incluem quaisquer implicações adversas relacionadas ao procedimento, como previamente descrito, como fístula anastomótica, estenose, perfuração, hemorragia, infecção, íleo adinâmico ou mecânico, herniação, outros tipos de acometimento visceral ou, até mesmo, descompensação de doença clínica pré-existente (insuficiência renal aguda, infecção pulmonar, infarto agudo do miocárdio, e assim por diante).

Dados sobre complicações nos tratamentos endoscópico e cirúrgico do CGP foram identificados em oito estudos (Gráfico 12). Analisando-se estes dados, uma alta heterogeneidade foi inicialmente detectada $\left(x^{2}=\right.$ 34,50 e $\left.\mathrm{I}^{2}=80 \%\right)$, um funnel plot foi confeccionado e dois outliers principais, representados pelas setas cinzas, detectados (Chiu et al., 2012 e Kim et al., 2014), como visualizado no Gráfico 13. Uma vez que, excluindo-se estes estudos, como evidenciado nos Gráficos 14 e 15, a heterogeneidade persistiu elevada $\left(X^{2}=9,95\right.$ e $\left.\mathrm{I}^{2}=50 \%\right)$ e não houve mudança na propensão do diamante à análise do forest plot, eles foram mantidos na metanálise.

Os mesmos achados foram obtidos na aplicação do modelo de efeito randômico. A interpretação das informações de 2439 indivíduos demonstrou 
diferença significativa nas taxas de complicação entre os braços de tratamento avaliados para o CGP (DR $=-0,08$, IC 95\% $=-0,10$ a $-0,05$ ), comprovando melhores resultados com a abordagem endoscópica, ou seja, evidenciando uma RRA com a endoscopia. Os dados de RA dos efeitos dos estudos envolvidos foram 5,9\% (73/1228) na abordagem endoscópica e 12,1\% (147/1211) na cirúrgica.

O valor global do NNT (1/[DR]) foi 12, expressando que, para cada doze pacientes endoscopicamente tratados, um indivíduo seria beneficiado em não ter quaisquer complicações (seria prejudicado se tratado com cirurgia).

Gráfico 12 - Complicações após os tratamentos endoscópico e cirúrgico do câncer gástrico precoce - Gráfico em floresta

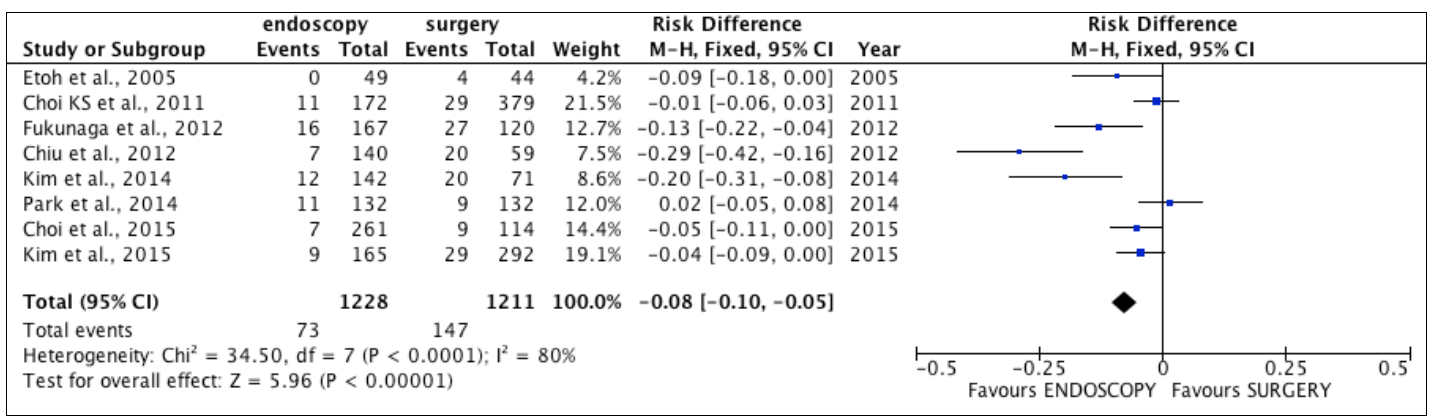


Gráfico 13 - Distribuição dos oito estudos que relatam as complicações após os tratamentos endoscópico e cirúrgico do câncer gástrico precoce - Gráfico em funil

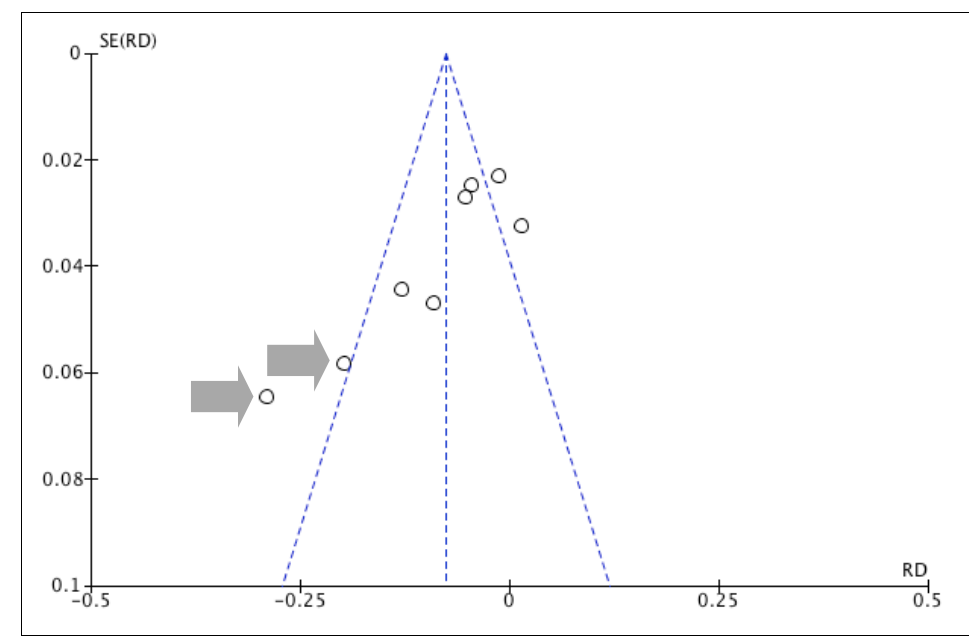
Gráfico 14 - Complicações após os tratamentos endoscópico e cirúrgico do câncer gástrico precoce (excluindo-se os outliers) - Gráfico em floresta

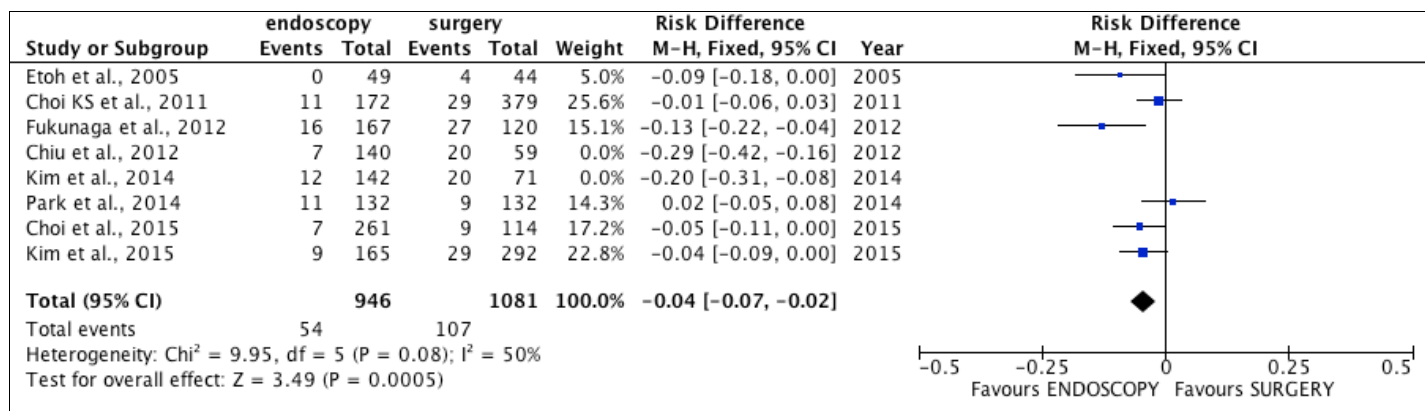




\section{Gráfico 15 - Distribuição dos seis estudos que relatam as complicações após os tratamentos endoscópico e cirúrgico do câncer gástrico precoce (excluindo-se os outliers) - Gráfico em funil}

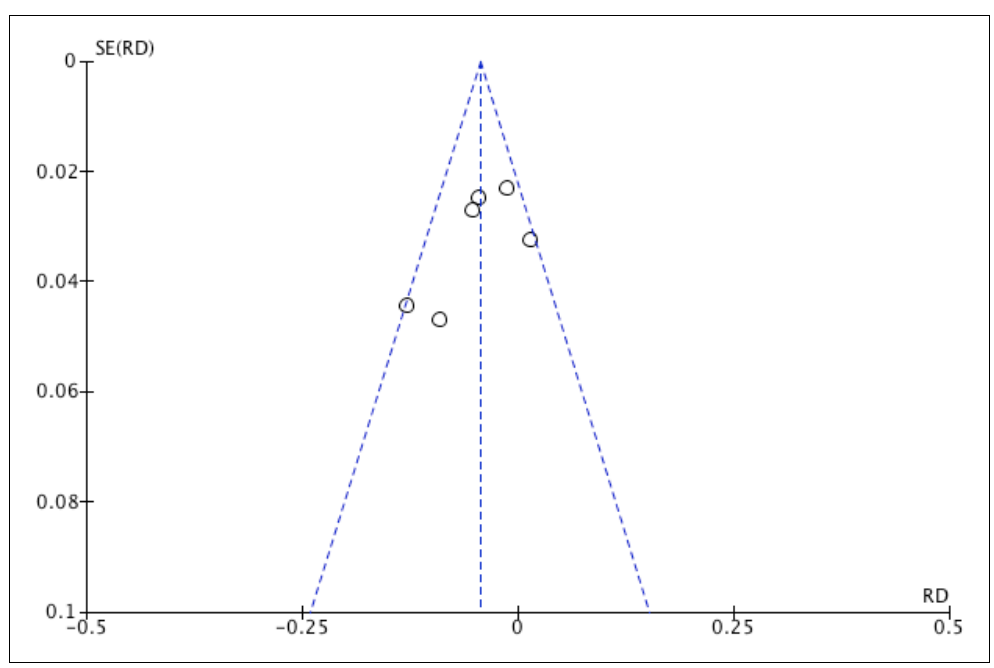

$\mathrm{Na}$ análise de subgrupos referentes ao ESD, cinco estudos (1252 pacientes) investigaram as informações relativas às complicações.

Uma alta heterogeneidade foi inicialmente constatada $\left(X^{2}=28,79\right.$ e $\mathrm{I}^{2}$ = 86\%), um funnel plot desenhado e dois outliers observados (Park et al., 2014 e Choi et al., 2015) (Gráficos 16 e 17). Visto que, excluindo-se estes trabalhos, a heterogeneidade permaneceu alta $\left(I^{2}=53 \%\right)$ (Gráficos 18 e 19) e não houve mudança na tendência do diamante do forest plot, eles foram mantidos para a metanálise. A apreciação dos dados mostrou diferença estatisticamente significativa (DR $=-0,12$, IC $95 \%=-0,15$ a $-0,08)$, favorecendo a abordagem endoscópica (Gráfico 16). Os dados de RA dos efeitos dos estudos envolvidos foram 6,3\% (48/756) no grupo da ESD e $17,1 \%$ (85/496) no da cirurgia, evidenciando uma RRA com a abordagem endoscópica. 
Gráfico 16 - Complicações após os tratamentos endoscópico (ESD) e cirúrgico do câncer gástrico precoce - Gráfico em floresta

\begin{tabular}{|c|c|c|c|c|c|c|c|c|c|c|}
\hline \multirow[b]{2}{*}{ Study or Subgroup } & \multicolumn{2}{|c|}{ ESD } & \multicolumn{2}{|c|}{ SURGERY } & \multirow[b]{2}{*}{ Weight } & Risk Difference & \multirow[b]{2}{*}{ Year } & \multirow{2}{*}{\multicolumn{3}{|c|}{$\begin{array}{c}\text { Risk Difference } \\
\text { M-H, Fixed, } 95 \% \mathrm{CI}\end{array}$}} \\
\hline & Events & Total & Events & Total & & M-H, Fixed, 95\% CI & & & & \\
\hline Chiu et al., 2012 & 7 & 140 & 20 & 59 & $14.1 \%$ & $-0.29[-0.42,-0.16]$ & 2012 & E & & \\
\hline Fukunaga et al., 2012 & 16 & 167 & 27 & 120 & $23.8 \%$ & $-0.13[-0.22,-0.04]$ & 2012 & $\longrightarrow$ & & \\
\hline Kim et al., 2014 & 12 & 142 & 20 & 71 & $16.1 \%$ & $-0.20[-0.31,-0.08]$ & 2014 & & & \\
\hline Park et al., 2014 & 11 & 132 & 9 & 132 & $22.5 \%$ & $0.02[-0.05,0.08]$ & 2014 & & & \\
\hline Choi et al., 2015 & 2 & 175 & 9 & 114 & $23.5 \%$ & $-0.07[-0.12,-0.02]$ & 2015 & $\rightarrow-$ & & \\
\hline Total $(95 \% \mathrm{Cl})$ & & 756 & & 496 & $100.0 \%$ & $-0.12[-0.15,-0.08]$ & & & & \\
\hline Total events & 48 & & 85 & & & & & & & \\
\hline $\begin{array}{l}\text { Heterogeneity: } \mathrm{Chi}^{2}=2 \\
\text { Test for overall effect: }\end{array}$ & $\begin{array}{l}3.79, \mathrm{df}= \\
=5.98(\end{array}$ & $\begin{array}{l}=4(P< \\
P<0.00\end{array}$ & $\begin{array}{l}0.0000 \\
0001)\end{array}$ & $1) ; 1^{2}=$ & $86 \%$ & & $\grave{-0.5}$ & $\begin{array}{c}-0.25 \\
\text { Favours ESD }\end{array}$ & \begin{tabular}{|cc}
0 & 0.25 \\
Favours & SURGERY
\end{tabular} & $\overrightarrow{0.5}$ \\
\hline
\end{tabular}

Gráfico 17 - Distribuição dos cinco estudos que relatam as complicações após os tratamentos endoscópico (ESD) e cirúrgico do câncer gástrico precoce - Gráfico em funil

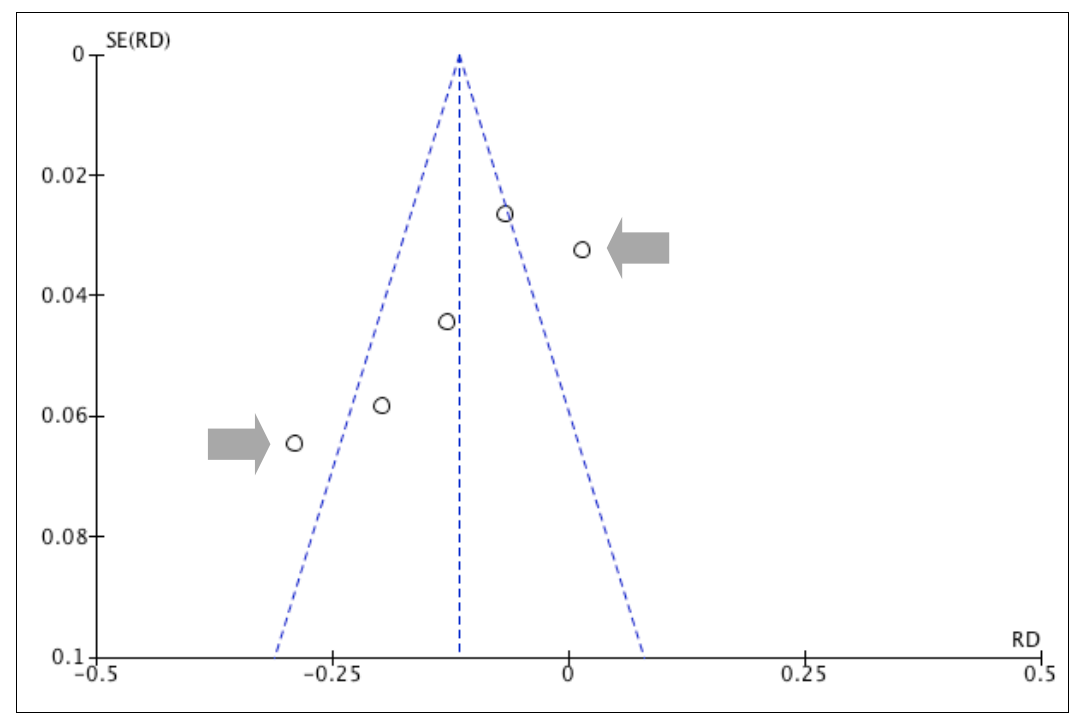

Gráfico 18 - Complicações após os tratamentos endoscópico (ESD) e cirúrgico do câncer gástrico precoce (excluindo-se os outliers) - Gráfico em floresta

\begin{tabular}{|c|c|c|c|c|c|c|c|c|c|}
\hline \multirow[b]{2}{*}{ Study or Subgroup } & \multicolumn{2}{|c|}{ ESD } & \multicolumn{2}{|c|}{ SURGERY } & \multirow[b]{2}{*}{ Weight } & Risk Difference & \multirow[b]{2}{*}{ Year } & \multirow{2}{*}{\multicolumn{2}{|c|}{$\begin{array}{c}\text { Risk Difference } \\
\text { M-H, Fixed, } 95 \% \mathrm{Cl}\end{array}$}} \\
\hline & Events & Total & Events & Total & & $\mathrm{M}-\mathrm{H}$, Fixed, $95 \% \mathrm{Cl}$ & & & \\
\hline Chiu et al., 2012 & 7 & 140 & 20 & 59 & $26.2 \%$ & $-0.29[-0.42,-0.16]$ & 2012 & $\longrightarrow$ & \\
\hline Fukunaga et al., 2012 & 16 & 167 & 27 & 120 & $44.0 \%$ & $-0.13[-0.22,-0.04]$ & 2012 & 上 & \\
\hline Kim et al., 2014 & 12 & 142 & 20 & 71 & $29.8 \%$ & $-0.20[-0.31,-0.08]$ & 2014 & & \\
\hline Park et al., 2014 & 11 & 132 & 9 & 132 & $0.0 \%$ & $0.02[-0.05,0.08]$ & 2014 & & \\
\hline Choi et al., 2015 & 2 & 175 & 9 & 114 & $0.0 \%$ & $-0.07[-0.12,-0.02]$ & 2015 & & \\
\hline Total $(95 \% \mathrm{CI})$ & & 449 & & 250 & $100.0 \%$ & $-0.19[-0.25,-0.13]$ & & & \\
\hline Total events & 35 & & 67 & & & & & & \\
\hline $\begin{array}{l}\text { Heterogeneity: } \mathrm{Chi}^{2}= \\
\text { Test for overall effect: }\end{array}$ & $\begin{array}{l}27, \mathrm{df}= \\
=6.15(\end{array}$ & $\begin{array}{l}2(P= \\
P<0.0\end{array}$ & $\begin{array}{l}0.12) ; 1^{2} \\
0001)\end{array}$ & $=53 \%$ & & & & $\begin{array}{l}-0.25 \\
\text { Favours ESD }\end{array}$ & ${ }^{0} \underset{1}{0.25}{ }_{\text {Favours SURGERY }}^{0.5}$ \\
\hline
\end{tabular}




\section{Gráfico 19 - Distribuição dos três estudos que relatam as complicações após os tratamentos endoscópico (ESD) e cirúrgico do câncer gástrico precoce (excluindo-se os outliers) - Gráfico em funil}

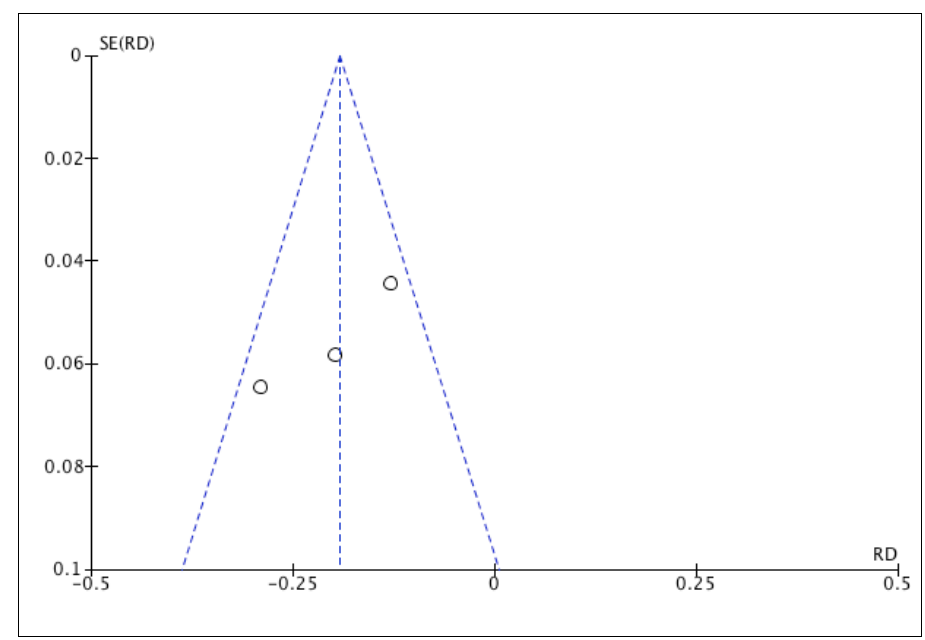

Ressecção completa, como já descrito, foi considerada a total excisão do câncer gástrico, definida como ausência de células neoplásicas viáveis no leito de procedimento.

Considerando-se as taxas de ressecção completa, comparando-se o tratamento endoscópico e cirúrgico do CGP, cinco artigos foram aproveitados. A heterogeneidade foi representada por $X^{2}=13,59$ e $\mathrm{I}^{2}=71 \%$ (Gráfico 20). O funnel plot evidenciou um outlier, representado pela seta cinza (Nishida et al., 1993), como mostrado no Gráfico 21. 
Gráfico 20 - Ressecções completas após os tratamentos endoscópico e cirúrgico do câncer gástrico precoce - Gráfico em floresta

\begin{tabular}{|c|c|c|c|c|c|c|c|c|c|c|}
\hline \multirow[b]{2}{*}{ Study or Subgroup } & \multicolumn{2}{|c|}{ endoscopy } & \multicolumn{2}{|c|}{ surgery } & \multirow[b]{2}{*}{ Weight } & \multicolumn{2}{|l|}{ Risk Difference } & \multirow{2}{*}{\multicolumn{3}{|c|}{$\begin{array}{c}\text { Risk Difference } \\
\mathrm{M}-\mathrm{H} \text {, Fixed, } 95 \% \mathrm{Cl}\end{array}$}} \\
\hline & Events & Total & Events & Total & & M-H, Fixed, $95 \% \mathrm{Cl}$ & Year & & & \\
\hline Nishida et al., 1993 & 40 & 55 & 57 & 57 & $18.6 \%$ & $-0.27[-0.39,-0.15]$ & 1993 & & $\Longrightarrow$ & \\
\hline Fukase et al., 1994 & 106 & 116 & 59 & 59 & $26.0 \%$ & $-0.09[-0.14,-0.03]$ & 1994 & & $\longrightarrow$ & \\
\hline Kim et al., 2000 & 17 & 20 & 35 & 35 & $8.5 \%$ & $-0.15[-0.31,0.01]$ & 2000 & & & \\
\hline Etoh et al., 2005 & 38 & 49 & 44 & 44 & $15.4 \%$ & $-0.22[-0.35,-0.10]$ & 2005 & & - & \\
\hline Kim et al., 2014 & 127 & 142 & 71 & 71 & $31.5 \%$ & $-0.11[-0.16,-0.05]$ & 2014 & & $\rightarrow-$ & \\
\hline Total $(95 \% \mathrm{CI})$ & & 382 & & 266 & $100.0 \%$ & $-0.15[-0.19,-0.11]$ & & & & \\
\hline Total events & 328 & & 266 & & & & & & & \\
\hline $\begin{array}{l}\text { Heterogeneity: } \mathrm{Chi}^{2}= \\
\text { Test for overall effect }\end{array}$ & $\begin{array}{l}13.59, d \\
Z=7.52\end{array}$ & $\begin{array}{l}f=4(P \\
2(P<0\end{array}$ & $\begin{array}{l}P=0.009 \\
.00001)\end{array}$ & 9); $I^{2}=$ & & & & -0.5 & $\begin{array}{c}-0.25 \\
\text { Favours SURGERY }\end{array}$ & 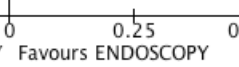 \\
\hline
\end{tabular}

Gráfico 21 - Distribuição dos cinco estudos que relatam as ressecções completas após os tratamentos endoscópico e cirúrgico do câncer gástrico precoce - Gráfico em funil

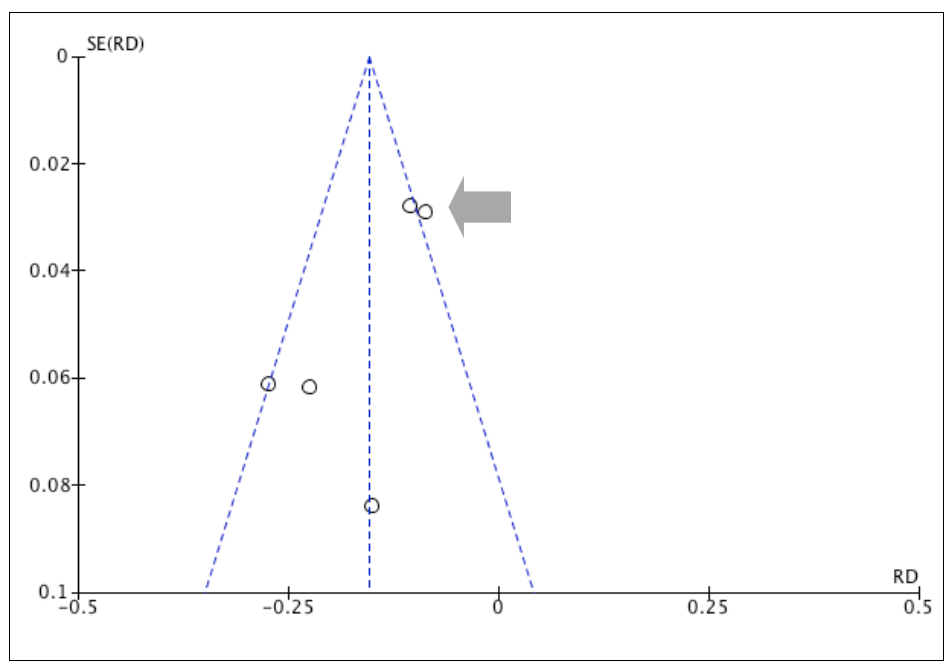

Excluindo-se este trabalho, a heterogeneidade diminuiu para $\mathrm{I}^{2}=41 \%$ e não houve evidência de modificação na tendência do diamante à interpretação do respectivo forest plot. A metanálise dos dados obtidos, assim, foi realizada considerando um total de 536 pacientes e quatro artigos. Ela constatou diferença significativa nas taxas de ressecção completa entre os tratamentos endoscópico e cirúrgico do CGP (DR = -0,13, IC 95\% = -0,17 a -0,09), comprovando melhores resultados no grupo da cirurgia (Gráficos 22 e 23). Os dados de RA dos efeitos dos estudos envolvidos foram $88,0 \%$ 
(288/327) nos pacientes tratados por endoscopia e 100\% (209/209) nos por cirurgia, observando-se um ARA com a abordagem cirúrgica.

A valor global do $\mathrm{NNH}(1 /[\mathrm{DR}])$ foi 7 , demonstrando que, para cada sete indivíduos que foram submetidos à abordagem endoscópica, um paciente seria beneficiado em não ter uma ressecção incompleta (teria sido tratado adequadamente se a abordagem cirúrgica tivesse sido escolhida).

Gráfico 22 - Ressecções completas após os tratamentos endoscópico e cirúrgico do câncer gástrico precoce (excluindo-se o outlier) - Gráfico em floresta

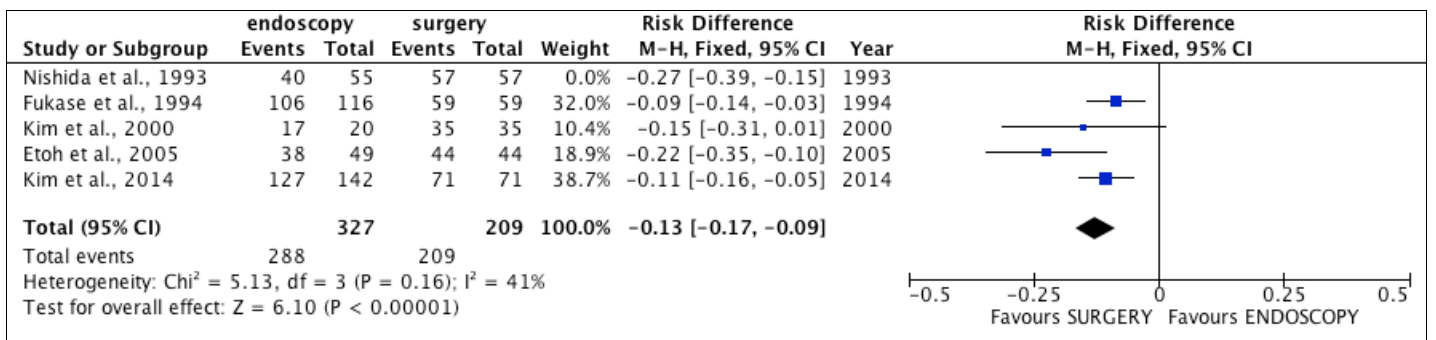

Gráfico 23 - Distribuição dos quatro estudos que relatam as ressecções completas após os tratamentos endoscópico e cirúrgico do câncer gástrico precoce (excluindo-se o outlier) - Gráfico em funil

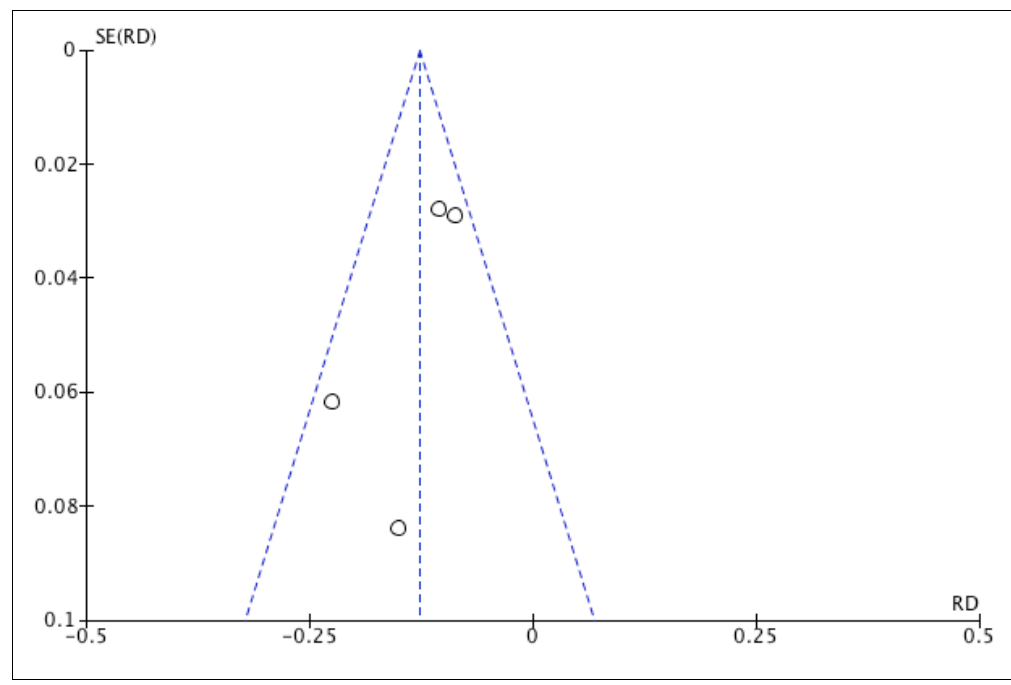


Recorrência foi definida como o reaparecimento, micro ou macroscópico, do câncer após o seu tratamento, e seguindo-se um período de não constatação clínica ou em exames subsidiários de imagem. Foi considerada, nesta análise, tanto a recorrência local como à distância. As informações quanto às taxas de recorrência obtidas são descritas a seguir.

Dados das taxas de recorrência foram identificados em sete estudos e um total de 2163 indivíduos (Gráfico 24). A heterogeneidade constatada foi elevada $\left(x^{2}=16,51\right.$ e $\left.I^{2}=64 \%\right)$, como verificado no respectivo funnel plot (Gráfico 25), que revelou dois outliers (Choi et al., 2015 e Kim et al., 2015).

Excluindo-se estes estudos da metanálise, a heterogeneidade diminuiu $\left(I^{2}=13 \%\right)$ e não houve mudança na tendência do diamante do forest plot correspondente.

Gráfico 24 - Recorrências após os tratamentos endoscópico e cirúrgico do câncer gástrico precoce - Gráfico em floresta

\begin{tabular}{|c|c|c|c|c|c|c|c|c|c|c|}
\hline \multirow[b]{2}{*}{ Study or Subgroup } & \multicolumn{2}{|c|}{ endoscopy } & \multicolumn{2}{|c|}{ surgery } & \multicolumn{3}{|c|}{ Risk Difference } & \multirow{2}{*}{\multicolumn{3}{|c|}{$\begin{array}{c}\text { Risk Difference } \\
\text { M-H, Fixed, } 95 \% \mathrm{Cl}\end{array}$}} \\
\hline & Events & Total & Events & Total & Weight & M-H, Fixed, $95 \% \mathrm{Cl}$ & Year & & & \\
\hline Kim et al., 2000 & 1 & 20 & 0 & 35 & $2.6 \%$ & $0.05[-0.07,0.17]$ & 2000 & & & \\
\hline Choi KS et al., 2011 & 2 & 172 & 4 & 379 & $24.2 \%$ & $0.00[-0.02,0.02]$ & 2011 & & - & \\
\hline Fukunaga et al., 2012 & 2 & 167 & 0 & 120 & $14.3 \%$ & $0.01[-0.01,0.03]$ & 2012 & & $t=$ & \\
\hline Park et al., 2014 & 0 & 108 & 0 & 117 & $11.5 \%$ & $0.00[-0.02,0.02]$ & 2014 & & - & \\
\hline Kim et al., 2014 & 5 & 142 & 0 & 71 & $9.7 \%$ & $0.04[-0.00,0.07]$ & 2014 & & 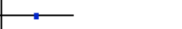 & \\
\hline Kim et al., 2015 & 8 & 165 & 1 & 292 & $21.6 \%$ & $0.05[0.01,0.08]$ & 2015 & & $\longrightarrow-$ & \\
\hline Choi et al., 2015 & 13 & 261 & 1 & 114 & $16.2 \%$ & $0.04[0.01,0.07]$ & 2015 & & $\longrightarrow$ & \\
\hline Total $(95 \% \mathrm{Cl})$ & & 1035 & & 1128 & $100.0 \%$ & $0.02[0.01,0.03]$ & & & $\bullet$ & \\
\hline \multirow{2}{*}{\multicolumn{8}{|c|}{$\begin{array}{l}\text { Heterogeneity: } \mathrm{Chi}^{2}=16.51, \mathrm{df}=6(\mathrm{P}=0.01) ; \mathrm{I}^{2}=64 \% \\
\text { Test for overall effect: } \mathrm{Z}=3.87(\mathrm{P}=0.0001)\end{array}$}} & & & \\
\hline & & & & & & & & $\begin{array}{lll}1 & 1 & 1 \\
-0.2 & -0.1 & 0 \\
\text { Favours ENDOSCOPY }\end{array}$ & $\stackrel{1}{1} \stackrel{1}{0.1}$ & 0.2 \\
\hline
\end{tabular}


Gráfico 25 - Distribuição dos sete estudos que relatam as recorrências após os tratamentos endoscópico e cirúrgico do câncer gástrico precoce - Gráfico em funil

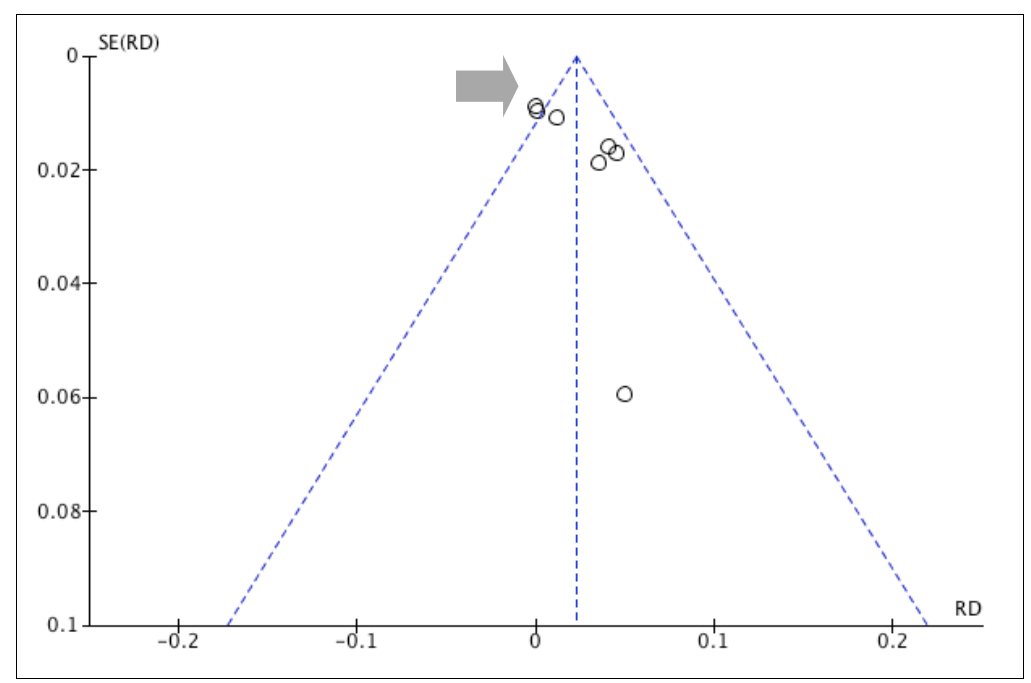

Um total de 1331 pacientes foram considerados. Não houve evidência de diferença quanto às taxas de recorrência entre as duas formas de tratamento do CGP (DR = 0,01, IC 95\% = -0,00 a 0,02) (Gráficos 26 e 27). Os dados de RA dos efeitos dos estudos envolvidos foram 1,6\% (10/609) na abordagem endoscópica e 0,5\% (4/722) na cirúrgica.

Gráfico 26 - Recorrências após os tratamentos endoscópico e cirúrgico do câncer gástrico precoce (excluindo-se os outliers) Gráfico em floresta

\begin{tabular}{|c|c|c|c|c|c|c|c|c|}
\hline \multirow[b]{2}{*}{ Study or Subgroup } & \multicolumn{2}{|c|}{ endoscopy } & \multicolumn{2}{|c|}{ surgery } & \multicolumn{3}{|c|}{ Risk Difference } & \multirow{2}{*}{$\begin{array}{c}\text { Risk Difference } \\
\mathrm{M}-\mathrm{H} \text {, Fixed, } 95 \% \mathrm{CI}\end{array}$} \\
\hline & Events & Total & Events & Total & Weight & M-H, Fixed, $95 \% \mathrm{CI}$ & Year & \\
\hline Kim et al., 2000 & 1 & 20 & 0 & 35 & $4.2 \%$ & $0.05[-0.07,0.17]$ & 2000 & \\
\hline Choi KS et al., 2011 & 2 & 172 & 4 & 379 & $38.9 \%$ & $0.00[-0.02,0.02]$ & 2011 & $\rightarrow$ \\
\hline Fukunaga et al., 2012 & 2 & 167 & 0 & 120 & $22.9 \%$ & $0.01[-0.01,0.03]$ & 2012 & T \\
\hline Park et al., 2014 & 0 & 108 & 0 & 117 & $18.5 \%$ & $0.00[-0.02,0.02]$ & 2014 & I \\
\hline Kim et al., 2014 & 5 & 142 & 0 & 71 & $15.6 \%$ & $0.04[-0.00,0.07]$ & 2014 & $\Longrightarrow$ \\
\hline Kim et al., 2015 & 8 & 165 & 1 & 292 & $0.0 \%$ & $0.05[0.01,0.08]$ & 2015 & \\
\hline Choi et al., 2015 & 13 & 261 & 1 & 114 & $0.0 \%$ & $0.04[0.01,0.07]$ & 2015 & \\
\hline Total $(95 \% \mathrm{Cl})$ & & 609 & & 722 & $100.0 \%$ & $0.01[-0.00,0.02]$ & & 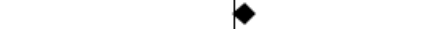 \\
\hline Total events & 10 & & 4 & & & & & \\
\hline $\begin{array}{l}\text { Heterogeneity: } \mathrm{Chi}^{2}=4 \\
\text { Test for overall effect: }\end{array}$ & $\begin{array}{l}61, \mathrm{df}= \\
=1.72\end{array}$ & $\begin{array}{l}4(P= \\
P=0.0\end{array}$ & $\begin{array}{l}0.33) ; 1^{2} \\
\text { 99) }\end{array}$ & $=13 \%$ & & & & $\begin{array}{ccccc} & 1 & 1 & 1 \\
-0.2 & -0.1 & 0 & 0.1 & 0.2 \\
\text { Favours ENDOSCOPY } & \text { Favours SURGERY }\end{array}$ \\
\hline
\end{tabular}




\section{Gráfico 27 - Distribuição dos cinco estudos que relatam as recorrências após os tratamentos endoscópico e cirúrgico do câncer gástrico precoce (excluindo-se os outliers) - Gráfico em funil}

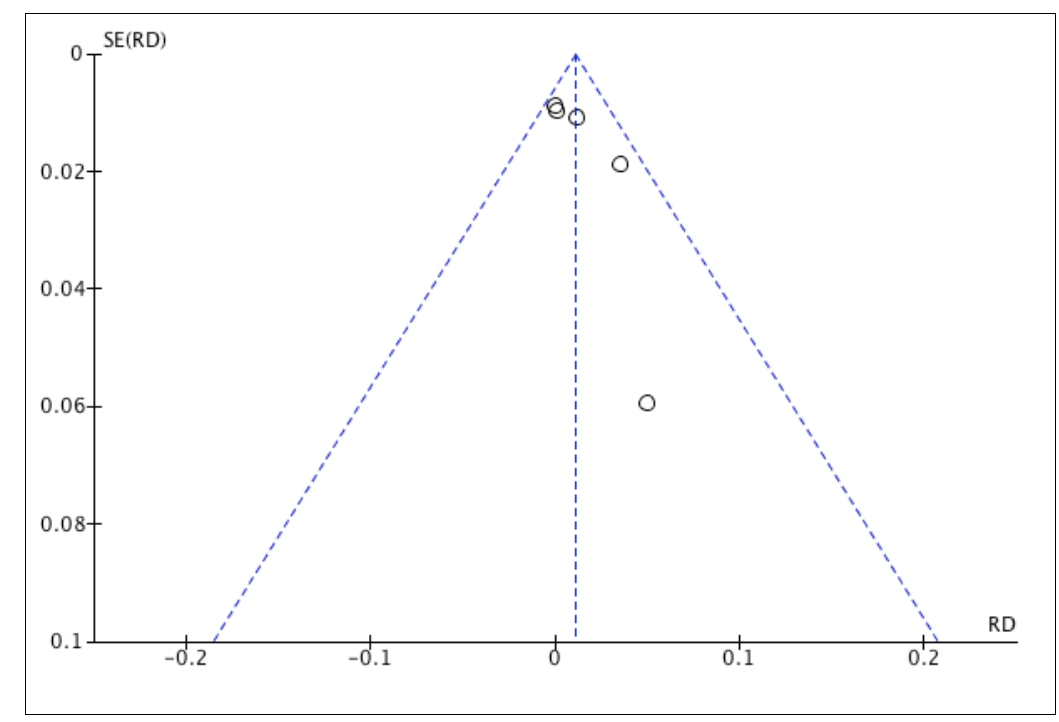

As informações sobre mortalidade embasam-se nas mortes em decorrência do procedimento, e incluem peritonite devido à perfuração do sítio de abordagem, estrangulamento de intestino delgado, sangramento de artéria esplênica e fístula de anastomose.

Os dados relativos à mortalidade estavam disponíveis em quatro estudos e um total de 1107 pacientes foram analisados (Gráfico 28). Não se detectou heterogeneidade $\left(x^{2}=0,85\right.$ e $\left.\mathrm{I}^{2}=0 \%\right)$, como observado no funnel plot acima (Gráfico 29).

Na metanálise, não se verificou diferença significativa de mortalidade entre os dois grupos comparados de tratamento do CGP (DR $=-0,01$, IC $95 \%=-0,02$ a 0,00$)$. Os dados de RA dos efeitos médios dos estudos envolvidos foram 0\% (0/493) na endoscopia e 0,6\% (4/614) na cirurgia. 
Gráfico 28 - Mortalidade após os tratamentos endoscópico e cirúrgico do câncer gástrico precoce - Gráfico em floresta

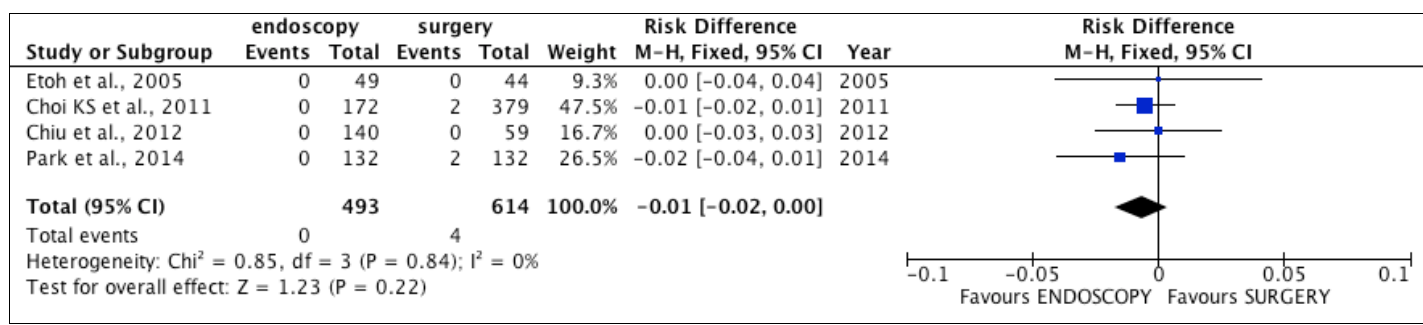

Gráfico 29 - Distribuição dos quatro estudos que relatam a mortalidade após os tratamentos endoscópico e cirúrgico do câncer gástrico precoce - Gráfico em funil

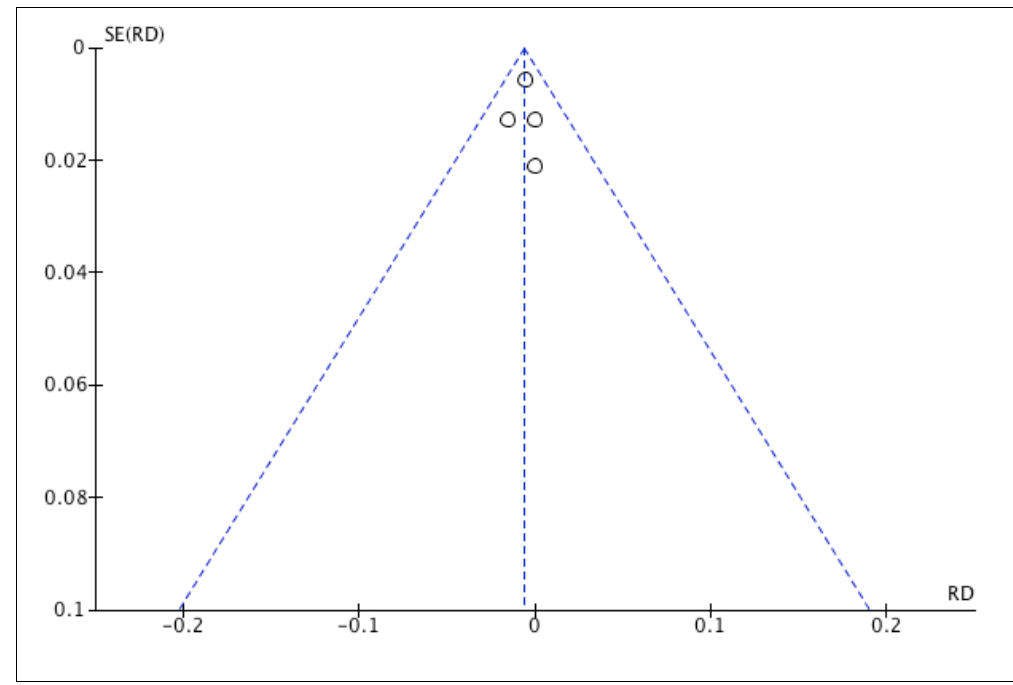




\section{DiscussÃo}


A gastrectomia, associada ou não à linfadenectomia, continua sendo o procedimento terapêutico oncológico convencional para tratamento dos pacientes portadores de câncer gástrico precoce (Gama-Rodrigues et al., 1986 e 1992). Entretanto, os métodos de ressecção endoscópica têm se tornado uma alternativa curativa menos invasiva para um grupo seleto de pacientes (Gotoda, 2007; JGCA, 2011b).

Basicamente, os dois principais métodos de abordagem endoscópica consistem em ressecção tumoral, seja ele realizado por mucosectomia ou dissecção submucosa endoscópica.

Muitos estudos têm demonstrado que o tratamento endoscópico do CGP é seguro, até mesmo para pacientes idosos (Hirasaki et al., 2005; Kakushima et al., 2007; Isomoto et al., 2010; Toyokawa et al., 2011; Tokioka et al., 2012). Atualmente, especialmente após 2004, a EMR foi paulatinamente substituída pela ESD, pois este apresenta maiores índices de ressecção en bloc e remoção endoscópica e patológica completa (Park et al., 2011). Mesmo desconsiderando os estudos mais antigos, publicados no início da década de 1990 por Nishida et al. (1993) e Fukase et al. (1994), quando os métodos de ressecção endoscópica ainda não eram totalmente e bem estabelecidos, as taxas de sobrevida de três e cinco anos, assim como os dados de ressecção completa, não mudaram significativamente. 
As taxas de ressecção en bloc da dissecção submucosa tendem a ser mais elevadas, uma vez que, lesões maiores que $2 \mathrm{~cm}$, não podem ser removidas adequadamente por mucosectomia, mesmo com técnica apurada, em virtude da limitação do método (Chaves et al., 2013). A ESD está associada a maiores taxas de ressecção en bloc que a EMR (92\% versus 52\%), assim como ressecções R0 (82\%-92\% versus 42\%-43\%) (ASGE, 2015).

Algumas metanálises demonstraram que a ESD está associada a menores taxas de recorrência que a EMR. Entretanto, ela tem algumas desvantagens, incluindo tempo de procedimento e taxas de complicação maiores (Cao et al., 2009; Lian et al., 2012; Facciorusso et al., 2014).

Esta revisão sistemática não foi capaz de comparar, numa análise de subgrupo e por indisponibilidade de dados, as taxas de ressecção completa da ESD versus cirurgia. Apesar de ser sabido que a ressecção R0 do CaG, ou seja, aquela em que não há células microscopicamente viáveis, estar próxima de $100 \%$ com a abordagem cirúrgica, nenhum estudo considerando esta comparação foi identificado. De acordo com Kim et al. (2014) e Choi et al. (2015), as taxas de ressecção completa, considerando a dissecção submucosa endoscópica, podem atingir 97,7\% em alguns casos selecionados. Neste estudo, os elevados índices de remoção tumoral completa resultaram, como observado no Gráfico 26, em menores valores de recorrência, semelhantes aos do grupo cirúrgico. É importante ressaltar que, mesmo em situações em que as margens de ressecção são positivas (no caso, as laterais ou radiais), um procedimento endoscópico complementar de re-ressecção é factível (Shimamura et al., 2013), habitualmente não comprometendo a sobrevida. 
Apesar dos critérios expandidos para a ressecção endoscópica, propostos por Gotoda (2007) estarem baseados na análise de peças cirúrgicas, habitualmente os médicos optam por uma modalidade de tratamento baseados em estadiamento pré-operatório com poucas ou limitadas informações, como os achados macroscópicos da endoscopia e os dados histopatológicos contidos nas biópsias das lesões (Park et al., 2014). Sabe-se que, em alguns casos, discrepâncias e não concordâncias, não desprezíveis, dos achados histológicos fornecidos pelas pinças de biópsia endoscópica e peças de ressecção cirúrgica, podem estar presentes (Lee et al., 2010), caracterizando um fator de confusão. Uma limitação importante dos estudos disponíveis é que os dados e informações obtidos incluem o relatório anatomopatológico final retrospectivo após o tratamento endoscópico ou cirúrgico do CGP, em vez de critérios definidos na avaliação pré-tratamento. De qualquer forma, todas as informações consideraram somente pacientes com CGP, adicionando, por outro lado, mais força à evidência encontrada. Como mencionado previamente, alguns estudos recentes têm constatado que os desfechos de longo prazo, após ESD para CGP do subtipo histológico indiferenciado, são excelentes (Abe et al., 2013; Oka et al., 2014).

Num estudo de pacientes que foram submetidos à gastrectomia radical para o tratamento de CGP, nenhum dos 1230 tumores intramucosos bem diferenciados menores que $3 \mathrm{~cm}$ de diâmetro tinha metástase linfonodal associada, independentemente da presença de ulceração (Gotoda et al., 2000). Considerando-se a presença de ulceração em todos os pacientes com diagnóstico de CGP, a probabilidade de envolvimento linfonodal 
metastático aumenta significativamente para os CGP ulcerados $(3,4 \%)$ comparada aos CGP não ulcerados (0,5\%) (Gotoda et al., 2000). Numa análise de subgrupo, foi demonstrado que os tumores confinados à mucosa, sem ulceração, não têm linfonodos acometidos, independentemente do seu tamanho (IC 95\%, 0 a 0,4\%). Tumores intramucosos com ulceração, e menores que $30 \mathrm{~mm}$, mostraram-se livres de positividade linfonodal (IC 95\%, 0 a 0,3\%) (Gotoda et al., 2000; Lee et al., 2011).

Um estudo comparativo e prospectivo foi realizado no Japão, envolvendo os desfechos clínicos de pacientes submetidos à ressecção endoscópica (EMR e ESD) indicadas pelos critérios absolutos e expandidos (Yamaguchi et al., 2009; Lee et al., 2011). O total de 589 CGP foram divididos em dois braços, considerando os critérios estabelecidos. Ressecções en bloc, completas e curativas foram alcançadas em 98,6 e 93, 95,1 e 88,5 , e 97,1 e 91,1\% das lesões precoces dos grupos de indicação absoluta e expandida, respectivamente. A sobrevida global foi igualmente adequada, do ponto de vista estatístico, nos dois braços analisados, e a sobrevida específica para a doença foi 100\% (Yamaguchi et al., 2009; Lee et al., 2011).

Uma metanálise avaliando os desfechos da ESD no tratamento do CGP, considerando os critérios de indicação para a ressecção endoscópica, demonstrou que os resultados são semelhantes nos grupos que preenchiam os critérios absolutos e expandidos (Peng et al., 2015).

Uma vez que estudos recentes indicam resultados comparáveis entre a cirurgia e a ressecção endoscópica de pacientes com CGP preenchendo os critérios absolutos e expandidos de Gotoda (2007), e considerando todas as variáveis envolvidas na decisão da melhor conduta (listadas abaixo), uma 
posição menos prejudicial e invasiva deveria ser inicialmente considerada e, se possível, adotada. Assim, uma abordagem cautelosa e cuidadosa de indivíduos satisfazendo os critérios expandidos deveria ser escolhida, uma vez que um número limitado de estudos, reportando desfechos equivalentes aos de grupos cirúrgicos, estão publicados (Chung et al., 2014; Fukunaga et al., 2016). Maior número de ressecções endoscópicas inadequadas ou recorrência seriam fatores relacionados a essa prática clínica. Para aumentar a segurança desta afirmação, ensaios clínicos randomizados e prospectivos, bem desenhados, são necessários para avaliar a possível superioridade da ressecção endoscópica comparada à cirurgia, considerando-se as indicações expandidas.

A definição de qual modalidade de tratamento é mais adequada compreende uma complexa interação de fatores, como as condições clínicas e idade do indivíduo, características do tumor, conhecimentos e habilidades técnicas dos profissionais responsáveis (cirurgião, patologista e endoscopista), além da infraestrutura hospitalar.

\subsection{Síntese das Evidências}

De modo geral, a evidência referente às taxas de sobrevida parece ser suficiente para determinar a eficácia comparativa das ressecções endoscópicas sobre os procedimentos cirúrgicos, uma vez que nenhuma diferença estatisticamente importante foi verificada.

Mesmo no início da década de 1990, Nishida et al. (1993), analisando 52 pacientes idosos com mais de 75 anos de idade que foram submetidos à abordagem endoscópica para o CGP, incluindo a mucosectomia associada 
ou não ao laser ou picibanil (agente esclerosante OK-432), demonstraram curvas de sobrevida similares comparada a 57 indivíduos do grupo cirúrgico. Interessante notar que uma das indicações para a preferência pelo tratamento endoscópico, neste artigo, eram as condições clínicas préoperatórias inadequadas.

Analisando-se os nove estudos envolvendo a sobrevida de 5 anos, somente um estudo (Fukase et al., 1994) foi discrepante dos demais, mostrando uma significativa redução do risco absoluto de 13,8\% quando o acesso endoscópico era considerado $(\mathrm{NNH}=7)$. Este estudo foi removido da análise geral (outlier). Na análise de subgrupo, considerando as quatro pesquisas disponíveis comparando ESD versus cirurgia (1014 pacientes), nenhuma diferença foi observada. Naquele tempo, a modalidade de tratamento endoscópico compreendia outras alternativas, como a cauterização com corrente de alta frequência e irradiação com YAG-laser, não utilizada nos dias atuais. Os autores relatam mais mortes, no período de cinco anos, no grupo do tratamento endoscópico, justificadas simplesmente por ter havido mais complicações graves neste braço do estudo.

Outros trabalhos têm demonstrado que o tratamento endoscópico do CGP pode alcançar curvas de sobrevida semelhantes àquelas vistas em pacientes submetidos à gastrectomia, independentemente do critério de ressecção adotado, se absoluto ou expandido (Fukunaga et al., 2012; Park et al., 2014; Kim et al., 2014). Entretanto, ensaios clínicos randomizados, controlados e prospectivos devem ser realizados para melhor consolidar apropriadamente esta informação e determinar a melhor opção terapêutica. 
Evidência aceitável foi apontada em relação às taxas de complicação. A análise dos dados de 2439 pacientes comprovou diferença significativa considerando-se este desfecho clínico nos pacientes submetidos aos tratamentos endoscópico e cirúrgico do CGP (DR = -0,08, IC 95\% = -0,10 a 0,05 e $\mathrm{P}<$ 0,00001), favorecendo a endoscopia. O NNT global foi 12, demonstrando que, para cada doze indivíduos endoscopicamente tratados, um seria beneficiado em não ter quaisquer tipos de complicação (de outro modo, seria prejudicado caso fosse submetido a tratamento cirúrgico). As taxas de complicação relacionadas ao tratamento endoscópico e cirúrgico, nesta metanálise, foram 5,9\% (73/1228) e 12,1\% (147/1211), respectivamente. A abordagem cirúrgica estava associada, mais frequentemente, a complicações maiores, como sangramento, obstrução intestinal, fistulas, estenoses anastomóticas, isquemia ou perfuração de vísceras ocas e aderências pósoperatórias, que necessitaram intervenção subsequente, fosse por endoscopia ou reoperação, demandando aumento do tempo de hospitalização. Na maioria dos casos, os eventos adversos relacionados à endoscopia, são sangramento e perfuração. As complicações imediatas e tardias do grupo submetido à ressecção endoscópica incluíam sangramento e perfuração, comumente relatados na literatura com taxas de 4,3\% e 5,3\%, respectivamente (Oda et al., 2013; Saito et al., 2014; Kosaka et al., 2014).

Uma vantagem cientificamente demonstrada é que, nestes casos, habitualmente os indivíduos são reabordados com sucesso por algum tipo de modalidade endoscópica, como por exemplo, a coagulação com plasma de argônio, a cauterização com a própria faca eletrocirúrgica (knife) ou a colocação de clipes metálicos. 
Considerando-se que os estudos selecionados foram desenvolvidos em centros de referência, altamente especializados, com grande volume de cirurgias gastrointestinais e unidades de endoscopia de excelência, a reprodutibilidade destas condutas e seus resultados devem ser interpretados com cautela.

No subgrupo ESD versus cirurgia (1252 pacientes), uma desigualdade estatisticamente relevante, favorecendo o tratamento endoscópico, também foi verificada.

As informações disponíveis, referentes às ressecções completas, confirmam as vantagens do procedimento cirúrgico em obter margens de ressecção livres, uma vez que ele permite, em geral, atingir limites mais distantes. Houve uma significativa diferença favorecendo a gastrectomia (DR = $13 \%$, IC $95 \%=-0,17$ a $-0,09, \mathrm{P}<0,00001, \mathrm{NNH}=7)$. Apesar de consideravelmente mais eficiente em se conseguir ressecções R0, a abordagem cirúrgica, como anteriormente citado, incorpora um risco de complicações, mesmo em mãos experientes. A análise, considerando o NNH, permite informar que, para cada sete indivíduos tratados por endoscopia, um teria o benefício de não ter uma ressecção incompleta. São relatadas na literatura menores taxas de ressecção completa do CGP quando procedida a mucosectomia (Kim et al., 2000; Etoh et al., 2005). Kim et al. (2000), realizando EMR com dispositivo de ligadura, relataram taxa de ressecção completa para o CGP de 85\%, mesmo se mais de uma sessão fosse necessária. Etoh et al. (2005), também executando mucosectomia, reportaram uma taxa de ressecção incompleta de 22,5\%. Ambos autores enfatizam a possibilidade de procedimentos de ressecção endoscópica sequenciais, principalmente nos 
casos de margens radiais acometidas ou, até mesmo, cirurgia de resgate, sem prejuízo nos índices de cura. Atualmente, com o advento e desenvolvimento técnico das ESDs, que possibilitam margens mais expandidas, os resultados tendem a mostrar progresso. A presença de fibrose submucosa tem sido associada a menores taxas de ressecção completa e maiores taxas de perfuração (Higashimaya et al., 2013).

Os dados de recorrência não demonstraram diferenças entre os dois braços de tratamento após a completa remoção do tumor, ou seja, ressecção $\mathrm{RO}(\mathrm{DR}=1 \%, \mathrm{IC} 95 \%=-0,00$ a 0,02, $\mathrm{P}=0,09)$. Como descrito anteriormente, o retratamento endoscópico ou cirurgia podem ser opções de reabordagem de acordo com o reestadiamento tumoral. Sabe-se que as modalidades de tratamento endoscópico são menos invasivas e preservam o estômago, o que pode proporcionar o aparecimento de outras lesões, sejam elas, recidivas ou nova neoplasia primária (metacronicidade). A maioria destas lesões podem ser diagnosticadas por endoscopias periódicas de vigilância e serem detectadas em estádios precoces.

Não houve evidência de diferença, considerando-se as informações sobre mortalidade, nos quatro estudos submetidos à metanálise (DR = 1\%, IC $95 \%=-0,02$ a $0,00, P=0,22)$. Apesar de não haver significância estatística, taxa de mortalidade maior foi observada no grupo cirúrgico. Nesta metanálise, a taxa de mortalidade no grupo submetido a tratamento endoscópico foi zero (0/493) e, no braço cirúrgico, 0,65\% (4/614). Apesar da cirurgia estar associada a taxas de complicação maiores, a maioria delas pode ser conduzida com sucesso. 
É importante ressaltar que significância estatística de um efeito nem sempre sugere relevância clínica, ou igualmente, um resultado não significativo pode não representar a ineficácia de um tratamento. Todas as informações devem ser consideradas no contexto de variabilidade de pacientes e apresentações.

Atualmente, cirurgias desnecessárias parecem estar sendo realizadas em alguns pacientes idosos com CGP, o que pode implicar em aumento das taxas de morbimortalidade. Estudos recentes sugerem benefício, favorecendo prognóstico, em pacientes selecionados para tratamento endoscópico, considerando a idade e condições clínicas desfavoráveis. Apesar de não abordado nesta revisão, as modalidades de tratamento endoscópico demonstram melhores resultados perioperatórios com relação a tempo de procedimento, de internação e custos (Choi KS et al., 2011; Chiu et al., 2012; Park et al., 2014).

Assim, a endoscopia terapêutica desenvolveu-se, aperfeiçoou-se e teve sua aplicabilidade aumentada nos últimos anos em decorrência de progresso tecnológico, ampliação dos critérios adotados, diagnóstico precoce, entusiasmo da comunidade médica e mudanças de paradigmas relacionados aos aspectos clínicos oncológicos.

Apesar da existência das diretrizes de tratamento, é igualmente, ou mais importante, que pacientes com o diagnóstico de CGP sejam cuidados em centros de referência, habituados a grande volume de procedimentos e sede de profissionais especializados nas diferentes áreas envolvidas, seja qual for a terapêutica. Pacientes e profissionais da área da saúde são, primariamente, os mais interessados em obter benefícios com aceitáveis 
níveis de adversidades. Desta forma, a generalização a instituições não especializadas requer discernimento e prudência, objetivando aprimorar os resultados.

\subsection{Limitações}

As análises relatadas combinam dados e informações entre os estudos selecionados, avaliando os efeitos das diferentes formas de abordagens de tratamento do CGP de um modo mais preciso que numa pesquisa única. A vantagem observada é que se verifica, desta maneira, um aumento do nível de evidência de $2 \mathrm{~b}$ (estudos de coorte retrospectivos isolados) para $2 \mathrm{a}$ (revisões sistemáticas de estudos $2 \mathrm{~b}$ ), de acordo com Oxford Centre for Evidence-Based Medicine (CEBM, 2009).

Entende-se por viés de publicação a tendência de os resultados publicados estarem sistematicamente diferentes da realidade. Ele refere-se a um fenômeno em comunicação científica baseado na propensão dos autores e editores a publicar estudos com resultados positivos (ou seja, os resultados que mostram um achado significativo) em detrimento dos com achados negativos (ou seja, apoiando a hipótese nula) ou dos que não suportam os resultados esperados (Dickersin, 1990; Song et al., 2010). Como resultado, achados importantes, ainda que negativos, podem não atingir um impacto adequado (McGauran et al., 2010).

Os riscos de vieses foram detectados na análise dos funnel plots para os desfechos de sobrevida de cinco e 10 anos, complicações, ressecção completa e recorrência. 
Uma das limitações desta metanálise é a presença de heterogeneidade relacionada a estes desfechos, possivelmente referente a um pequeno número de estudos disponíveis. Mesmo buscando-se e incluindo-se pesquisas metodologicamente semelhantes, resultados positivos ou negativos (estatisticamente significativos ou não) por influência do acaso, ainda podem ocorrer.

Apesar da realização da análise de sensibilidade, algum grau de heterogeneidade em metanálise médica é inevitável, podendo seu impacto indeterminar a qualidade e legitimidade dos resultados obtidos. Uma simples categorização de valores para $I^{2}$ não seria adequada para quaisquer circunstâncias, apesar de adjetivos como baixo, moderado e alto, para índices de 25\%, 50\%, e 75\%, serem habitualmente empregados (Higgins et al., 2003). A quantificação de heterogeneidade é apenas um componente de uma gama de possibilidades relativas às variações e diferenças entre estudos. Como descrito previamente, o grau de inconsistência pode determinar implicações clínicas.

O caráter retrospectivo dos estudos avaliados é também uma limitação, comparado às informações obtidas de bancos de dados prospectivos e randomizados. Neste trabalho, a escolha entre a ressecção endoscópica ou cirurgia não se baseou em randomização. Todos os estudos incluídos foram desenvolvidos na Ásia, região em que a prevalência de CGP é elevada e os centros de tratamento altamente desenvolvidos, sede de profissionais treinados e tecnologia avançada. Assim, pesquisas semelhantes, reproduzidas em países ocidentais, são de grande valia. 
A análise conjunta dos pacientes submetidos à ressecção endoscópica, considerando-se os critérios de indicação absolutos e expandidos, pode representar uma limitação. Uma vez que somente um trabalho (Kim et al., 2015) aborda a comparação isolada dos indivíduos que preenchem os critérios expandidos com os submetidos à cirurgia, conclusões a esse respeito podem ser precipitadas.

Alguns trabalhos utilizaram-se do pareamento por escore de propensão (do inglês, propensity-score matching) com o objetivo de minimizar vieses de seleção como, por exemplo, o descrito por Park et al. (2014). Nestas situações, são aplicados métodos de análise que permitem gerenciar o potencial confundimento na relação entre os fatores e o desfecho. O pareamento de pacientes entre os grupos faz com que as distribuições sejam semelhantes, tornando os grupos comparáveis, de modo que as associações estimadas possam ser atribuídas apenas àquela abordagem.

Mesmo seguindo-se sistematização metodológica adequada, de modo a reduzir os riscos de vieses, outros erros estatísticos ainda podem permanecer sob influência de fatores de confusão não mensuráveis (do inglês, confounding factor), determinando influência variável nas informações obtidas. Alguns dados importantes podem não estar explícitos ou bem definidos nos artigos, até mesmo inexistindo, dificultando a extração acurada e fidedigna de resultados, assim como conclusões adequadas e confiáveis. 
6 Conclusões 
Estabelecendo comparações e analisando os dados disponíveis sobre os desfechos de curto e longo prazos da abordagem endoscópica comparada à cirúrgica no tratamento dos pacientes com CGP, esta revisão conclui que:

- As taxas de sobrevida de três, cinco e 10 anos, recorrência e mortalidade são semelhantes.

- As taxas de complicações observadas são menores nos pacientes submetidos à abordagem endoscópica.

- As taxas de ressecção completa são maiores nos pacientes pertencentes ao grupo de tratamento cirúrgico.

- Em razão das conclusões acima e, havendo infraestrutura adequada, pode-se propor a ressecção endoscópica como forma de tratamento inicial de pacientes selecionados com o diagnóstico de CGP. 
7 Anexos 


\section{Anexo A - Sign Methodology Checklist 3: Cohort Studies ${ }^{5}$}

Study identification (include author, title, year of publication, journal title, pages)

Guideline topic:

Key question number:

Reviewer:

Before completing this checklist, consider:

1. Is this paper really a cohort study? If in doubt, check the study design algorithm available from SIGN and make sure you have the correct checklist.

2. Is the paper relevant to key question? Analyze using PICO (Patient or Population, Intervention, Comparison, Outcome). If no, reject (give reason below). If yes, complete the checklist.

Reason for rejection: 1. Paper not relevant to key question ( ) 2. Other reason ( ) (please specify)

PLEASE NOTE THAT A RETROSPECTIVE STUDY (I.E., DATABASE OR CHART STUDY) CANNOT BE RATED HIGHER THAN +.

\section{SECTION 1: INTERNAL VALIDITY}

In a well conducted cohort study. Does this study do it?

1.1. The study addresses an appropriate and clearly focused question.

Yes ( ) No ( ) Can't say ( )

\section{SELECTION OF SUBJECTS}

1.2. The two groups being studied are selected from source populations that are comparable in all respects other than the factor under investigation.

Yes ( ) No ( ) Can't say ( ) Does not apply ( )

1.3. The study indicates how many of the people asked to take part did so, in each of the groups being studied.

Yes ( ) No ( ) Does not apply ()

1.4. The likelihood that some eligible subjects might have the outcome at the time of enrolment is assessed and taken into account in the analysis.

Yes ( ) No ( ) Can't say ( ) Does not apply ( )

1.5. What percentage of individuals or clusters recruited into each arm of the study dropped out before the study was completed. $\%$

1.6. Comparison is made between full participants and those lost to follow up, by exposure status.

Yes ( ) No ( ) Can't say ( ) Does not apply ( )

\section{ASSESSMENT}

1.7. The outcomes are clearly defined.

Yes ( ) No ( ) Can't say ( )

1.8. The assessment of outcome is made blind to exposure status. If the study is retrospective this may not be applicable.

Yes ( ) No ( ) Can't say ( ) Does not apply ( )

5 Os anexos A, B, C e D foram mantidos em inglês de modo a garantir correspondência rigorosa entre os documentos originais e os reproduzidos. 
1.9. Where blinding was not possible, there is some recognition that knowledge of exposure status could have influenced the assessment of outcome.

Yes ( ) No ( ) Can't say ( )

1.10. The method of assessment of exposure is reliable.

$$
\text { Yes ( ) No ( ) Can't say () }
$$

1.11. Evidence from other sources is used to demonstrate that the method of outcome assessment is valid and reliable.

Yes ( ) No ( ) Can't say ( ) Does not apply ( )

1.12. Exposure level or prognostic factor is assessed more than once.

Yes ( ) No ( ) Can't say ( ) Does not apply ( )

\section{CONFOUNDING}

1.13. The main potential confounders are identified and taken into account in the design and analysis.

Yes ( ) No ( ) Can't say ( )

\section{STATISTICAL ANALYSIS}

1.14. Have confidence intervals been provided? Yes ( ) No ( )

\section{SECTION 2. OVERALL ASSESSMENT OF THE STUDY}

2.1 How well was the study done to minimize the risk of bias or confounding? High-quality (++) ( ) Acceptable (+) () Unacceptable (0) ()

2.2. Taking into account clinical considerations, your evaluation of the methodology used, and statistical power of the study, do you think there is clear evidence of an association between exposure and outcome?

Yes ( ) No ( ) Can't say ( )

2.3. Are the results of this study directly applicable to the patient group targeted in this guideline?

Yes ( ) No ( )

2.4. NOTES. Summarize the authors' conclusions. Add any comments on your own assessment of the study, and the extent to which it answers your questions and mention any areas of uncertainty raised above. 


\section{Anexo B - Script for Critical Assessment of Evidence}

\section{CRITICAL ANALYSIS OF SELECTED EVIDENCE}

REFERENCE 1 - STRENGTH OF EVIDENCE (describe each item)

1) Included and excluded population

2) Randomization and allocation method

3) Intervention, comparison and blinding

4) Primary outcome and follow-up period

5) Secondary outcomes

6) Sample calculation and randomized population

7) Analyzed data (intervention and comparison)

8) Losses, migration and prognostic differences

RESULTS

BENEFIT AND/OR HARM - ABSOLUTE DATA

\begin{tabular}{|c|c|c|c|c|c|c|c|}
\hline Outcomes & N/NEE & N/NES & ARE & ARS & ARR/ARI & NNT/NNH & $95 \% \mathrm{Cl}$ \\
\hline & & & & & & & \\
\hline & & & & & & & \\
\hline
\end{tabular}

BENEFIT AND/OR HARM - AVERAGE

\begin{tabular}{|l|l|l|l|l|}
\hline Outcomes & Intervention & Comparison & Relationship & Significance \\
\hline & & & & \\
\hline & & & & \\
\hline
\end{tabular}

$\mathrm{N}$ : number of patients analyzed; NEE: number of events in the endoscopic treatment; NES: number of events in the surgical treatment; ARE: absolute risk in endoscopy; ARS: absolute risk in surgery; ARR: absolute risk reduction; ARI: absolute risk increase; NNT: number needed to treat; $\mathrm{NNH}$ : number needed to harm; $95 \% \mathrm{Cl}$ : $95 \%$ confidence interval

\section{EVIDENCE SYNTHESIS}

IN PATIENTS, THE INTERVENTION COMPARED TO REDUCES (OR INCREASES) THE OUTCOME RISK. 


\section{Anexo C- Newcastle - Ottawa Quality Assessment Scale for Cohort Studies}

Note: A study can be awarded a maximum of one star for each numbered item within the Selection and Outcome categories. A maximum of two stars can be given for Comparability.

\section{SELECTION}

1) Representativeness of the exposed cohort
a) truly representative of the average in the community. *
b) somewhat representative of the average in the community. *
c) selected group of users eg nurses, volunteers.
d) no description of the derivation of the cohort.

2) Selection of the non exposed cohort
a) drawn from the same community as the exposed cohort. *
b) drawn from a different source.
c) no description of the derivation of the non exposed cohort.

3) Ascertainment of exposure
a) secure record (eg surgical records). *
b) structured interview. *
c) written self report.
d) no description.

4) Demonstration that outcome of interest was not present at start of study
a) yes. *
b) no.

\section{COMPARABILITY}

1) Comparability of cohorts on the basis of the design or analysis
a) study controls for (select the most important factor). *
b) study controls for any additional factor. * (This criteria could be modified to indicate specific control for a second important factor.)

\section{OUTCOME}

1) Assessment of outcome
a) independent blind assessment. *
b) record linkage. *
c) self report.
d) no description.

2) Was follow-up long enough for outcomes to occur?
a) yes (select an adequate follow up period for outcome of interest). *
b) no.

3) Adequacy of follow up of cohorts
a) complete follow up - all subjects accounted for.
b) subjects lost to follow up unlikely to introduce bias - small number lost - > $\%$ (select an adequate \%) follow up, or description provided of those lost). *
c) follow up rate < $\%$ (select an adequate \%) and no description of those lost.
d) no statement. 


\section{Anexo D - Oxford Centre for Evidence-Based Medicine 2009 Levels of Evidences}

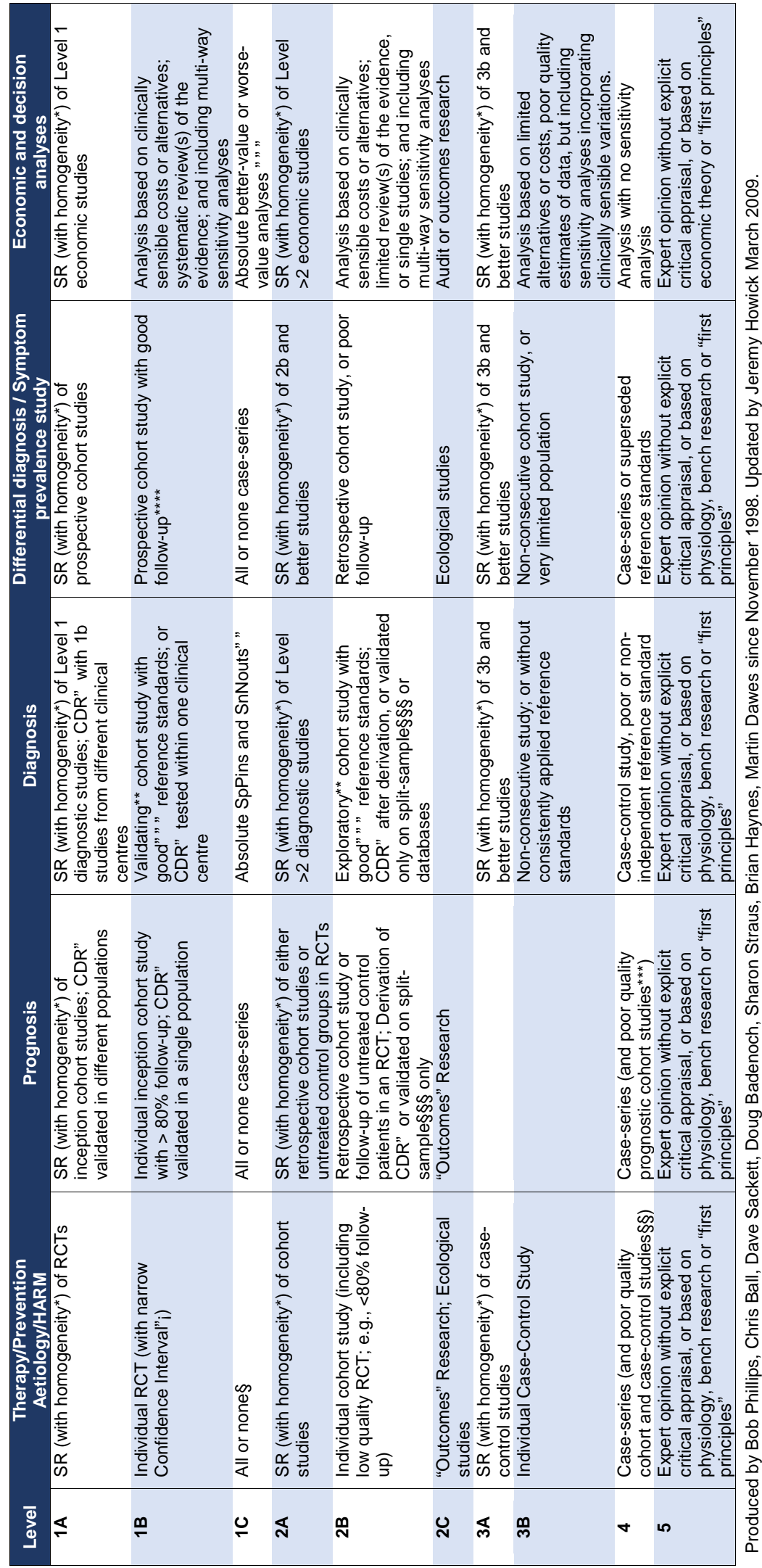




\section{NOTES:}

Users can add a minus-sign "-" to denote the level of that fails to provide a conclusive answer because:

- EITHER a single result with a wide Confidence Interval

- OR a Systematic Review with troublesome heterogeneity.

Such evidence is inconclusive, and therefore can only generate Grade D recommendations.

* By homogeneity we mean a systematic review that is free of worrisome variations (heterogeneity) in the directions and degrees of results between individual studies. Not all systematic reviews with statistically significant heterogeneity need be worrisome, and not all worrisome heterogeneity need be statistically significant. As noted above, studies displaying worrisome heterogeneity should be tagged with a "-" at the end of their designated level.

" Clinical Decision Rule. (These are algorithms or scoring systems that lead to a prognostic estimation or a diagnostic category.)

" $i \quad$ See note above for advice on how to understand, rate and use trials or other studies with wide confidence intervals.

$\S \quad$ Met when all patients died before the Rx became available, but some now survive on it; or when some patients died before the Rx became available, but none now die on it.

§§ By poor quality cohort study we mean one that failed to clearly define comparison groups and/or failed to measure exposures and outcomes in the same (preferably blinded), objective way in both exposed and non-exposed individuals and/or failed to identify or appropriately control known confounders and/or failed to carry out a sufficiently long and complete follow-up of patients. By poor quality case-control study we mean one that failed to clearly define comparison groups and/or failed to measure exposures and outcomes in the same (preferably blinded), objective way in both cases and controls and/or failed to identify or appropriately control known confounders.

§§§ Split-sample validation is achieved by collecting all the information in a single tranche, then artificially dividing this into "derivation" and "validation" samples.

" " An "Absolute SpPin" is a diagnostic finding whose Specificity is so high that a Positive result rules-in the diagnosis. An "Absolute SnNout" is a diagnostic finding whose Sensitivity is so high that a Negative result rules-out the diagnosis.

"i" $\mathrm{i}$ Good, better, bad and worse refer to the comparisons between treatments in terms of their clinical risks and benefits.

" " " Good reference standards are independent of the test, and applied blindly or objectively to applied to all patients. Poor reference standards are haphazardly applied, but still independent of the test. Use of a non-independent reference standard (where the 'test' is included in the 'reference', or where the 'testing' affects the 'reference') implies a level 4 study.

" " " " Better-value treatments are clearly as good but cheaper, or better at the same or reduced cost. Worsevalue treatments are as good and more expensive, or worse and the equally or more expensive.

** Validating studies test the quality of a specific diagnostic test, based on prior evidence. An exploratory study collects information and trawls the data (e.g. using a regression analysis) to find which factors are 'significant'.

*** By poor quality prognostic cohort study we mean one in which sampling was biased in favour of patients who already had the target outcome, or the measurement of outcomes was accomplished in $<80 \%$ of study patients, or outcomes were determined in an unblinded, non-objective way, or there was no correction for confounding factors.

**** Good follow-up in a differential diagnosis study is $>80 \%$, with adequate time for alternative diagnoses to emerge (for example 1-6 months acute, 1 - 5 years chronic) 


\section{GRADES OF RECOMMENDATION}
A consistent level 1 studies
B consistent level 2 or 3 studies or extrapolations from level 1 studies
C level 4 studies or extrapolations from level 2 or 3 studies
D level 5 evidence or troublingly inconsistent or inconclusive studies of any level

"Extrapolations" are where data is used in a situation that has potentially clinically important differences than the original study situation. 


\section{Anexo E - Ata de Aprovação do Projeto}

\section{Prifilatin \\ MEDICINA \\ TSP \\ COMITÊ DE ÉTICA EM PESQUISA}

\section{APROVAÇÃo}

O Comitê de Ética em Pesquisa da Faculdade de Medicina da Universidade de São Paulo, em o6.o2.15 tomou ciência dos documentos apresentados ao Projeto $\mathrm{n}^{\circ} \mathbf{0 3 7 / 1 5}$ intitulado ENDOSCOPIA E CIRURGIA NO TRATAMENTO DO CÂNCER GÁSTRICO PRECOCE (CGP): REVISÃo SISTEMÁTICA E METANÁLISE.”, apresentado pelo Departamento de Gastroenterologia , e por não haver nenhum óbice ético , a proposta foi aprovada.

Pesquisador(a) Responsável: Eduardo Guimaraes H. de Moura

Pesquisador(a) Executante : André Kondo

CEP-FMUSP, o6 de fevereiro 2015.

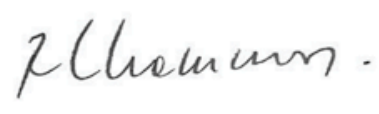

Prof. Dr.Roger Chammas

$$
\text { Coordenador }
$$

Comitê de Ética em Pesquisa 


\title{
Anexo F - Termo de Cessão de Direitos Autorais das llustrações
}

\author{
TERMO DE CESSÃO DE DIREITOS AUTORAIS DAS ILUSTRAÇÕES
}

Pelo presente instrumento, eu, GUSTAVO LUIS RODELA SILVA, na qualidade de titular dos direitos de autor das ilustrações utilizadas nesta tese, autorizo o pós-graduando ANDRÉ KONDO a inclui-las na sua publicação.

Declaro estar ciente de que o material acima identificado será objeto de divulgação em meio acadêmico na Faculdade de Medicina da Universidade de São Paulo.

Declaro, ainda, expressamente, que as ilustrações de que trata este termo de autorização são de minha autoria, não violando direitos de terceiros.

Por ser a expressão da verdade, dato e assino o presente termo de autorização.

São Paulo, 26 de julho de 2016.

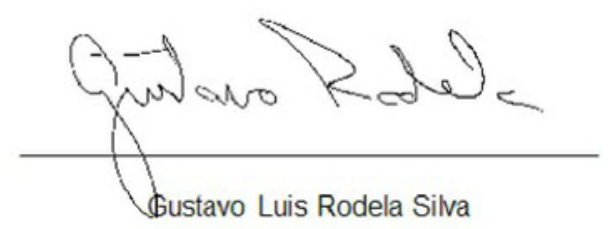




\section{REFERÊNCIAS}


Abe S, Oda I, Suzuki H, Nonaka S, Yoshinaga S, Odagaki T, Taniguchi H, Kushima R, Saito Y. Short- and long-term outcomes of endoscopic submucosal dissection for undifferentiated early gastric cancer. Endoscopy. 2013; 45(9):703-7.

Ahn JY, Jung HY, Choi KD, Kim MY, Lee JH, Choi KS, Kim DH, Song HJ, Lee $\mathrm{GH}$, Kim JH, Park YS. Endoscopic and oncologic outcomes after endoscopic resection for early gastric cancer: 1370 cases of absolute and extended indications. Gastrointest Endosc. 2011; 74(3):485-93.

Alves JR, Lopes LR, Loureiro MP, Andreollo NA. Laparoscopy-assisted partial gastrectomy for early gastric cancer. Arq Bras Cir Dig. 2011; 24(3):235-8.

An JY, Baik YH, Choi MG, Noh JH, Sohn TS, Kim S. Predictive factors for lymph node metastasis in early gastric cancer with submucosal invasion: analysis of a single institutional experience. Ann Surg. 2007; 246(5):749-53.

Anderson WF, Camargo MC, Fraumeni JF Jr, Correa P, Rosenberg PS, Rabkin CS. Age-specific trends in incidence of noncardia gastric cancer in US adults. JAMA. 2010; 303(17):1723-8. 
Andreollo NA, Lopes LR, Coelho-Neto JS. Postoperative complications after total gastrectomy in the gastric cancer. Analysis of 300 patients. Arq Bras Cir Dig. 2011; 24(2):126-30.

ASGE Technology Committee. Maple JT, Abu Dayyeh BK, Chauhan SS, Hwang JH, Komanduri S, Manfredi M, Konda V, Murad FM, Siddiqui UD, Banerjee S. Endoscopic submucosal dissection. Gastrointest Endosc. 2015; 81(6):1311-25.

Ballantyne KC, Morris DL, Jones JA, Gregson RH, Hardcastle JD. Accuracy of identification of early gastric cancer. Br J Surg. 1987; 74(7):618-9.

Barchi LC, Jacob CE, Bresciani CJC, Yagi OK, Mucerino DR, Lopasso FP, Mester M, Ribeiro-Júnior U, Dias AR, Ramos MF, Cecconello I, Zilberstein B. Minimally invasive surgery for gastric cancer: time to change the paradigm. Arq Bras Cir Dig. 2016; 29(2):117-20.

Bresciani CJC, Gama-Rodrigues JJ, Jacob CE, Perez RO, Bresciani EH, Bresciani $\mathrm{BH}$, Ribeiro-Júnior $\mathrm{U}$, Zilberstein $\mathrm{B}$. Indicações e técnicas do tratamento laparoscópico do câncer gástrico. Rev Bras Videocir. 2004; 2(4):206-13.

Bresciani CJC, Perez RO, Jacob CE, Gama-Rodrigues J, Zilberstein B, Cecconello I. Endogastric surgery for gastric diseases - simplifying technical aspects. Surg Laparosc Endosc Percutan Tech. 2007; 17(5):407-12.

Cao Y, Liao C, Tan A, Gao Y, Mo Z, Gao F. Meta-analysis of endoscopic submucosal dissection versus endoscopic mucosal resection for tumors of the gastrointestinal tract. Endoscopy. 2009; 41(9):751-7. 
Caruso S, Patriti A, Roviello F, De Franco L, Franceschini F, Coratti A, Ceccarelli G. Laparoscopic and robot-assisted gastrectomy for gastric cancer: Current considerations. World J Gastroenterol. 2016; 22(25):5694717.

CEBI - Centre for Evidence Based Intervention, University of Oxford. How to read a forest plot. Disponível em: <http://www.cebi.ox.ac.uk/forpractitioners/what-is-good-evidence/how-to-read-a-forest-plot.html>. Acesso em: 18 mai. 2015.

CEBM - Centre for Evidence-Based Medicine. Levels of evidence, 2009. Disponível em: <http://www.cebm.net/oxfordcentre-evidence-basedmedicine-levels-evidence-march-2009/>. Acesso em: 18 mai. 2015.

Centre for Evidence-Based Medicine, Headington, Oxford. Critically Appraised Topics (CAT). Disponível em: <http://www.cebm.net/catmakerebm-calculators/>. Acesso em: 25 jul. 2015.

Chang KJ, Katz KD, Durbin TE, Erickson RA, Butler JA, Lin F, Wuerker RB. Endoscopic ultrasound-guided fine-needle aspiration. Gastrointest Endosc. 1994; 40(6):694-9.

Chaves DM, Maluf Filho F, de Moura EG, Santos ME, Arrais LR, Kawaguti F, Sakai P. Endoscopic submucosal dissection for the treatment of early esophageal and gastric cancer - initial experience of a western center. Clinics (Sao Paulo). 2010; 65(4):377-82. 
Chaves DM, Moura EG, Milhomem D, Arantes VN, Yamazaki K, Maluf F, Albuquerque W, Conrado AC, Araújo JC, Uejo PH, Sakai P. Initial experience of endoscopic submucosal dissection in Brazil to treat early gastric and esophagheal cancer: a multi-institutional analysis. Arq Gastroenterol. 2013; 50(2):148-52.

Chiu PW, Teoh AY, To KF, Wong SK, Liu SY, Lam CC, Yung MY, Chan FK, Lau JY, Ng EK. Endoscopic submucosal dissection (ESD) compared with gastrectomy for treatment of early gastric neoplasia: a retrospective cohort study. Surg Endosc. 2012; 26(12): 3584-91.

Chiu PW. Novel endoscopic therapeutics for early gastric cancer. Clin Gastroenterol Hepatol. 2014; 12(1):120-5.

Cho KB, Jeon WJ, Kim JJ. Worldwide experiences of endoscopic submucosal dissection: not just Eastern acrobatics. World J Gastroenterol. $2011 ; 17(21): 2611-7$

Choi IJ, Lee JH, Kim YI, Kim CG, Cho SJ, Lee JY, Ryu KW, Nam BH, Kook MC, Kim YW. Long-term outcome comparison of endoscopic resection and surgery in early gastric cancer meeting the absolute indication for endoscopic resection. Gastrointest Endosc. 2015; 81(2):333-41.e1.

Choi J, Kim SG, Im JP, Kim JS, Jung HC, Song IS. Endoscopic prediction of tumor invasion depth in early gastric cancer. Gastrointest Endosc. 2011; 73(5):917-24. 
Choi KS, Jung HY, Choi KD, Lee GH, Song HJ, Kim DH, Lee JH, Kim MY, Kim BS, Oh ST, Yook JH, Jang SJ, Yun SC, Kim SO, Kim JH. EMR versus gastrectomy for intramucosal gastric cancer: comparison of long-term outcomes. Gastrointest Endosc. 2011; 73(5): 942-8.

Chung MW, Jeong O, Park YK, Lee KH, Lee JH, Lee WS, Joo YE, Choi SK, Cho SB. Comparison on the long term outcome between endoscopic submucosal dissection and surgical treatment for undifferentiated early gastric cancer. Korean J Gastroenterol. 2014; 63(2):90-8.

Correa P, Piazuelo MB, Wilson KT. Pathology of gastric intestinal metaplasia: clinical implications. Am J Gastroenterol. 2010; 105(3):493-8.

Coutinho ESF, Cunha GM. Basic concepts in epidemiology and statistics for reading controlled clinical trials. Rev Bras Psiquiatr. 2005; 27(2):146-51.

de Vries AC, Haringsma J, de Vries RA, Ter Bog F, van Grieken NC, Meijer GA, van Dekken H, Kuipers EJ. Biopsy strategies for endoscopic surveillance of pre-malignant gastric lesions. Helicobacter. 2010; 15(4):259-64.

Dickersin K. The existence of publication bias and risk factors for its occurrence. JAMA. 1990; 263(10):1385-9. 
Dinis-Ribeiro M, Areia M, de Vries AC, Marcos-Pinto R, Monteiro-Soares M, O'Connor A, Pereira C, Pimentel-Nunes P, Correia R, Ensari A, Dumonceau JM, Machado JC, Macedo G, Malfertheiner P, Matysiak-Budnik T, Megraud F, Miki K, O'Morain C, Peek RM, Ponchon T, Ristimaki A, Rembacken B, Carneiro F, Kuipers EJ; European Society of Gastrointestinal Endoscopy; European Helicobacter Study Group; European Society of Pathology; Sociedade Portuguesa de Endoscopia Digestiva. Management of precancerous conditions and lesions in the stomach (MAPS): guideline from the European Society of Gastrointestinal Endoscopy (ESGE), European Helicobacter Study Group (EHSG), European Society of Pathology (ESP), and the Sociedade Portuguesa de Endoscopia Digestiva (SPED). Endoscopy. 2012; 44(1):74-94.

Eckardt VF, Giessler W, Kanzler G, Remmele W, Bernhad G. Clinical and morphological characteristics of early gastric cancer. A case-control study. Gastroenterology. 1990; 98(3):708-14.

Edge SB, Byrd DR, Compton CC, et al (Eds). American Joint Committee on Cancer Staging Manual. $7^{\text {th }}$ Ed. New York: Springer-Verlag, 2009. p.117-26.

Ell C, Gossner L, May A, Schneider HT, Hahn EG, Stolte M, Sroka R. Photodynamic ablation of early cancers of the stomach by means of mTHPC and laser irradiation: preliminary clinical experience. Gut. 1998; 43(3):345-9.

El-Sedfy A, Brar SS, Coburn NG. Current role of minimally invasive approaches in the treatment of early gastric cancer. World J Gastroent. 2014; 20(14): 3880-8. 
Etoh T, Katai H, Fukagawa T, Sano T, Oda I, Gotoda T, Yoshimura K, Sasako M. Treatment of early gastric cancer in the elderly patient: results of EMR and gastrectomy at a national referral center in Japan. Gastrointest Endosc. 2005; 62(6): 868-71.

Everett SM, Axon AT. Early gastric cancer in Europe. Gut. 1997; 41(2):142-50.

Everett SM, Axon AT. Early gastric cancer: disease or pseudo-disease? Lancet. 1998; 351(9112):1350-2.

Ezoe Y, Muto M, Uedo N, Doyama H, Yao K, Oda I, Kaneko K, Kawahara Y, Yokoi C, Sugiura Y, Ishikawa H, Takeuchi Y, Kaneko Y, Saito Y. Magnifying narrow-band imaging is more accurate than conventional white-light imaging in diagnosis of gastric mucosal cancer. Gastroenterology. 2011; 141(6):201725.e3.

Facciorusso A, Antonino M, Di Maso M, Muscatiello N. Endoscopic submucosal dissection vs endoscopic mucosal resection for early gastric cancer: a meta-analysis. World J Gastrointest Endosc. 2014; 6(11):555-63.

Fenoglio-Preiser C, Carneiro F, Correa P, et al. Tumours of the stomach. In: Hamilton R, Aalthonen L (eds.). Pathology and genetics of tumours of the digestive system. Lyon: International Agency for Research on Cancer, 2000. p. 48.

Ferlay J, Shin HR, Bray F, Forman D, Mathers C, Parkin DM. Estimates of worldwide burden of cancer in 2008: GLOBOCAN 2008. Int J Cancer. 2010; 127(12):2893-917. 
Folli S, Dente M, Dell'Amore D, Gaudio D, Nanni O, Saragoni L, Vio A. Early gastric cancer: prognostic factors in 223 patients. Br J Surg. 1995; 82(7):9526.

Folli S, Morgagni P, Roviello F, De Manzoni G, Marrelli D, Saragoni L, Di Leo A, Gaudio M, Nanni O, Carli A, Cordiano C, Dell'Amore D, Vio A. Risk factors for lymph node metastases and their prognostic significance in early gastric cancer (EGC) for the Italian Research Group for Gastric Cancer (IRGGC). Jpn J Clin Oncol. 2001; 31(10):495-9.

Fujikawa H, Sakamaki K, Kawabe T, Hayashi T, Aoyama T, Sato T, Oshima T, Rino Y, Morita S, Masuda M, Ogata T, Cho H, Yoshikawa T. A new statistical model identified two-thirds of clinical T1 gastric cancers as possible candidates for endoscopic treatment. Ann Surg Oncol. 2015; 22(7):2317-22.

Fukase K, Matsuda T, Suzuki M, Toda H, Okuyama Y, Sakai JI, Saito H, Sato SI, Mito S. Evaluation of the efficacy of endoscopic treatment for gastric cancer considered in terms of long-term prognosis: a comparison with surgical treatment. Dig Endosc. 1994; 6(3): 241-7.

Fukunaga S, Machida $\mathrm{H}$, Tominaga $\mathrm{K}$, et al. Short- and long-term prognosis of patients with early gastric cancer: comparative analysis between endoscopic submucosal dissection and surgical operation [Abstract]. Gastrointest Endosc. 2012; 75(Suppl.):234-5. 
Fukunaga S, Nagami Y, Shiba M, Ominami M, Tanigawa T, Yamagami H, Tanaka H, Muguruma K, Watanabe T, Tominaga K, Fujiwara Y, Ohira M, Hirakawa K, Arakawa T. Long-term prognosis of expanded-indication differentiated early gastric cancer treated with endoscopic submucosal dissection or surgery using propensity score analysis. Gastrointest Endosc. 2016; pii: S0016-5107(16)30333-9.

Gama-Rodrigues JJ, Bresciani CJC, Waitzberg DL, Matsuda M, Iriya K, Pinotti HW. Surgical management of gastric carcinoma: extent of gastric resection and lymphadenectomy - current trends and results. Arq Bras Cir Dig .1986; 1(3):84-9.

Gama-Rodrigues JJ, Bresciani CJC, Waitzberg DL, Matsuda M, Malzoni CE, Pinotti HW. Resultados do tratamento do câncer gástrico precoce. Arq Bras Cir Dig. 1992; 7:86-9.

Gotoda T, Yamamoto H, Soetikno RM. Endoscopic submucosal dissection of early gastric cancer. J Gastroenterol. 2006; 41(10):929-42.

Gotoda T, Yanagisawa A, Sasako M, Ono H, Nakanishi Y, Shimoda T, Kato Y. Incidence of lymph node metastasis from early gastric cancer: estimation with a large number of cases at two large centers. Gastric Cancer. 2000; 3(4):219-25.

Gotoda T. Endoscopic resection of early gastric cancer. Gastric Cancer. 2007; 10(1): 1-11.

Gotoda T. Endoscopic resection of early gastric cancer: the Japanese perspective. Curr Opin Gastroenterol. 2006; 22(5):561-9. 
Gunaratnam NT, Kathawala M, Gostout C, Saltzman JR, Travis AC. Overview of endoscopic resection of gastrointestinal tumors. Up-to-date. Disponível em: <http://www.uptodate.com/contents/overview-of-endoscopicresection-of-gastrointestinal-tumors?source=see_link\&sectionName=Gastric +cancer\&anchor=H3\#H3>. Acesso em: 10 dez. 2015.

Higashimaya M, Oka S, Tanaka S, Sanomura Y, Yoshida S, Hiyama T, Arihiro K, Shimamoto F, Chayama K. Outcome of endoscopic submucosal dissection for gastric neoplasm in relationship to endoscopic classification of submucosal fibrosis. Gastric Cancer. 2013; 16(3):404-10.

Higgins JP, Thompson SG, Deeks JJ, Altman DG. Measuring inconsistency in meta-analyses. BMJ. 2003; 327(7414):557-60.

Higgins JPT, Green S. Cochrane Handbook for Systematic Reviews of Interventions. The Cochrane collaboration. Version 5.1.0, 2011. Disponível em: <http://handbook.cochrane.org/front_page.htm>. Acesso em: 15 jan. 2016.

Hiki Y, Shimao H, Mieno H, Sakakibara Y. Laser therapy for early upper gastrointestinal carcinoma. Surg Clin North Am. 1992; 72(3):571-80.

Hirasaki S, Tanimizu M, Nasu J, Shinji T, Koide N. Treatment of elderly patients with early gastric cancer by endoscopic submucosal dissection using an insulated-tip diathermic knife. Intern Med. 2005; 44(10):1033-8.

IACR - International Association of Cancer Registry: GLOBOCAN, 2012. Disponível em: <http://www.iacr.com.fr/index.php?option=com_content\&view =article\&id=101\&ltemid=578>. Acesso em: 18 mai. 2014. 
INCA - Instituto Nacional de Câncer José Alencar Gomes da Silva. Disponível em: <http://www2.inca.gov.br/wps/wcm/connect/tiposdecancer/ site/home/ estomago/definicao>. Acesso em: 12 nov. 2015.

Isomoto $\mathrm{H}$, Ohnita K, Yamaguchi N, Fukuda E, Ikeda K, Nishiyama $\mathrm{H}$, Akiyama M, Ozawa E, Nakao K, Kohno S, Shikuwa S. Clinical outcomes of endoscopic submucosal dissection in elderly patients with early gastric cancer. Eur J Gastroenterol Hepatol. 2010; 22(3):311-7.

Jacob CE, Bresciani CJC, Gama-Rodrigues JJ, Yagi OK, Mucerino DR, Zilberstein B, Cecconello I. Behavior of gastric cancer in brazilian population. Arq Bras Cir Dig. 2009; 22(1):29-32.

Jacob CE, Gama-Rodrigues JJ, Iriya K, Bresciani CJC, Zilberstein B, Martins BC. Early onset gastric cancer: complications and mortality rates subsequent to gastrectomy and lymphadenectomy: a single institution experience on 178 cases. Arq Bras Cir Dig. 2006; 19(4):146-52.

Jacob CE. Câncer gástrico precoce: estudo dos parâmetros clínicos e histopatológicos; correlação com a sobrevivência a longo prazo [Tese]. São Paulo: Faculdade de Medicina, Universidade de São Paulo, 2003.

JGCA - Japanese Gastric Cancer Association. Japanese Classification of Gastric Carcinoma. $2^{\text {nd }}$ English Edition. Gastric Cancer. 1998; 1(1):10-24.

JGCA - Japanese Gastric Cancer Association. Japanese Classification of Gastric Carcinoma. $3^{\text {rd }}$ English Edition. Gastric Cancer. 2011a; 14(2):101-2. 
JGCA - Japanese Gastric Cancer Association. Japanese gastric cancer treatment guidelines 2010 (ver. 3). Gastric Cancer. 2011b; 14(2):113-23.

Kajitani T. The general rules for the gastric cancer study in surgery and pathology. Part I. Clinical classification. Jpn J Surg. 1981; 11(2):127-39.

Kakushima N, Fujishiro M, Kodashima S, Muraki Y, Tateishi A, Yahagi N, Omata M. Technical feasibility of endoscopic submucosal dissection for gastric neoplasms in the elderly Japanese population. J Gastroenterol Hepatol. 2007; 22(3): 311-4.

Kang HJ, Kim DH, Jeon TY, Lee SH, Shin N, Chae SH, Kim GH, Song GA, Kim DH, Srivastava A, Park DY, Lauwers GY. Lymph node metastasis from intestinal-type early gastric cancer: experience in a single institution and reassessment of the extended criteria for endoscopic submucosal dissection. Gastrointest Endosc. 2010; 72(3):508-15.

Kato M, Kaise M, Yonezawa J, Toyoizumi H, Yoshimura N, Yoshida Y, Kawamura M, Tajiri H. Magnifying endoscopy with narrow-band imaging achieves superior accuracy in the differential diagnosis of superficial gastric lesions identified with white-light endoscopy: a prospective study. Gastrointest Endosc. 2010; 72(3):523-9.

Kim DY, Hong SJ, Cho GS, Jeong GA, Kim HK, Han JP, Lee YN, Ko BM, Lee MS. Long-term efficacy of endoscopic submucosal dissection compared with surgery for early gastric cancer: a retrospective cohort study. Gut Liver. $2014 ; 8(5): 519-25$. 
Kim HS, Lee DK, Baik SK, Kim JM, Kwon SO, Kim DS, Cho MY. Endoscopic mucosal resection with a ligation device for early gastric cancer and precancerous lesions: comparison of its therapeutic efficacy with surgical resection. Yonsei Med J. 2000; 41(5):577-83.

Kim JH, Song KS, Youn YH, Lee YC, Cheon JH, Song SY, Chung JB. Clinicopathologic factors influence accurate endosonographic assessment for early gastric cancer. Gastrointest Endosc. 2007; 66(5):901-8.

Kim YI, Kim YW, Choi IJ, Kim CG, Lee JY, Cho SJ, Eom BW, Yoon HM, Ryu $\mathrm{KW}$, Kook MC. Long-term survival after endoscopic resection versus surgery in early gastric cancers. Endoscopy. 2015; 47(4):293-301.

Kitamura T, Tanabe S, Koizumi W, Mitomi H, Saigenji K. Argon plasma coagulation for early gastric cancer: technique and outcome. Gastrointest Endosc. 2006; 63(1):48-54.

Kitano S, Shiraishi N, Uyama I, Sugihara K, Tanigawa N. Japanese Laparoscopic Surgery Study Group. A multicenter study on oncologic outcome of laparoscopic gastrectomy for early cancer in Japan. Ann Surg. 2007; 245:(1)68-72.

Kosaka T, Endo M, Toya Y, Abiko Y, Kudara N, Inomata M, Chiba T, Takikawa Y, Suzuki K, Sugai T. Long-term outcomes of endoscopic submucosal dissection for early gastric cancer: a single-center retrospective study. Dig Endosc. 2014; 26(2):183-91. 
Lauren P. The two histological main types of gastric carcinoma: diffuse and so-called intestinal-type carcinoma. An attempt at a histo-clinical classification. Acta Pathol Microbiol Scand. 1965; 64:31-49.

Lauren PA, Nevalainen TJ. Epidemiology of intestinal and diffuse types of gastric carcinoma. A time-trend study in Finland with comparison between studies from high- and low-risk areas. Cancer. 1993; 71(10):2926-33.

Lee CK, Chung IK, Lee SH, Kim SP, Lee SH, Lee TH, Kim HS, Park SH, Kim SJ, Lee $\mathrm{JH}$, Cho HD, Oh $\mathrm{MH}$. Is endoscopic forceps biopsy enough for a definitive diagnosis of gastric epithelial neoplasia? J Gastroenterol Hepatol. 2010; 25(9):1507-13.

Lee $\mathrm{HH}$, Yoo HM, Song KY, Jeon HM, Park CH. Risk of limited lymph node dissection in patients with clinically early gastric cancer: indications of extended lymph node dissection for early gastric cancer. Ann Surg Oncol. 2013; 20(11):3534-40.

Lee HJ, Yang HK, Ahn YO. Gastric cancer in Korea. Gastric Cancer. 2002; 5(3):177-82.

Lee $\mathrm{HL}$, Choi $\mathrm{CH}$, Cheung DY. Do we have enough evidence for expanding the indications of ESD for EGC? World J Gastroenterol. 2011; 17(21):2597601.

Lee IS, Yook JH, Park YS, Kim KC, Oh ST, Kim BS. Suitability of endoscopic submucosal dissection for treatment of submucosal gastric cancers. $\mathrm{Br} \mathrm{J}$ Surg. 2013; 100(5):668-73. 
Lee JH, Nam BH, Ryu KW, Ryu SY, Park YK, Kim S, Kim YW. Comparison of outcomes after laparoscopy-assisted and open total gastrectomy for early gastric cancer. Br J Surg. 2015; 102(12):1500-5.

Lee $\mathrm{JH}$, Yom CK, Han HS. Comparison of long-term outcomes of laparoscopy-assisted and open distal gastrectomy for early gastric cancer. Surg Endosc. 2009; 23(8):1759-63.

Lee JY, Choi IJ, Kim CG, Cho SJ, Kook MC, Ryu KW, Kim YW. Therapeutic Decision-Making Using Endoscopic Ultrasonography in Endoscopic Treatment of Early Gastric Cancer. Gut Liver. 2016; 10(1):42-50.

Li HY, Dai J, Xue HB, Zhao YJ, Chen XY, Gao YJ, Song Y, Ge ZZ, Li XB. Application of magnifying endoscopy with narrow-band imaging in diagnosing gastric lesions: a prospective study. Gastrointest Endosc. 2012; 76(6):112432.

Lian J, Chen S, Zhang Y, Qiu F. A meta-analysis of endoscopic submucosal dissection and EMR for early gastric cancer. Gastrointest Endosc. 2012; 76(4):763-70.

Liberati A, Altman DG, Tetzlaff J, Mulrow C, Gøtzsche PC, Ioannidis JP, Clarke M, Devereaux PJ, Kleijnen J, Moher D. The PRISMA statement for reporting systematic reviews and meta-analyses of studies that evaluate health care interventions: explanation and elaboration. $\mathrm{J}$ Clin Epidemiol. 2009; 62(10): e1-34. 
Lu W, Gao J, Yang J, Zhang Y, Lv W, Mu J, Dong P, Liu Y. Long-term clinical outcomes of laparoscopy-assisted distal gastrectomy versus open distal gastrectomy for early gastric cancer: A comprehensive systematic review and meta-analysis of randomized control trials. Medicine (Baltimore). 2016; 95(27): e3986.

Maehara Y, Orita H, Okuyama T, Moriguchi S, Tsujitani S, Korenaga D, Sugimachi K. Predictors of lymph node metastasis in early gastric cancer. $\mathrm{Br}$ J Surg. 1992; 79(3):245-7.

McGauran N, Wieseler B, Kreis J, Schüler Y, Kölsch H, Kaiser T. Reporting bias in medical research - a narrative review. Trials. 2010; 11:37.

Mimura S, Ito Y, Nagayo T, Ichii M, Kato H, Sakai H, Goto K, Noguchi Y, Tanimura H, Nagai Y, Suzuki S, Hiki Y, Hayata Y. Cooperative clinical trial of photodynamic therapy with photofrin II and excimer dye laser for early gastric cancer. Lasers Surg Med. 1996; 19(2):168-72.

Min YW, Min BH, Lee JH, Kim JJ. Endoscopic treatment for early gastric cancer. World J Gastroenterol. 2014; 20(16):4566-73.

Mochiki E, Kamiyama Y, Aihara R, Nakabayashi T, Asao T, Kuwano H. Laparoscopic assisted distal gastrectomy for early gastric cancer: Five years' experience. Surgery. 2005; 137(3):317-22.

Murakami T. Early cancer of the stomach. World J Surg. 1979; 3(6):685-92. 
Muto M, Katada C, Sano Y, Yoshida S. Narrow band imaging: a new diagnostic approach to visualize angiogenesis in superficial neoplasia. Clin Gastroenterol Hepatol. 2005; 3(7 Suppl. 1):S16-20.

Nakamura H, Yanai H, Nishikawa J, Okamoto T, Hirano A, Higaki M, Omori K, Yoshida T, Okita K. Experience with photodynamic therapy (endoscopic laser therapy) for the treatment of early gastric cancer. Hepatogastroenterology. 2001; 48(42):1599-603.

Nakamura K, Morisaki T, Sugitani A, Ogawa T, Uchiyama A, Kinukawa N, Tanaka M. An early gastric carcinoma treatment strategy based on analysis of lymph node metastasis. Cancer. 1999; 85(7):1500-5.

Nasu J, Nishina T, Hirasaki S, Moriwaki T, Hyodo I, Kurita A, Nishimura R. Predictive factors of lymph node metastasis in patients with undifferentiated early gastric cancers. J Clin Gastroenterol. 2006; 40(5):412-5.

NCCN - National Comprehensive Cancer Network Guidelines. Disponível em: <www.nccn.org/professionalsphysician_gls/f_guidelines.asp\#gastric>. Acesso em: 9 nov. 2015.

Nishida T, Haruma K, Tanaka S, Inoue K, Teshima H, Yoshihara M, Tari A, Sumii K, Kajiyama G. Comparison of endoscopic therapy and conventional surgery for the treatment of early gastric cancer in elderly patients. Nihon Ronen Igakkai Zasshi. 1993; 30(5):376-81. 
Noguchi Y, Yoshikawa T, Tsuburaya A, Motohashi H, Karpeh MS, Brennan MF. Is gastric carcinoma different between Japan and the United States? Cancer. 2000; 89(11):2237-46.

Oda I, Suzuki H, Nonaka S, Yoshinaga S. Complications of gastric endoscopic submucosal dissection. Dig Endosc. 2013; 25(Suppl 1):71-8.

Oguro Y. Laser endoscopic treatment for early gastric cancer. J Gastroenterol. 1994; 29(Suppl 7):91-5

Oka S, Tanaka S, Higashiyama M, Numata N, Sanomura Y, Yoshida S, Arihiro K, Chayama K. Clinical validity of the expanded criteria for endoscopic resection of undifferentiated-type early gastric cancer based on long-term outcomes. Surg Endosc. 2014; 28(2): 639-47.

Oka S, Tanaka S, Kaneko I, Mouri R, Hirata M, Kawamura T, Yoshihara M, Chayama K. Advantage of endoscopic submucosal dissection compared with EMR for early gastric cancer. Gastrointest Endosc. 2006; 64(6):877-83.

Ono H. Endoscopic submucosal dissection for early gastric cancer. Chin $\mathrm{J}$ Dig Dis. 2005; 6(3):119-21.

Ottawa Hospital Research Institute. The Newcastle-Ottawa Scale (NOS) for assessing the quality of nonrandomized studies in metaanalysis. Disponível em: <http://www.ohri.ca/programs/clinical_epidemiology/oxford.asp>. Acesso em: 20 jul. 2015. 
Park CH, Lee H, Kim DW, Chung H, Park JC, Shin SK, Hyung WJ, Lee SK, Lee YC, Noh SH. Clinical safety of endoscopic submucosal dissection compared with surgery in elderly patients with early gastric cancer: a propensity-matched analysis. Gastrointest Endosc. 2014; 80(4):599-609.

Park YM, Cho E, Kang HY, Kim JM. The effectiveness and safety of endoscopic submucosal dissection compared with endoscopic mucosal resection for early gastric cancer: a systematic review and metaanalysis. Surg Endosc. 2011; 25(8): 2666-77.

Parkin DM. The global health burden of infection associated cancers in the year 2002. Int J Cancer. 2006; 118(12):3030-44.

Peng LJ, Tian SN, Lu L, Chen H, Ouyang YY, Wu YJ. Outcome of endoscopic submucosal dissection for early gastric cancer of conventional and expanded indications: systematic review and meta-analysis. J Dig Dis. 2015;16(2):67-74.

Probst A, Pommer B, Golger D, Anthuber M, Arnholdt H, Messmann H. Endoscopic submucosal dissection in gastric neoplasia - experience from a European center. Endoscopy. 2010; 42(12):1037-44.

PROSPERO Centre for Reviews and Dissemination, University of York. "Guidance notes for registering a systematic review with PROSPERO". Disponível em: <http://www.crd.york.ac.uk/PROSPERO/>. Acesso em: 25 nov. 2014. 
Rodrigues CL, Ziegelmann PK. Meta-analysis: a practical guide. Rev HCPA. 2010; 30(4):436-47.

Roviello F, Rossi S, Marrelli D, Pedrazzani C, Corso G, Vindigni C, Morgagni P, Saragoni L, De Manzoni G, Tomezzoli A. Number of lymph node metastases and its prognostic significance in early gastric cancer: a multicenter Italian study. J Surg Oncol. 2006; 94(4):275-80.

Saito I, Tsuji Y, Sakaguchi Y, Niimi K, Ono S, Kodashima S, Yamamichi N, Fujishiro M, Koike K. Complications related to gastric endoscopic submucosal dissection and their managements. Clinical Endosc. 2014; 47(5):398-403.

Sakai P, Maluf Filho F, Iryia K, Moura EG, Tomishige T, Scabbia A, Ishioka S. An endoscopic technique for resection of small gastrointestinal carcinomas. Gastrointest Endosc. 1996; 44(1):65-8.

Sano T, Kobori O, Muto T. Lymph node metastasis from early gastric cancer: endoscopic resection of tumour. Br J Surg. 1992; 79(3):241-4.

Schlemper RJ, Riddell RH, Kato Y, Borchard F, Cooper HS, Dawsey SM, Dixon MF, Fenoglio-Preiser CM, Fléjou JF, Geboes K, Hattori T, Hirota T, Itabashi M, Iwafuchi M, Iwashita A, Kim YI, Kirchner T, Klimpfinger M, Koike M, Lauwers GY, Lewin KJ, Oberhuber G, Offner F, Price AB, Rubio CA, Shimizu M, Shimoda T, Sipponen P, Solcia E, Stolte M, Watanabe H, Yamabe H. The Vienna classification of gastrointestinal epithelial neoplasia. Gut. 2000; 47(2):251-5. 
Sedgwick P. How to read a forest plot in a meta-analysis. BMJ. 2015; 351:h4028.

Seto $\mathrm{Y}$, Nagawa H, Muto T. Impact of lymph node metastasis on survival with early gastric cancer. World J Surg. 1997; 21(2):186-9.

Shim CN, Song MK, Kang DR, Chung HS, Park JC, Lee H, Shin SK, Lee SK, Lee YC. Size discrepancy between endoscopic size and pathologic size is not negligible in endoscopic resection for early gastric cancer. Surg Endosc. $2014 ; 28(7): 2199-207$.

Shimamura Y, Ishii N, Nakano K, Ikeya T, Nakamura K, Takagi K, Fukuda K, Suzuki K, Fujita Y. Repeat endoscopic submucosal dissection for recurrent gastric cancers after endoscopic submucosal dissection. World J Gastrointest Endosc. 2013; 5(12):600-4.

Shimizu S, Tada M, Kawai K. Early gastric cancer: its surveillance and natural course. Endoscopy. 1995; 27(1):27-31.

Sibille A, Descamps C, Jonard P, Dive C, Warzeé P, Schapira M, Geubel A. Endoscopic Nd:YAG treatment of superficial gastric carcinoma experience in 18 Western inoperable patients. Gastrointest Endosc. 1995; 42(4):340-5.

Siegel R, Naishadham D, Jemal A. Cancer statistics for Hispanics/Latinos, 2012. CA Cancer J Clin. 2012; 62(5):283-98.

Siegel RL, Miller KD, Jemal A. Cancer statistics, 2015. CA Cancer J Clin. $2015 ; 65(1): 5-29$. 
SIGN - Scottish Intercollegiate Guidelines Network, Healthcare Improvement Scotland, Edinburgh. Disponível em: <http://www.sign.ac.uk/methodology/ checklists.html>. Acesso em: 24 mai. 2014.

Soetikno RM, Gotoda T, Nakanishi Y, Soehendra N. Endoscopic mucosal resection. Gastrointest Endosc. 2003; 57(4): 567-79.

Song F, Parekh S, Hooper L, Loke YK, Ryder J, Sutton AJ, Hing C, Kwok CS, Pang C, Harvey I. Dissemination and publication of research findings: an updated review of related biases. Health Technol Assess. 2010; 14(8):iii, ixxi, 1-193.

Sterne JAC, Sutton AJ, Ioannidis JPA, Terrin N, Jones DR, Lau J, Carpenter J, Rücker G, Harbord RM, Schmid CH, Tetzlaff J, Deeks JJ, Peters J, Macaskill P, Schwarzer G, Duval S, Altman DG, Moher D, Higgins JPT. Recommendations for examining and interpreting funnel plot asymmetry in meta-analyses of randomised controlled trials. BMJ. 2011; 342:d4002.

Subramanian V, Ragunath $\mathrm{K}$. Advanced endoscopic imaging: a review of commercially available technologies. Clin Gastroenterol Hepatol. 2014; 12(3):368-76.e1.

Tada K, Oda I, Yokoi C, Taniguchi T, Sakamoto T, Suzuki H, Nonaka S, Yoshinaga S, Saito Y, Gotoda T. Pilot study on clinical effectiveness of autofluorescence imaging for early gastric cancer diagnosis by less experienced endoscopists. Diagn Ther Endosc. 2011; 2011:419136. 
Teixeira CR, Haruma K, Teshima H, Shimamoto T, Tanaka S, Yamamoto G, Sumii K, Kajiyama G. Comparative report on endoscopic and surgical treatment for early gastric cancer in elderly patients. Arq Gastroenterol. 1992; 29(3):75-9.

The general rules for the gastric cancer study in surgery. Jpn J Surg. 1973; 3(1):61-71.

The Paris endoscopic classification of superficial neoplastic lesions: esophagus, stomach, and colon: November 30 to December 1, 2002. Gastrointest Endosc. 2003; 58(6 Suppl):S3-43.

Tokioka S, Umegaki E, Murano M, Takeuchi N, Takeuchi T, Kawakami K, Yoda Y, Kojima Y, Higuchi K. Utility and problems of endoscopic submucosal dissection for early gastric cancer in elderly patients. J Gastroenterol Hepatol. 2012; 27(Suppl 3):63-9.

Tomita T, Arai E, Kohno T, Kondo T, Kim Y, Oshima T, Hori K, Watari J, Matsumoto $\mathrm{T}$, Miwa $\mathrm{H}$. Outcomes of treatment of argon plasma coagulation therapy in elderly or high-risk patients with early gastric cancer: a comparison of outcomes among experienced and nonexperienced endoscopists. J Clin Gastroenterol. 2011; 45(6):e54-9.

Toyokawa T, Fujita I, Morikawa T, Okamoto A, Miyasaka R, Watanabe K, Horii J, Gobaru M, Terao M, Murakami T, Tomoda J. Clinical outcomes of ESD for early gastric neoplasms in elderly patients. Eur J Clin Invest. 2011; 41(5):474-8. 
Tsendsuren T, Jun SM, Mian XH. Usefulness of endoscopic ultrasonography in preoperative TNM staging of gastric cancer. World J Gastroenterol. 2006; 12(1):43-7.

Wanebo HJ, Kennedy BJ, Chmiel J, Steele G Jr, Winchester D, Osteen R. Cancer of the stomach. A patient care study by the American College of Surgeons. Ann Surg. 1993; 218(5):583-92.

Wang W, Zhang X, Shen C, Zhi X, Wang B, Xu Z. Laparoscopic versus open total gastrectomy for gastric cancer: an updated meta-analysis. PLoS One. 2014; 9(2): e88753.

Wroblewski LE, Peek RM Jr, Wilson KT. Helicobacter pylori and gastric cancer: factors that modulate disease risk. Clin Microbiol Rev. 2010; 23(4):713-39.

Yamaguchi N, Isomoto H, Fukuda E, Ikeda K, Nishiyama H, Akiyama M, Ozawa E, Ohnita K, Hayashi T, Nakao K, Kohno S, Shikuwa S. Clinical outcomes of endoscopic submucosal dissection for early gastric cancer by indication criteria. Digestion. 2009; 80(3):173-81.

Yamamoto $\mathrm{H}$, Kita $\mathrm{H}$. Endoscopic therapy of early gastric cancer. Best Pract Res Clin Gastroenterol. 2005; 19(6):909-26.

Yanai H, Fujimura H, Suzumi M, Matsuura S, Awaya N, Noguchi T, Karita M, Tada M, Okita K, Aibe T. Delineation of the gastric muscularis mucosae and assessment of depth of invasion of early gastric cancer using a 20-megahertz endoscopic ultrasound probe. Gastrointest Endosc. 1993; 39(4):505-12. 
Yanai H, Tada M, Karita M, Okita K. Diagnostic utility of 20-megahertz linear endoscopic ultrasonography in early gastric cancer. Gastrointest Endosc. 1996; 44(1):29-33.

Yang HK, Suh YS, Lee HJ. Minimally invasive approaches for gastric cancer - Korean experience. J Surg Oncol. 2013; 107(3): 277-81.

Yantiss RK, Odze RD. Optimal approach to obtaining mucosal biopsies for assessment of inflammatory disorders of the gastrointestinal tract. $A m \mathrm{~J}$ Gastroenterol. 2009; 104(3):774-83.

Yao K, Doyama H, Gotoda T, Ishikawa H, Nagahama T, Yokoi C, Oda I, Machida H, Uchita K, Tabuchi M. Diagnostic performance and limitations of magnifying narrow-band imaging in screening endoscopy of early gastric cancer: a prospective multicenter feasibility study. Gastric Cancer. 2014; 17(4):669-79.

Yasuda K, Mizuma Y, Nakajima M, Kawai K. Endoscopic laser treatment for early gastric cancer. Endoscopy. 1993; 25(7):451-4.

Yoshida S, Tanaka S, Kunihiro K, Mitsuoka Y, Hara M, Kitadai Y, Hata J, Yoshihara M, Haruma K, Hayakawa N, Chayama K. Diagnostic ability of high-frequency ultrasound probe sonography in staging early gastric cancer, specially for submucosal invasion. Abdom Imaging. 2005; 30(5):518-23.

Zilberstein B, Brücher BLDM, Coimbra BGM, Jacob CE, Barchi LC, Joaquim $\mathrm{H}$, Cecconello I. Gastric cancer: aspects of minimal invasive technique. $\mathrm{J}$ Gastroint Dig Syst. 2013a; 3(5):158. 
Zilberstein B, Costa Martins B, Jacob CE, Bresciani CJC, Lopasso FP, Cleva R, Pinto Júnior PE, Ribeiro-Júnior $U$, Perez RO, Gama-Rodrigues J. Complications of gastrectomy with lymphadenectomy in gastric cancer. Gastric Cancer. 2004; 7(4): 254-9.

Zilberstein B, Jacob CE, Cecconello I. Gastric cancer trends in epidemiology. Arq Gastroenterol. 2012; 49(3):177-8.

Zilberstein B, Malheiros C, Lourenço LG, Kassab P, Jacob CE, Weston AC, Bresciani CJ, Castro O, Gama-Rodrigues J; Grupo do Consenso, Borin AA, Buchpiegel C, Montagnini A, Leite CV, Deutsch CR, Kruel CD, Mucerino D, Wohnrath D, Ilias E, Mrué F, Maluf-Filho F, Rocha F, de Souza F, Tomasich FS, Ishak G, Laporte G, de Souza HP, Cecconello I, Eisig J, Ohana J, Sabagga J, del Grande JC, de Jesus JP, Soares J, Dias LA, Moreira LF, Correa M, Carvalho M, Andreollo NA, Áquila ND, Czeczko NG, Kruel N, Forones NM, da Motta OM, Malafaia O, Assumpção P, Leonardi P, Sakai P, Rocha PR, Colleoni R, Gurgel R, Coral RP, Chalub S, Ribeiro- Junior U, Alves VA, Vasquez Vde L, Nadalin V; Brazilian Association of Gastric Cancer. Brazilian consensus in gastric cancer: guidelines for gastric cancer in Brazil. Arq Bras Cir Dig. 2013b; 26(1):2-6. 
APÊNDICES 


\title{
Apêndice A - Classificação TNM do câncer de estômago - $7^{\text {a }}$ edição - American Joint Committee on Cancer (AJCC) ${ }^{6}$
}

\author{
Tumor primário (T)
}

TX - tumor primário não pode ser avaliado

TO - sem evidência de tumor primário

Tis - carcinoma in situ - tumor intraepitelial sem invasão da lâmina própria

T1 - tumor invade a lâmina própria, muscularis mucosae ou submucosa

T1a - tumor invade a lâmina própria ou muscularis mucosae

T1b - tumor invade a submucosa

T2 - tumor invade a muscular própria*

T3 - tumor invade o tecido conjuntivo subseroso sem comprometer o peritônio visceral

T4 - tumor invade a serosa (peritônio visceral) ou estruturas adjacentes $\Uparrow \Delta$

T4a - tumor invade a serosa (peritônio visceral)

T4b - tumor invade estruturas adjacentes

\section{Linfonodos regionais $(\mathrm{N})$}

$N X$ - linfonodos regionais não podem ser avaliados

NO - ausência de metástases linfonodais regionais\#

N1 - presença de metástases em 1-2 linfonodos regionais

N2 - presença de metástases em 3-6 linfonodos regionais

N3 - presença de metástases em 7 ou mais linfonodos regionais

N3a - presença de metástases em 7-15 linfonodos regionais

$\mathrm{N} 3 \mathrm{~b}$ - presença de metástases em 16 ou mais linfonodos regionais

\section{Metástases à distância (M)}

MO - ausência de metástases à distância

M1 - presença de metástases à distância

* Um tumor pode penetrar na camada muscular própria com extensão para o ligamento gastrocólico ou gastro-hepático, ou para o omento maior ou menor, sem perfuração do peritônio visceral que recobre estas estruturas. Nestes casos, o tumor é classificado como T3. Se existe perfuração do peritônio visceral que recobre os ligamentos ou os omentos, o tumor deve ser classificado como T4.

I As estruturas adjacentes do estômago incluem: baço, cólon transverso, fígado, diafragma, pâncreas, parede abdominal, glândula adrenal, rim, intestino delgado e retroperitônio.

$\Delta$ A extensão intramural para o duodeno ou esôfago é classificada pela profundidade da maior invasão em quaisquer um destes locais, incluindo o estômago.

\# A designação de pNO deve ser utilizada se todos os linfonodos examinados forem negativos, independentemente do número de linfonodos removidos e analisados.

6 São consideradas câncer gástrico precoce as lesões destacadas em cinza. 


\section{Apêndice B - Classificação de Vienna das neoplasias epiteliais gastrointestinais}

A Classificação de Vienna considera as seguintes categorias de lesões:

1. Categoria 1 - negativo para neoplasia/displasia

2. Categoria 2 - indefinido para neoplasia/displasia

3. Categoria 3 - neoplasia não-invasiva de baixo grau (adenoma ou displasia de baixo grau)

4. Categoria 4 - neoplasia não-invasiva de alto grau

4.1. adenoma ou displasia de alto grau

4.2. carcinoma não-invasivo (carcinoma in situ)

4.3. suspeita de carcinoma invasivo

5. Categoria 5 - neoplasia invasiva

5.1. carcinoma intramucoso (invasão até a lâmina própria ou muscularis mucosae)

5.2. carcinoma submucoso ou mais avançado 


\section{Apêndice C - Classificação Japonesa Macroscópica dos Carcinomas} Superficiais Gástricos (Japanese Classification of Gastric Carcinoma)

O sistema reconhece quatro tipos de cânceres precoces endoluminais:

1. Tipo I - lesões polipoides ou protuberantes

Ip - pedunculada

Isp - subpedunculada

Is - séssil

2. Tipo II - lesões planas

Ila - levemente elevada

Ilb - plana

IIc - levemente deprimida

3. Tipo III - lesões ulceradas

4. Tipo IV - lesões com crescimento lateral (lateral spreading) 


\section{Apêndice D - MeSH e seus sinônimos remissivos}

Medical Subject Headings (MeSH) é um sistema (descritor) de metadados médicos, em língua inglesa, que contempla a nomenclatura utilizada na indexação de artigos (palavraschave) na literatura e mantida pela U.S. National Library of Medicine. O MeSH utiliza-se do sistema Medline/ PubMed e, basicamente, fornece dados que facilitam o entendimento dos relacionamentos e a utilidade das informações dos dados (Medical Subject Headings, 2015).

Este apêndice, fornece uma visão detalhada dos termos MeSH utilizados na estratégia de busca mencionada no corpo do trabalho. Desta forma, são relatados, aqui, todos os entry terms, ou seja, os termos sinônimos que remetem ao termo $\mathrm{MeSH}$ anteriormente descrito, mantendo-se a estrutura básica da busca nos componentes População (P), Intervenção (I) e Comparação (C).

P: “(((Neoplasm, Stomach OR Stomach Neoplasm OR Neoplasms, Stomach OR Gastric Neoplasms OR Gastric Neoplasm OR Neoplasm, Gastric OR Neoplasms, Gastric OR Cancer of Stomach OR Stomach Cancers OR Gastric Cancer OR Cancer, Gastric OR Cancers, Gastric OR Gastric Cancers OR Stomach Cancer OR Cancer, Stomach OR Cancers, Stomach OR Cancer of the Stomach OR Gastric Cancer, Familial Diffuse) OR ((Adenocarcinoma OR Carcinoma OR Early Detection of Cancer OR Carcinoma in Situ OR Polyps) AND (Stomach OR Gastric)) OR ((Gastric Mucosa OR Early Diagnosis) AND (Gastric Cancer))) AND

I: (Endoscopy OR Surgical Procedures, Endoscopic OR Procedure, Endoscopic Surgical OR Procedures, Endoscopic Surgical OR Surgical Procedure, Endoscopic OR Endoscopy, Surgical OR Surgical Endoscopy OR Endoscopic Surgical Procedure OR Endoscopic Surgical Procedures OR Gastroscopy OR Gastroscopies OR Gastroscopic Surgical Procedures OR Gastroscopic Surgical Procedure OR Procedure, Gastroscopic Surgical OR Procedures, Gastroscopic Surgical OR Surgical Procedure, Gastroscopic OR Surgery, Gastroscopic OR Surgical Procedures, Gastroscopic OR Gastroscopic Surgery OR Gastroscopic Surgeries OR Surgeries, Gastroscopic OR Mucosectomy OR Polypectomy OR ESD OR Endoscopic Submucosal Dissection OR EMR OR Endoscopic Mucosal Resection OR Endoscopies, Gastrointestinal OR Gastrointestinal Endoscopies OR Gastrointestinal Endoscopy OR Surgical Procedures, Endoscopic Gastrointestinal OR Endoscopic Gastrointestinal Surgical Procedures OR Gastrointestinal Endoscopic Surgical Procedures OR Surgical Procedure, Endoscopic Gastrointestinal OR Procedure, Gastrointestinal Endoscopic Surgical OR Procedures, Endoscopic Gastrointestinal Surgical OR Procedures, Gastrointestinal Endoscopic Surgical OR Endoscopic Gastrointestinal Surgery OR Endoscopic Gastrointestinal Surgeries OR Gastrointestinal Surgeries, Endoscopic OR Gastrointestinal Surgery, Endoscopic OR Surgeries, Endoscopic Gastrointestinal OR Surgery, Endoscopic Gastrointestinal Procedure OR Endoscopic Gastrointestinal, Surgical OR Endoscopy, Digestive System OR Endoscopy, Gastrointestinal) AND. 
C: (Gastrectomy OR Gastrectomies OR Procedure, Digestive System Surgical OR Surgical Procedure, Digestive System OR Surgical Procedures, Digestive System OR Digestive System Surgical Procedure OR Procedures, Digestive System Surgical OR Gastrointestinal Surgical Procedure OR Gastrointestinal Surgical Procedures OR Surgical Procedure, Gastrointestinal OR Surgical Procedures, Gastrointestinal OR Procedure, Gastrointestinal Surgical OR Procedures, Gastrointestinal Surgical OR Surgery OR General Surgery OR Minimal Surgical Procedure OR Minimal Surgical Procedures OR Minimally Invasive Surgery OR Minimally Invasive Surgeries OR Surgeries, Minimally Invasive OR Surgery, Minimally Invasive OR Minimally Invasive Surgical Procedures OR Procedure, Minimal Surgical OR Procedures, Minimal Surgical OR Procedures, Minimally Invasive Surgical OR Surgical Procedure, Minimal OR Surgical Procedures, Minimal OR Surgical Procedures, Minimal Access OR Minimal Access Surgical Procedures OR Procedures, Minimal Access Surgical))".

Fonte: Medical Subject Headings. Bethesda: U.S. National Library of Medicine. Disponível em: <https://www.nlm.nih.gov/mesh/>. Acesso em 15 dez. 2015. 